\title{
EFFECTS OF SOFT INTERACTION AND NON-ISOTHERMAL BOUNDARY UPON LONG-TIME DYNAMICS OF RAREFIED GAS
}

\author{
RENJUN DUAN, FEIMIN HUANG, YONG WANG, AND ZHU ZHANG
}

\begin{abstract}
In the paper, assuming that the motion of rarefied gases in a bounded domain is governed by the angular cutoff Boltzmann equation with diffuse reflection boundary, we study the effects of both soft intermolecular interaction and non-isothermal wall temperature upon the long-time dynamics of solutions to the corresponding initial boundary value problem. Specifically, we are devoted to proving the existence and dynamical stability of stationary solutions whenever the boundary temperature has suitably small variations around a positive constant. For the proof of existence, we introduce a new mild formulation of solutions to the steady boundaryvalue problem along the speeded backward bicharacteristic, and develop the uniform estimates on approximate solutions in both $L^{2}$ and $L^{\infty}$. Such mild formulation proves to be useful for treating the steady problem with soft potentials even over unbounded domains. In showing the dynamical stability, a new point is that we can obtain the sub-exponential time-decay rate in $L^{\infty}$ without losing any velocity weight, which is actually quite different from the classical results as in 11,43 for the torus domain and essentially due to the diffuse reflection boundary and the boundedness of the domain.
\end{abstract}

\section{Contents}

1. Introduction

2. Preliminaries

3. Steady problem

4. Dynamical stability under small perturbations 34

5. Dynamical stability under a class of large perturbations 48

6. Appendix 59

References 63

\section{INTRODUCTION}

1.1. Boltzmann equation. Let a rarefied gas be contained in a bounded domain $\Omega$ in $\mathbb{R}^{3}$, and let $F=F(t, x, v)$ denote the density distribution function of gas particles with position $x \in \Omega$ and velocity $v \in \mathbb{R}^{3}$ at time $t>0$. We assume that $F$ is governed by the Boltzmann equation

$$
\partial_{t} F+v \cdot \nabla_{x} F=Q(F, F) .
$$

The Boltzmann collision term on the right-hand takes the non-symmetric bilinear form of

$$
Q\left(F_{1}, F_{2}\right)=\int_{\mathbb{R}^{3}} \int_{\mathbb{S}^{2}} B(|v-u|, \omega)\left[F_{1}\left(u^{\prime}\right) F_{2}\left(v^{\prime}\right)-F_{1}(u) F_{2}(v)\right] \mathrm{d} \omega \mathrm{d} u,
$$

Date: July 17, 2018.

2010 Mathematics Subject Classification. 35Q20, 35B20, 35B35, 35B45.

Key words and phrases. Botlzmann equation, soft potentials, bounded domain, non-isothermal boundary, diffuse reflection, steady problem, dynamical stability, large-amplitude initial data, a priori estimate. 
where the velocity pair $\left(v^{\prime}, u^{\prime}\right)$ is defined by the velocity pair $(v, u)$ as well as the parameter $\omega \in \mathbb{S}^{2}$ in terms of the relation

$$
v^{\prime}=v-[(v-u) \cdot \omega] \omega, \quad u^{\prime}=u+[(v-u) \cdot \omega] \omega,
$$

according to conservation laws of momentum and energy

$$
v^{\prime}+u^{\prime}=v+u, \quad\left|v^{\prime}\right|^{2}+\left|u^{\prime}\right|^{2}=|v|^{2}+|u|^{2},
$$

due to the elastic collision of two particles. To the end the Boltzmann collision kernel $B(|v-u|, \omega)$, depending only on the relative velocity $|v-u|$ and $\cos \phi=\omega \cdot(v-u) /|v-u|$, is assumed to satisfy

$$
B(|v-u|, \omega)=|v-u|^{\kappa} b(\phi),
$$

with

$$
-3<\kappa<0, \quad 0 \leq b(\phi) \leq C|\cos \phi|,
$$

for a generic constant $C>0$, namely, we consider in this paper the full range of soft potentials under the Grad's angular cutoff assumption.

1.2. Diffuse reflection boundary condition. We assume that $\Omega=\{\xi(x)<0\}$ is connected and bounded with $\xi(x)$ being a smooth function in $\mathbb{R}^{3}$. At each boundary point with $\xi(x)=$ 0 , we assume that $\nabla \xi(x) \neq 0$. The outward unit normal vector is therefore given by $n(x)=$ $\nabla \xi(x) /|\nabla \xi(x)|$. We define that $\Omega$ is strictly convex if there is $c_{\xi}>0$ such that $\sum_{i j} \partial_{i j} \xi(x) \eta^{i} \eta^{j} \geq$ $c_{\xi}|\eta|^{2}$ for all $x \in \bar{\Omega}$ and all $\eta \in \mathbb{R}^{3}$.

We denote the phase boundary of the phase space $\Omega \times \mathbb{R}^{3}$ as $\gamma=\partial \Omega \times \mathbb{R}^{3}$, and split $\gamma$ into three disjoint parts, outgoing boundary $\gamma_{+}$, the incoming boundary $\gamma_{-}$, and the singular boundary $\gamma_{0}$ for grazing velocities:

$$
\begin{aligned}
& \gamma_{+}=\left\{(x, v) \in \partial \Omega \times \mathbb{R}^{3}: n(x) \cdot v>0\right\}, \\
& \gamma_{-}=\left\{(x, v) \in \partial \Omega \times \mathbb{R}^{3}: n(x) \cdot v<0\right\}, \\
& \gamma_{0}=\left\{(x, v) \in \partial \Omega \times \mathbb{R}^{3}: n(x) \cdot v=0\right\} .
\end{aligned}
$$

We supplement the Boltzmann equation (1.1) with the diffuse reflection boundary condition

$$
\left.F(t, x, v)\right|_{\gamma_{-}}=\mu_{\theta}(v) \int_{v^{\prime} \cdot n(x)>0} F\left(t, x, v^{\prime}\right)\left\{v^{\prime} \cdot n(x)\right\} \mathrm{d} v^{\prime},
$$

where $\mu_{\theta}(v)$ is a local Maxwellian with a non-isothermal wall temperature $\theta=\theta(x)>0$ :

$$
\mu_{\theta}(v)=\frac{1}{2 \pi \theta^{2}(x)} e^{-\frac{|v|^{2}}{2 \theta(x)}} .
$$

Throughout this paper, we assume that $\theta(x)$ has a small variation around a fixed postive temperature $\theta_{0}>0$. Without loss of generality, we assume $\theta_{0}=1$, and for brevity we denote the global Maxwellian

$$
\mu=\mu(v) \equiv \mu_{\theta_{0}}(v)=\frac{1}{2 \pi} e^{-\frac{|v|^{2}}{2}} .
$$

1.3. Main results. Note that

$$
\int_{v \cdot n(x)>0} \mu_{\theta}(v)\{v \cdot n(x)\} \mathrm{d} v=1,
$$

for any $x \in \partial \Omega$, and hence $\mu_{\theta}(v)$ satisfies the boundary condition (1.5). However, it is straightforward to see that the stationary local Maxwellian $\mu_{\theta}(v)$ does not satisfy the Boltzmann equation (1.1) because of spatial variation unless $\theta(x)$ is constant on $\partial \Omega$. One may expect that the longtime behavior of solutions to (1.1) and (1.5) could be determined by the time-independent steady equation with the same boundary condition. Thus the study of this paper includes two parts. In the first part we investigate the steady problem in order to obtain the existence of stationary solutions, and in the second part we are devoted to showing the dynamical stability of the obtained 
stationary solutions under small perturbations and further under a class of large perturbations in velocity weighted $L^{\infty}$ spaces.

In what follows we present the main results of this paper. The first one is to clarify the well-posedness of the boundary-value problem on the Boltzmann equation with diffuse reflection boundary condition

$$
\left\{\begin{array}{l}
v \cdot \nabla_{x} F=Q(F, F), \quad(x, v) \in \Omega \times \mathbb{R}^{3}, \\
\left.F(x, v)\right|_{\gamma_{-}}=\mu_{\theta}(v) \int_{v^{\prime} \cdot n(x)>0} F\left(x, v^{\prime}\right)\left\{v^{\prime} \cdot n(x)\right\} \mathrm{d} v^{\prime} .
\end{array}\right.
$$

We define a velocity weight function

$$
w=w(v):=\left(1+|v|^{2}\right)^{\frac{\beta}{2}} e^{\varpi|v|^{\zeta}},
$$

where $\beta>0$ and $0<\zeta \leq 2$ are given constants, and $(\varpi, \zeta)$ belongs to

$$
\left\{\zeta=2,0<\varpi<\frac{1}{8}\right\} \cup\{0<\zeta<2, \varpi>0\} .
$$

Here and in the sequel, for brevity we have omitted the explicit dependence of $w$ on all parameters $\beta, \varpi$ and $\zeta$.

Theorem 1.1. Let $-3<\kappa<0, \beta>3+|\kappa|$, and $(\varpi, \zeta)$ belong to (1.10). For given $M>0$, there exist $\delta_{0}>0$ and $C>0$ such that if

$$
\delta:=\left|\theta-\theta_{0}\right|_{L \infty(\partial \Omega)} \leq \delta_{0},
$$

then there exists a unique nonnegative solution $F_{*}(x, v)=M \mu(v)+\mu^{\frac{1}{2}}(v) f_{*}(x, v) \geq 0$ to the steady problem (1.8), satisfying the mass conservation

$$
\int_{\Omega} \int_{\mathbb{R}^{3}} f_{*}(x, v) \mu^{\frac{1}{2}}(v) \mathrm{d} v \mathrm{~d} x=0,
$$

and the estimate

$$
\left\|w f_{*}\right\|_{L^{\infty}}+\left|w f_{*}\right|_{L^{\infty}(\gamma)} \leq C \delta .
$$

Moreover, if $\Omega$ is strictly convex and $\theta(x)$ is continuous on $\partial \Omega$, then $F_{*}$ is continuous on $(x, v) \in$ $\bar{\Omega} \times \mathbb{R}^{3} \backslash \gamma_{0}$.

For simplicity, through the paper we would take $M=1$ in Theorem 1.1 without loss of generality, namely, the stationary solution $F_{*}(x, v)$ has the same total mass as $\mu(v)$ in $\Omega$. Note that when there is no spatial variation on the boundary temperature, i.e., $\delta=0$, the stationary solution is reduced to the global Maxwellian.

The second result is concerned with the dynamical stability of $F_{*}(x, v)$ under small perturbations in $L^{\infty}$. We assume that (1.1) is also supplemented with initial data

$$
\left.F(t, x, v)\right|_{t=0}=F_{0}(x, v) .
$$

The goal is to show the large-time convergence of solutions of the initial-boundary value problem (1.1), (1.5) and (1.13) to the stationary solution $F_{*}(x, v)$, whenever they are sufficiently close to each other in some sense at initial time.

Theorem 1.2. Let $-3<\kappa<0, \beta>3+|\kappa|$ and $(\varpi, \zeta)$ belong to (1.10). Assume (1.11) with $\delta_{0}>0$ chosen to be further small enough. There exist constants $\varepsilon_{0}>0, C_{0}>0$ and $\lambda_{0}>0$ such that if $F_{0}(x, v)=F_{*}(x, v)+\mu^{\frac{1}{2}}(v) f_{0}(x, v) \geq 0$ satisfies the mass conservation

$$
\int_{\Omega} \int_{\mathbb{R}^{3}} f_{0}(x, v) \mu^{\frac{1}{2}}(v) \mathrm{d} v \mathrm{~d} x=0
$$

and

$$
\left\|w f_{0}\right\|_{L^{\infty}} \leq \varepsilon_{0}
$$


then the initial-boundary value problem (1.1), (1.5) and (1.13) on the Boltzmann equation admits a unique solution $F(t, x, v)=F_{*}(x, v)+\mu^{\frac{1}{2}}(v) f(t, x, v) \geq 0$ satisfying

$$
\int_{\Omega} \int_{\mathbb{R}^{3}} f(t, x, v) \mu^{\frac{1}{2}}(v) \mathrm{d} v \mathrm{~d} x=0
$$

and

$$
\|w f(t)\|_{L^{\infty}}+|w f(t)|_{L^{\infty}(\gamma)} \leq C_{0} e^{-\lambda_{0} t^{\alpha}}\left\|w f_{0}\right\|_{L^{\infty}}
$$

for all $t \geq 0$, where $\alpha \in(0,1)$ is given by

$$
\alpha:=\frac{\zeta}{\zeta+|\kappa|} .
$$

Moreover, if $\Omega$ is strictly convex, $F_{0}(x, v)$ is continuous except on $\gamma_{0}$ and satisfying

$$
\left.F_{0}(x, v)\right|_{\gamma_{-}}=\mu_{\theta}(v) \int_{v^{\prime} \cdot n(x)>0} F_{0}\left(x, v^{\prime}\right)\left\{v^{\prime} \cdot n(x)\right\} \mathrm{d} v^{\prime},
$$

and $\theta(x)$ is continuous on $\partial \Omega$, then $F(t, x, v)$ is continuous in $[0, \infty) \times\left\{\bar{\Omega} \times \mathbb{R}^{3} \backslash \gamma_{0}\right\}$.

We remark that the value of $\alpha$ in (1.18), which is optimal in terms of the exponential velocity weighted function space, can be formally determined as in [11; we will come back to this point later. By (1.17), we have obtained the global existence and large-time behavior of solutions simultaneously in the velocity-weighted $L^{\infty}$ space which is the same as that initial data belong to.

One may notice from (1.15) in Theorem 1.2 above that the initial perturbation $f_{0}(x, v)$ is required to generally have a small amplitude in the velocity-weighted $L^{\infty}$ space. The goal of the third result is to relax such restriction by allowing $F_{0}(x, v)$ to have large oscillations around the stationary solution $F_{*}(x, v)$ with the price that the initial perturbation $f_{0}(x, v)$ is small enough in some $L^{p}$ norm for $1<p<\infty$.

Theorem 1.3. Assume that all conditions in Theorem 1.2 are satisfied, and additionally let

$$
\max \left\{\frac{3}{2}, \frac{3}{3+\kappa}\right\}<p<\infty, \quad \beta>\max \{3+|\kappa|, 4\} .
$$

Assume (1.11) with $\delta_{0}>0$ chosen to be further small enough and initial data $F_{0}(x, v)=F_{*}(x, v)+$ $\mu^{\frac{1}{2}}(v) f_{0}(x, v) \geq 0$ satisfies the mass conservation (1.14). There exist constants $\varepsilon_{1}>0, C_{1}>1$ and $C_{2}>1$ such that if $f_{0}(x, v)$ satisfies

$$
M_{0}:=\left\|w f_{0}\right\|_{L^{\infty}} \leq C_{1}|\log \delta|
$$

and

$$
\left\|f_{0}\right\|_{L^{p}} \leq \varepsilon_{1},
$$

then the initial-boundary value problem (1.1), (1.5) and (1.13) on the Boltzmann equation admits a unique solution $F(t, x, v)=F_{*}(x, v)+\mu^{\frac{1}{2}}(v) f(t, x, v) \geq 0$ satisfying (1.16) and

$$
\|w f(t)\|_{L^{\infty}}+|w f(t)|_{L^{\infty}(\gamma)} \leq C_{2} e^{C_{2} M_{0}} e^{-\lambda_{0} t^{\alpha}}\left\|w f_{0}\right\|_{L^{\infty}},
$$

for all $t \geq 0$, where $\alpha$ is the same as in (1.18) and $\lambda_{0}$ is the same as in (1.17). Moreover, if $\Omega$ is strictly convex, $F_{0}(x, v)$ is continuous except on $\gamma_{0}$ satisfying (1.19), and $\theta(x)$ is continuous over $\partial \Omega$, then $F(t, x, v)$ is continuous in $[0, \infty) \times\left\{\bar{\Omega} \times \mathbb{R}^{3} \backslash \gamma_{0}\right\}$.

Remark 1.4. We give a few remarks in order on the above theorem.

(a) Note that $C_{1}$ is independent of $\delta$. Then, from (1.21), $M_{0}=\left\|w f_{0}\right\|_{L^{\infty}}$ can be arbitrarily large, provided that both $\delta$ and $\left\|f_{0}\right\|_{L^{p}}$ are sufficiently small. Particularly, if one takes $\delta=0$ corresponding to the isothermal boundary temperature, there is no restriction on the upper bound of $M_{0}$. However, it is unclear how to remove the condition (1.21) whenever $\delta>0$. 
(b) From (1.20), $p$ has to be large enough as $\kappa$ gets close to -3 . The condition (1.22) for the smallness of $f_{0}$ in $L^{p}$ is different from that in [18] and [19] where $L^{1}$ norm and $L^{2}$ norm were used respectively. Note that (1.22) can be also guaranteed by the smallness of $L^{1}$ or $L^{2}$ norm of $f_{0}$ and the velocity-weighted $L^{\infty}$ bound with the help of the interpolation.

(c) As already mentioned for Theorem 1.2, by (1.23) we have obtained the global existence and large-time behavior of solutions simultaneously in the velocity-weighted $L^{\infty}$ space which is the same as that initial data belong to. Estimate (1.23) also implies that the solution may grow with an exponential rate of $M_{0}$ within a short time.

1.4. Comments and literature. The focus of this paper is on the effects of both the soft intermolecular interaction and the non-isothermal wall temperature on the large-time behaviour of solutions to the initial-boundary value problem on the Boltzmann equation. In what follows we review some known results related to our results and also give comments on how such effects occur.

(a) Effect of soft potentials. First of all, we discuss the effect of soft potentials on the global well-posedness of the Boltzmann equation in perturbation framework. Compared to the hard potentials, the main difficulty is the lack of the spectral gap of the linearized Boltzmann operator $L$, for instance, the multiplication operator $\nu(v) \sim\langle v\rangle^{\kappa}$ has no strictly positive lower bound over large velocities $|v|$ for $\kappa<0$.

In the spatially periodic domain $\mathbb{T}^{3}$, Caflish [10, 11] first constructed the global-in-time solution for $-1<\kappa<0$ and also studied the large-time behavior of solutions, where the proof is based on the time-decay property of the linearised equation together with the bootstrap argument on the nonlinear equation. One important observation by Caflish is that the function $\exp \left\{-\langle v\rangle^{\kappa} t-c|v|^{2}\right\}$, obtained as the solution to the spatially homogeneous equation $\partial_{t} f+\langle v\rangle^{\kappa} f=0$ with initial data $f(0, v)=\exp \left\{-c|v|^{2}\right\}$, decays in time with a rate $\exp \left\{-\lambda t^{\beta}\right\}$ with $\beta=2 /(2+|\kappa|)$ by taking the infimum of $\langle v\rangle^{\kappa} t+c|v|^{2}$ in $v \in \mathbb{R}^{3}$. We remark that such sub-exponential time-decay is ensured essentially by adding more exponential velocity weight at initial time; see [11, equations (3.1) and (3.2) of Theorem 3.1 on page 76].

Independently, Ukai-Asano [44] developed the semigroup theory in the case of soft potentials $-1<\kappa<0$, and also obtained the global solution as well as the large-time behavior of solutions for the problem in the whole space $\mathbb{R}^{3}$. As pointed out by [44, Theorem 9.1 and Remark 9.1 on page 96], no solutions have been found in the large in time if initial data and solutions belong to the function space with the same velocity weights. We remark that it is the same situation if one adopts the approach of 44 to treat the case of $\mathbb{T}^{3}$, for instance, one can obtain the arbitrarily large algebraic time-decay rate by postulating more polynomial velocity weights on initial data.

By the pure energy method in high-order Sobolev spaces, Guo [28 constructed the global solutions over $\mathbb{T}^{3}$ for the full range of soft potentials $-3<\kappa<0$, but the large-time behavior of solutions was left. This problem was later completely solved by Strain-Guo in [42] and [43] in terms of the same spirit as in [4] and [11] by putting additional polynomial or exponential velocity weights on initial data. Such approach was also applied by Strain [40] to study the asymptotic stability of the relativistic Boltzmann equation for the soft potentials in $\mathbb{T}^{3}$.

In the case of $\mathbb{R}^{3}$, we also mention Duan-Yang-Zhao [20] and Strain [41] to treat the optimal large-time behavior of solutions for $-3<\kappa<0$. Particularly, 20] found a velocity weight function containing an exponential factor $\exp \left\{c|v|^{2} /(1+t)^{q}\right\}$. We remark that this kind of weight could be useful for simultaneously dealing with the global existence and large-time behavior of solutions for the problem in the torus domain or even in the general bounded domain (for instance, [36]), since the typical function $\exp \left\{-\langle v\rangle^{\gamma} t-c|v|^{2} /(1+t)^{q}\right\}$ induces a time-decay rate $\exp \left\{-\lambda t^{\beta^{\prime}}\right\}$ with $\beta^{\prime}=(2-q|\kappa|) /(2+|\kappa|)$. Therefore, the large-time behavior of solutions is gained by making the velocity weight in the solution space become lower and lower as time goes on. Indeed, this is also in the same spirit as in [4] on the basis of the velocity-time splitting technique.

By comparison with those results mentioned above, Theorem 1.2 or 1.3 implies that the largetime behavior of solutions to the initial-boundary value problem under consideration of this paper 
is established in the situation where solutions and initial data enjoy the same exponential velocity weight. In other words, to obtain the sub-exponential time-decay for soft potentials, it is no need to put any additional velocity weight on initial data. Roughly speaking, the main reason to realize this point is due to not only the boundedness of the domain but also the diffuse reflection boundary condition, which will be explained in more detail later on. We remark that the results are nontrivial to obtain even if the wall temperature is reduced to a constant implying that the stationary solution $F_{*}(x, v)$ is a global Maxwellian.

(b) Effect of non-isothermal boundary. The non-isothermal wall temperature provides an inhomogeneous source to force the Boltzmann solution to tend in large time to nontrivial stationary profiles. We review related works in the following two aspects which also involve the case of isothermal boundary. We mainly focus on general bounded domains. There exist also a number of papers in the setting of one-dimensional bounded intervals with different types of boundary conditions, cf. [37, 39]. Among them, we point out that Arkeryd together with his collaborators have made great contributions to this direction, for instance, 3, 4, 7] and reference therein, where solutions are constructed mainly for large boundary data. The existence and dynamical stability of the stationary solution in a slab with diffuse reflection boundary was considered by $\mathrm{Yu} 46$ in terms of a new probabilistic approach. Hydrodynamic limit to the compressible Navier-Stokes equations for the stationary Boltzmann equation in a slab was studied by Esposito-Lebowitz-Marra [23, 24]. For other related works on the effects of non-isothermal boundary, we also mention [14, 34, 35].

- Time-dependent IBVP in general bounded domains. A first investigation of the IBVP was made by Hamdache 32 for a large-data existence theory in the sense of DiPerna-Lions [17. Extensions of such result have been made in [2, 5, 12, 38, in several directions including the case of general diffuse reflection with variable wall temperature. The large-time behavior of weak solutions was studied in [6, 15, 16]. In the perturbation framework, via the idea of [45, Guo [29] developed a new approach to treat the global existence, uniqueness and continuity of bounded solutions with different types of boundary conditions. Further progress on high-order Sobolev regularity of solutions was recently made in [30; see also references therein. For other related works on the study of the IBVP on the nonlinear Boltzmann equation, we would mention 9 for the general Maxwell boundary condition, 31] for the global existence of solutions with weakly inhomogeneous data in the case of specular reflection, 33 for the specular boundary condition in convex domains with $C^{3}$ smoothness, and [36] for a direct extension of [29] from hard potentials to soft potentials.

- Steady problem in general bounded domains. There are much less known results on the mathematical analysis of the stationary Boltzmann equation in a general 3D bounded domain. First of all, it seems still open to establish a large-data DiPerna-Lions existence theory in the steady case; see [8], however, for an $L^{1}$ existence theorem with inflow data when the collision operator is truncated for small velocities. In Guiraud [26, 27, existence of stationary solutions was proved in convex bounded domains, but the positivity of obtained solutions remained unclear. Via the approach in [29], Esposito et al 21] constructed the small-amplitude non-Maxwellian stationary solution for diffuse reflection when the space-dependent wall temperature has a small variation around a positive constant for hard potentials, and further obtained the positivity of stationary solutions as a consequence of the dynamical stability for the time-evolutionary Boltzmann equation. Indeed, 21] motivates us to study the steady Boltzmann equation for soft potentials, and we will explain the new mild formulation of solutions as well as new a priori estimates in more detail later on.

The hydrodynamic limit of the stationary Boltzmann equation on bounded domains in the incompressible setting was recently justified in 22. Notice that such research topic was also discussed in 1] where the authors have particularly shown the non-existence of steady solutions for the Boltzmann equation with smooth divergence-free external forces in bounded domains with specular reflection. 
1.5. Strategy of the proof. In what follows, we briefly explain the key points in our proof of Theorem 1.1. Theorem 1.2 and Theorem 1.3 respectively.

(a) First, for the proof of Theorem 1.1 the key step is to establish a priori $L^{\infty}$-estimates on the steady solutions. The major difficulty comes from the degeneracy of collision frequency $\nu(v) \rightarrow 0$ as $|v| \rightarrow \infty$. Our strategy of overcoming this relies on introducing a new mild formulation of the steady Boltzmann equation along a speeded backward bi-characteristics on which the particles with large velocity move much faster than one along the classical characteristics. Precisely, we need to consider the solvability of the linearized steady Boltzmann equation with inhomogeneous source and boundary data

$$
\left\{\begin{array}{l}
v \cdot \nabla_{x} f+L f=g \\
\left.f\right|_{\gamma_{-}}=P_{\gamma} f+r
\end{array}\right.
$$

See Lemma 3.8, particularly (3.72) for the $L^{\infty}$ bound of $f$ in terms of $g$ and $r$. Basing on Lemma 3.8. Theorem 1.1 follows by showing the convergence of the iterative approximate solution sequence.

To show Lemma 3.8, we turn to study in Lemma 3.5 the solvability of the following approximate boundary-value problem:

$$
\left\{\begin{array}{l}
\mathcal{L}_{\lambda} f:=\varepsilon f+v \cdot \nabla_{x} f+\nu(v) f-\lambda K f=g \\
\left.f\right|_{\gamma_{-}}=\left(1-\frac{1}{n}\right) P_{\gamma} f+r .
\end{array}\right.
$$

Here, compared to the previous works [21, 22, we input an extra parameter $\lambda \in[0,1]$ in order to carry out a new strategy of the construction of solutions by making the interplay of $L^{2}$ and $L^{\infty}$ estimates. Specifically, we divide the proof by several steps as follows:

Step 1. To show the well-posedness of $\mathcal{L}_{0}^{-1}$ for $\lambda=0$. The reason why we start from the case of $\lambda=0$ is that there is no linear collision term $K f$. In this case, we are able to directly construct the approximate solutions by solving the inflow problem, so the $L^{\infty}$ bound of approximate solutions is a consequence of $L^{\infty}$ bounds of the source term $g$ as well as the corresponding boundary data. The uniform bound of solutions can be obtained in the same way as in the next Step 2.

Step 2. To obtain the a priori estimates of solutions in both $L^{2}$ and $L^{\infty}$ uniform in all parameters $\varepsilon, n$ and $\lambda$. For the $L^{2}$ estimate, it is based on the fact that

$$
\nu-\lambda K=(1-\lambda) \nu+\lambda L
$$

with $0 \leq \lambda \leq 1$ is still nonnegative. For the velocity-weighted $L^{\infty}$ estimate on $h=w f$, we formally multiply the equation of (1.25) by $\left(1+|v|^{2}\right)^{|\kappa| / 2}$ so as to get

$$
\hat{v} \cdot \nabla_{x} h+\left(1+|v|^{2}\right)^{|\kappa| / 2}[\varepsilon+\nu(v)] h=\lambda\left(1+|v|^{2}\right)^{|\kappa| / 2} K_{w} h+\left(1+|v|^{2}\right)^{|\kappa| / 2} w g,
$$

and then write it as the mild form along the backward bi-characteristic $[x-\hat{v}(t-s), v]$, where $\hat{v}=\left(1+|v|^{2}\right)^{|\kappa| / 2} v$ is the transport velocity speeded up by comparison with the original velocity $v$. The advantage of such new mild formulation is that the corresponding new collision frequency

$$
\hat{\nu}(v)=\left(1+|v|^{2}\right)^{|\kappa| / 2}[\varepsilon+\nu(v)]
$$

has a uniform-in- $\varepsilon$ strictly positive lower bound independent of $v$. This is crucial for obtaining $L^{\infty}$-estimates for the steady problem. It should be pointed out that by using such new mild formulation, the $L^{\infty}$-estimates are also valid for the case that the domain is unbounded, so that our method in principle could be used to further study other physically important problems, such as exterior problems and shock wave theory.

Step 3. To prove the well-posedness of $\mathcal{L}_{\lambda}^{-1}$ for any $\lambda \in\left[0, \lambda_{*}\right]$ with a constant $\lambda_{*}>0$ small enough. The main idea of showing the existence of solutions is based on the fixed point argument for the solution operator

$$
\mathcal{L}_{\lambda}^{-1} f=\mathcal{L}_{0}^{-1}(\lambda K f+g) .
$$


Note that the contraction property is essentially the consequence of the fact that we are restricted to $\lambda>0$ small enough. Once the existence of solutions is established, we also have the uniform estimates in $L^{2}$ and $L^{\infty}$ obtained in Step 2.

Step 4. To prove the well-posedness of $\mathcal{L}_{\lambda_{*}+\lambda}^{-1}$ for any $\lambda \in\left[0, \lambda_{*}\right]$ small enough. Formally, we have

$$
\mathcal{L}_{\lambda_{*}+\lambda}^{-1}=\mathcal{L}_{\lambda_{*}}^{-1}(\lambda K f+g) .
$$

Therefore, we may make use of the same arguments as in Step 3 and also obtain the corresponding uniform estimates. In the end, by repeating such procedure we can establish the solvability of $\mathcal{L}_{\lambda}^{-1}$ in the case of $\lambda=1$, and complete the construction of approximate solutions to the original boundary-value problem (1.24).

(b) Secondly, for the proof of Theorem 1.2, the key step is to study the time-decay structure of linearized IBVP problem around the steady solution provided by Theorem 1.1, In general, it is hard to obtain a satisfactory decay due to the degeneracy of $\nu(v)$ at large velocity. Unfortunately, our new mild formulation above no longer works for the time-dependent problem. Some new thought should be involved in. As mentioned before, so far there are basically two ways to get the decay of the Boltzmann solution for soft potentials. The first one, which was developed by Guo and Strain [43, is to first establish the global existence of the solution with an extra sufficiently strong velocity weight and then obtain the decay of the solution without weight by an interpolation technique. Following their idea, Liu and Yang [36] extend their work into the IBVP problem. The other one, which is developed by Duan, Yang and Zhao [20, is to introduce a velocity weight involving a time dependent factor $\exp \left\{c|v|^{\zeta} /(1+t)^{q}\right\}$ in order to compensate the degeneracy of collision frequency. One can see that there is an extra restriction in both theories that the initial datum must involve additional velocity weight. One of main contributions in the present work is to remove such a restriction.

More precisely, we are able to obtain simultaneously the global existence and the sub-exponential decay of the solution, without loss of any weight. The key observation used in our arguments is twofold. The first is to split the large velocity part and small velocity part in the following type estimate:

$$
\int_{\max \left\{t-t_{\mathbf{b}}, 0\right\}}^{t} e^{-\nu(v)(t-s)} \mathrm{d} s=\int_{\max \left\{t-t_{\mathbf{b}}, 0\right\}}^{t} e^{-\nu(v)(t-s)}\left\{\mathbf{1}_{\left\{|v| \leq d_{\Omega}\right\}}+\mathbf{1}_{\left\{|v|>d_{\Omega}\right\}}\right\} \mathrm{d} s,
$$

so it holds that

$$
\int_{\max \left\{t-t_{\mathbf{b}}, 0\right\}}^{t} e^{-\nu(v)(t-s)} \mathrm{d} s \leq \int_{\max \left\{t-t_{\mathbf{b}}, 0\right\}}^{t} e^{-\bar{\nu}_{0}(t-s)} \mathbf{1}_{\left\{|v| \leq d_{\Omega}\right\}}+\mathbf{1}_{t-1 \leq s \leq t} \mathbf{1}_{\left\{|v|>d_{\Omega}\right\}} \mathrm{d} s,
$$

which is essentially based on the elementary fact that the backward exit time

$$
t_{\mathbf{b}}(x, v) \leq \frac{\operatorname{diam}(\Omega)}{|v|} .
$$

One can see that even for the soft potential, (1.26) still involves an exponential decay structure. The second is to notice that due to the diffusive reflection boundary condition, the boundary terms naturally exponentially decay in velocity. So, we can make use of the Caflish's idea to obtain that

$$
e^{-\nu(v)\left(t-t_{1}\right)} e^{-\frac{|v|^{2}}{16}} \leq C e^{-\lambda_{1}\left(t-t_{1}\right)^{\alpha}},
$$

where $\lambda_{1}>0$ is obtained by taking the infimum of $\nu(v)\left(t-t_{1}\right)+|v|^{2} / 16$ with respect to velocity. This reveals the decay structure for boundary terms. However, (1.26) and (1.27) only work when $\Omega$ is bounded and the solution satisfies the diffuse reflection boundary condition. So far we don't know how to deal with the same problem for the specular reflection or even in the torus. 
(c) Thirdly, the strategy of the proof of Theorem 1.3 is to use the linear decay theory provided by the second part to find a large time $T_{0}=T_{0}\left(M_{0}, \varepsilon_{0}\right)$, such that

$$
\left\|w f\left(T_{0}\right)\right\|_{L^{\infty}} \leq \varepsilon_{0} .
$$

Then we can extend our solution into $\left[T_{0}, \infty\right)$ by the previous small-amplitude theory. Since the initial data is allowed to have large oscillation around $F_{*}$, more efforts should be paid for treating the nonlinear term $w(v) \Gamma(f, f)$. The key point is to bound it pointwisely by a product of $L^{\infty}$-norm and $L^{p}$-norm with $\max \left\{\frac{3}{2}, \frac{3}{3+\kappa}\right\}<p<\infty$ and then apply a nonlinear iteration. To show (1.28), we have to require the following estimates holds:

$$
C\left(\sup _{0 \leq t \leq T_{0}}\|w f(t)\|_{L^{\infty}}\right)^{3} \cdot|\theta-1|_{L^{\infty}(\partial \Omega)} \lesssim \varepsilon_{0} .
$$

Hence the restriction (1.21) on the amplitude of initial data is naturally required.

1.6. Plan of the paper. In Section 2 we will provide some basic estimates on linear and nonlinear collision terms. In Section 3, we study the steady problem and give the proof of Theorem 1.1 for the existence of the stationary solution. In Section 4, we study the large-time asymptotic stability of the obtained stationary solution under small perturbations and give the proof of Theorem 1.2 . In Section 5, we further extend the result to the situation where initial perturbation can have large amplitude with an extra restriction but be small in $L^{p}$ norm, and give the proof of Theorem 1.3. In Appendix, we give the proof of a technical lemma which has been used before, and also give the proof of the local-in-time existence of solutions for completeness.

1.7. Notations. Throughout this paper, $C$ denotes a generic positive constant which may vary from line to line. $C_{a}, C_{b}, \cdots$ denote the generic positive constants depending on $a, b, \cdots$, respectively, which also may vary from line to line. $A \lesssim B$ means that there exists a constant $C>0$ such that $A \leq C B$. $\|\cdot\|_{L^{2}}$ denotes the standard $L^{2}\left(\Omega \times \mathbb{R}_{v}^{3}\right)$-norm and $\|\cdot\|_{L^{\infty}}$ denotes the $L^{\infty}\left(\Omega \times \mathbb{R}_{v}^{3}\right)$-norm. For the functions depending only in velocity $v$, we denote $\|\cdot\|_{L_{v}^{p}}$ as the $L^{p}\left(\mathbb{R}_{v}^{3}\right)$-norm and $\langle\cdot, \cdot\rangle$ as the $L^{2}\left(\Omega \times \mathbb{R}_{v}^{3}\right)$ inner product or $L^{2}\left(\mathbb{R}_{v}^{3}\right)$ inner product. Moreover, we denote $\|\cdot\|_{\nu}:=\|\sqrt{\nu} \cdot\|_{L^{2}}$. For the phase boundary integration, we define $d \gamma \equiv|n(x) \cdot v| d S(x) d x$, where $d S(x)$ is the surface measure and define $|f|_{L^{p}}^{p}=\int_{\gamma}|f(x, v)|^{p} d \gamma$ and the corresponding space is denoted as $L^{p}\left(\partial \Omega \times \mathbb{R}^{3}\right)=L^{p}\left(\partial \Omega \times \mathbb{R}^{3} ; d \gamma\right)$. Furthermore, we denote $|f|_{L^{p}\left(\gamma_{ \pm}\right)}=\left|f I_{\gamma_{ \pm}}\right|_{L^{p}}$ and $|f|_{L^{\infty}\left(\gamma_{ \pm}\right)}=\left|f I_{\gamma_{ \pm}}\right|_{L^{\infty}}$. For simplicity, we denote $|f|_{L^{\infty}(\gamma)}=|f|_{L^{\infty}\left(\gamma_{+}\right)}+|f|_{L^{\infty}\left(\gamma_{-}\right)}$.

\section{Preliminaries}

2.1. Basic properties of $L$. First of all, associated with the global Maxwellian $\mu=\mu(v)$ in (1.6), we introduce the linearized collision operator $L$ around $\mu$ and the nonlinear collision operator $\Gamma(\cdot, \cdot)$ respectively as

$$
L f=-\frac{1}{\sqrt{\mu}}\{Q(\mu, \sqrt{\mu} f)+Q(\sqrt{\mu} f, \mu)\}
$$

and

$$
\Gamma(f, f)=\frac{1}{\sqrt{\mu}} Q^{+}(\sqrt{\mu} f, \sqrt{\mu} f)-\frac{1}{\sqrt{\mu}} Q^{-}(\sqrt{\mu} f, \sqrt{\mu} f):=\Gamma^{+}(f, f)-\Gamma^{-}(f, f),
$$

where $Q^{+}$and $Q^{-}$correspond to the gain part and loss part in $Q$ in (1.2) respectively. As in [25, under the Grad's angular cutoff assumption (1.3) and (1.4), $L$ can be decomposed as $L=\nu-K$, where $\nu=\nu(v)$ is the velocity multiplication operator given by

$$
\nu(v)=\int_{\mathbb{R}^{3}} \int_{\mathbb{S}^{2}} B(|v-u|, \omega) \mu(u) \mathrm{d} \omega \mathrm{d} u \sim(1+|v|)^{\kappa},
$$


and $K=K_{1}-K_{2}$ is the integral operator in velocity given by

$$
\begin{aligned}
& \left(K_{1} f\right)(v)=\int_{\mathbb{R}^{3}} \int_{\mathbb{S}^{2}} B(|v-u|, \omega) \mu^{\frac{1}{2}}(v) \mu^{\frac{1}{2}}(u) f(u) \mathrm{d} \omega \mathrm{d} u, \\
& \left(K_{2} f\right)(v)=\int_{\mathbb{R}^{3}} \int_{\mathbb{S}^{2}} B(|v-u|, \omega) \mu^{\frac{1}{2}}(u)\left[\mu^{\frac{1}{2}}\left(u^{\prime}\right) f\left(v^{\prime}\right)+\mu^{\frac{1}{2}}\left(v^{\prime}\right) f\left(u^{\prime}\right)\right] \mathrm{d} \omega \mathrm{d} u .
\end{aligned}
$$

It is well-known that $L$ is a self-adjoint nonnegative-definite operator in $L_{v}^{2}$ space with the kernel

$$
\text { Ker } L=\operatorname{span}\left\{\phi_{0}, \cdots, \phi_{4}\right\},
$$

where $\phi_{i}=\phi_{i}(v)(i=0,1, \cdots, 4)$ are the normal orthogonal basis of the null space Ker $L$ given by

$$
\begin{aligned}
\phi_{0} & =(2 \pi)^{-\frac{1}{4}} \mu^{\frac{1}{2}}(v), \\
\phi_{i} & =(2 \pi)^{-\frac{1}{4}} v_{i} \mu^{\frac{1}{2}}(v), \quad i=1,2,3, \\
\phi_{4} & =\frac{(2 \pi)^{-\frac{1}{4}}}{\sqrt{6}}\left(|v|^{2}-3\right) \mu^{\frac{1}{2}}(v) .
\end{aligned}
$$

For each $f=f(v) \in L_{v}^{2}$, we denote the macroscopic part $P f$ as the projection of $f$ onto $\operatorname{Ker} L$, i.e.,

$$
P f=\sum_{i=0}^{4}\left\langle f, \phi_{i}\right\rangle \phi_{i},
$$

and further denote $(I-P) f=f-P f$ to be the microscopic part of $f$. It is well-known (see [28] for instance) that there is a constant $c_{0}>0$ such that

$$
\langle L f, f\rangle \geq c_{0} \int_{\mathbb{R}^{3}} \nu(v)|(I-P) f|^{2} \mathrm{~d} v .
$$

Note that $L$ has no spectral gap in case of soft potentials with $-3<\kappa<0$, particularly, the collision frequency $\nu(v)$ tends to zero as $|v| \rightarrow \infty$ due to (2.3).

2.2. Estimates on collision operators. Recall $K=K_{1}-K_{2}$ with $K_{1}$ and $K_{2}$ given in terms of (2.4) and (2.5). As in 28, it holds that

$$
K f(v)=\int_{\mathbb{R}^{3}} k(v, u) f(u) \mathrm{d} u,
$$

where the integral kernel $k(v, u)$ is real and symmetric.

Lemma 2.1 ([18]). The following estimate holds true:

$$
|k(v, u)| \leq C|v-u|^{\kappa} e^{-\frac{|v|^{2}}{4}} e^{-\frac{|u|^{2}}{4}}+\frac{C_{\kappa}}{|v-u|^{\frac{3-\kappa}{2}}} e^{-\frac{|v-u|^{2}}{8}} e^{-\frac{|v|^{2}-\left.|u|^{2}\right|^{2}}{8|v-u|^{2}}} .
$$

Moreover, it holds that

$$
\int_{\mathbb{R}^{3}}|k(v, u)|(1+|u|)^{-\beta} \mathrm{d} u \leq C_{\kappa}(1+|v|)^{-1-\beta}
$$

for any $\beta \geq 0$.

In order to deal with difficulties in the case of the soft potentials, as in [43] we introduce a smooth cutoff function $0 \leq \chi_{m}(s) \leq 1$ for $s \geq 0$ with $0<m \leq 1$ such that

$$
\chi_{m}(s)=1 \text { for } 0 \leq s \leq m ; \quad \chi_{m}(s)=0 \text { for } s \geq 2 m \text {. }
$$


Then we define

$$
\begin{aligned}
\left(K^{m} f\right)(v)= & \int_{\mathbb{R}^{3}} \int_{\mathbb{S}^{2}} B(|v-u|, \omega) \chi_{m}(|v-u|) \mu^{\frac{1}{2}}(u)\left[\mu^{\frac{1}{2}}\left(u^{\prime}\right) f\left(v^{\prime}\right)+\mu^{\frac{1}{2}}\left(v^{\prime}\right) f\left(u^{\prime}\right)\right] \mathrm{d} \omega \mathrm{d} u \\
& -\int_{\mathbb{R}^{3}} \int_{\mathbb{S}^{2}} B(|v-u|, \omega) \chi_{m}(|v-u|) \mu^{\frac{1}{2}}(v) \mu^{\frac{1}{2}}(u) f(u) \mathrm{d} \omega \mathrm{d} u \\
:= & \left(K_{2}^{m} f\right)(v)-\left(K_{1}^{m} f\right)(v),
\end{aligned}
$$

and

$$
K^{c}=K-K^{m}
$$

Similar to (2.8), we denote

$$
\left(K^{m} f\right)(v)=\int_{\mathbb{R}^{3}} k^{m}(v, u) f(u) \mathrm{d} u, \quad\left(K^{c} f\right)(v)=\int_{\mathbb{R}^{3}} k^{c}(v, u) f(u) \mathrm{d} u .
$$

In the following lemma we recall some basic estimates on $K^{m}$ and $K^{c}$, whose proof can be found in [43] and further refined in a recent work [18].

Lemma 2.2. Let $-3<\kappa<0$. Then, for any $0<m \leq 1$, it holds that

$$
\left|\left(K^{m} f\right)(v)\right| \leq C m^{3+\kappa} e^{-\frac{|v|^{2}}{6}}\|f\|_{L_{v}^{\infty}},
$$

where $C>0$ is independent of $m$. The kernels $k^{m}(v, u)$ and $k^{c}(v, u)$ in (2.10) satisfy

$$
\left|k^{m}(v, u)\right| \leq C_{\kappa}\left\{|v-u|^{\kappa}+|v-u|^{-\frac{3-\kappa}{2}}\right\} e^{-\frac{|v|^{2}+|u|^{2}}{16}},
$$

and

$$
\begin{aligned}
\left|k^{c}(v, u)\right| \leq & \frac{C_{\kappa} m^{a(\kappa-1)}}{|v-u|^{1+\frac{(1-a)}{2}(1-\kappa)}} \frac{1}{(1+|v|+|u|)^{a(1-\kappa)}} e^{-\frac{|v-u|^{2}}{10}} e^{-\frac{|v|^{2}-\left.|u|^{2}\right|^{2}}{16|v-u|^{2}}} \\
& +C|v-u|^{\kappa}\left[1-\chi_{m}(|v-u|)\right] e^{-\frac{|v|^{2}}{4}} e^{-\frac{|u|^{2}}{4}}
\end{aligned}
$$

where $0 \leq a \leq 1$ is an arbitrary constant, and $C_{\kappa}$ is a constant depending only on $\kappa$. It is worth to point out that $C_{\kappa}$ is independent of a and $m$. Moreover, by denoting

it holds that

$$
k_{w}^{c}(v, u)=k^{c}(v, u) \frac{w(v)}{w(u)},
$$

$$
\int_{\mathbb{R}^{3}}\left|k_{w}^{c}(v, u)\right| e^{\frac{|v-u|^{2}}{32}} \mathrm{~d} u \leq C m^{\kappa-1}(1+|v|)^{\kappa-2},
$$

and

$$
\int_{\mathbb{R}^{3}}\left|k_{w}^{c}(v, u)\right| e^{\frac{|v-u|^{2}}{32}} \mathrm{~d} u \leq C(1+|v|)^{-1},
$$

where $C>0$ is independent of $m$.

Furthermore, we need the following two lemmas for the later use.

Lemma 2.3 (43]). Assume (1.10), then for any $\eta>0$, it holds that

$$
\left|\left\langle e^{\frac{w}{2}|\cdot|^{\zeta}} K f, f\right\rangle\right| \leq\left\|e^{\frac{w}{4}|\cdot|^{\zeta}} f\right\|_{\nu}\left(\eta\left\|e^{\frac{w}{4}|\cdot|^{\zeta}} f\right\|_{\nu}+C_{\eta}\left\|\mathbf{1}_{\left\{|\cdot| \leq C_{\eta}\right\}} f\right\|_{L^{2}}\right) .
$$

Lemma 2.4 ([36]). It holds that

$$
\left\|\nu^{-1} w \Gamma\left(f_{1}, f_{2}\right)\right\|_{L_{v}^{\infty}} \leq C\left\|w f_{1}\right\|_{L_{v}^{\infty}} \cdot\left\|w f_{2}\right\|_{L_{v}^{\infty}} .
$$

In the end we conclude this subsection with the following $L^{p}(p>1)$ estimate on the nonlinear collision term, which will be used in Section 5. 
Lemma 2.5. Let $1<p<\infty$ and $w_{\beta}:=\left(1+|v|^{2}\right)^{\frac{\beta}{2}}$ with $\beta p>3$. Then it holds that

$$
\left\|\nu^{-1 / p^{\prime}} \Gamma(f, g)\right\|_{L_{v}^{p}} \leq C \min \left\{\left\|w_{\beta} f\right\|_{L_{v}^{\infty}} \cdot\left\|\nu^{1 / p} g\right\|_{L_{v}^{p}},\left\|w_{\beta} g\right\|_{L_{v}^{\infty}} \cdot\left\|\nu^{1 / p} f\right\|_{L_{v}^{p}}\right\},
$$

where $p^{\prime}$ is the conjugate of $p$ satisfying $1 / p+1 / p^{\prime}=1$.

Proof. We first consider the loss part. By Hölder inequality, we have

$$
\begin{aligned}
\left\|\nu^{-1 / p^{\prime}} \Gamma^{-}(f, g)\right\|_{L_{v}^{p}}^{p} & \leq \int_{\mathbb{R}^{3}} \nu(v)^{-(p-1)}|f(v)|^{p} \mathrm{~d} v\left(\int_{\mathbb{R}^{3}} \int_{\mathbb{S}^{2}} B(v-u, \omega) \sqrt{\mu(u)}|g(u)|^{p} \mathrm{~d} \omega \mathrm{d} u\right) \\
& \times\left(\int_{\mathbb{R}^{3}} \int_{\mathbb{S}^{2}} B(v-u, \omega) \sqrt{\mu(u)} \mathrm{d} \omega \mathrm{d} u\right)^{p-1} \\
\leq & C \int_{\mathbb{R}^{3}} \int_{\mathbb{R}^{3}} \int_{\mathbb{S}^{2}} B(v-u, \omega) \sqrt{\mu(u)}|g(u)|^{p}|f(v)|^{p} \mathrm{~d} \omega \mathrm{d} u \mathrm{~d} v .
\end{aligned}
$$

Without loss of generality, we assume that

$$
\left\|w_{\beta} f\right\|_{L_{v}^{\infty}} \cdot\left\|\nu^{1 / p} g\right\|_{L_{v}^{p}} \geq\left\|w_{\beta} g\right\|_{L_{v}^{\infty}} \cdot\left\|\nu^{1 / p} f\right\|_{L_{v}^{p}} .
$$

Then from (2.18), it holds that

$$
\begin{aligned}
\left\|\nu^{-1 / p^{\prime}} \Gamma^{-}(f, g)\right\|_{L_{v}^{p}}^{p} & \leq C\left\|w_{\beta} g\right\|_{L_{v}^{\infty}}^{p} \cdot \int_{\mathbb{R}^{3}}|f(v)|^{p} \mathrm{~d} v \int_{\mathbb{R}^{3}} \int_{\mathbb{S}^{2}} B(v-u, \omega)\left(1+|u|^{2}\right)^{-\frac{\beta p}{2}} \mathrm{~d} \omega \mathrm{d} u \\
& \leq C\left\|w_{\beta} g\right\|_{L_{v}^{\infty}}^{p} \cdot\left\|\nu^{1 / p} f\right\|_{L_{v}^{p}}^{p} \\
& \leq C \min \left\{\left\|w_{\beta} f\right\|_{L_{v}^{\infty}} \cdot\left\|\nu^{1 / p} g\right\|_{L_{v}^{p}},\left\|w_{\beta} g\right\|_{L_{v}^{\infty}} \cdot\left\|\nu^{1 / p} f\right\|_{L_{v}^{p}}\right\}^{p} .
\end{aligned}
$$

Similarly, for the gain term $\Gamma^{+}(f, g)$, by Hölder inequality, we have

$$
\begin{aligned}
\left\|\nu^{-1 / p^{\prime}} \Gamma^{+}(f, g)\right\|_{L_{v}^{p}}^{p} & \leq \int_{\mathbb{R}^{3}} \nu(v)^{-(p-1)} \mathrm{d} v\left(\int_{\mathbb{R}^{3}} \int_{\mathbb{S}^{2}} B(v-u, \omega) \sqrt{\mu(u)}\left|f\left(v^{\prime}\right)\right|^{p}\left|g\left(u^{\prime}\right)\right|^{p} \mathrm{~d} \omega \mathrm{d} u\right) \\
& \times\left(\int_{\mathbb{R}^{3}} \int_{\mathbb{S}^{2}} B(v-u, \omega) \sqrt{\mu(u)} \mathrm{d} \omega \mathrm{d} u\right)^{p-1} \\
\leq & C \int_{\mathbb{R}^{3}} \int_{\mathbb{R}^{3}} \int_{\mathbb{S}^{2}} B(v-u, \omega) \sqrt{\mu(u)}\left|g\left(u^{\prime}\right)\right|^{p}\left|f\left(v^{\prime}\right)\right|^{p} \mathrm{~d} \omega \mathrm{d} u \mathrm{~d} v .
\end{aligned}
$$

Making change of variable $(v, u) \rightarrow\left(v^{\prime}, u^{\prime}\right)$, it follows that

$$
\left\|\nu^{-1 / p^{\prime}} \Gamma^{+}(f, g)\right\|_{L_{v}^{p}}^{p} \leq C \int_{\mathbb{R}^{3}} \int_{\mathbb{R}^{3}} \int_{\mathbb{S}^{2}} B(v-u, \omega)|g(u)|^{p}|f(v)|^{p} \mathrm{~d} \omega \mathrm{d} u \mathrm{~d} v .
$$

By the same argument as in (2.19), one has

$$
\left\|\nu^{-1 / p^{\prime}} \Gamma^{+}(f, g)\right\|_{L_{v}^{p}}^{p} \leq C \min \left\{\left\|w_{\beta} f\right\|_{L_{v}^{\infty}} \cdot\left\|\nu^{1 / p} g\right\|_{L_{v}^{p}},\left\|w_{\beta} g\right\|_{L_{v}^{\infty}} \cdot\left\|\nu^{1 / p} f\right\|_{L_{v}^{p}}\right\}^{p}
$$

From this and (2.19), we prove (2.17). Therefore, the proof of Lemma 2.5 is complete.

2.3. Pointwise weighted estimates on nonlinear term $\Gamma(f, f)$. Recall (1.9), (1.10), and (2.2). We shall give the estimates on the upper bound of $\left|w(v) \Gamma^{ \pm}(f, f)(v)\right|$ for pointwise $v$ in terms of the product of the weighted $L^{\infty}$ norm and the $L^{p}$ norm for a suitable $p>1$ which is finite. These estimates will play an essential role in the proof of Theorem 1.3 treating the initial data of large oscillations but with small $L^{p}$ perturbations. 
Lemma 2.6. Let $-3<\kappa<0, \max \left\{\frac{3}{2}, \frac{3}{3+\kappa}\right\}<p<\infty,(\varpi, \zeta)$ belong (1.10), and $\beta>4$. Then, for each $v \in \mathbb{R}^{3}$, it holds that:

$$
\begin{aligned}
& \left|w \Gamma^{-}(f, f)(v)\right| \leq C \nu(v)\|w f\|_{L_{v}^{\infty}} \cdot\left(\int_{\mathbb{R}^{3}}|f(u)|^{p} \mathrm{~d} u\right)^{\frac{1}{p}}, \\
& \left|w \Gamma^{+}(f, f)(v)\right| \leq C \nu(v)\|w f\|_{L_{v}^{\infty}} \cdot\left(\int_{\mathbb{R}^{3}}|w f(u)|^{p}(1+|u|)^{-p(\beta-4)-4} \mathrm{~d} u\right)^{\frac{1}{p}},
\end{aligned}
$$

where the generic constant $C>0$ is independent of $v$.

Proof. First, we consider (2.20) regarding the estimate on the loss term. Indeed, it follows from Hölder inequality that

$$
\begin{aligned}
\left|w \Gamma^{-}(f, f)(v)\right| & \leq|w f(v)| \int_{\mathbb{R}^{3}} \int_{\mathbb{S}^{2}} B(|v-u|, \omega) \mu^{\frac{1}{2}}(u)|f(u)| \mathrm{d} u \mathrm{~d} \omega \\
& \leq C\|w f\|_{L_{v}^{\infty}} \cdot\left(\int_{\mathbb{R}^{3}}|f(u)|^{p} \mathrm{~d} u\right)^{\frac{1}{p}} \cdot\left(\int_{\mathbb{R}^{3}}|v-u|^{\frac{p \kappa}{p-1}} \mu^{\frac{p}{2(p-1)}}(u) \mathrm{d} u\right)^{\frac{p}{p-1}} \\
& \leq C \nu(v)\|w f\|_{L_{v}^{\infty}} \cdot\left(\int_{\mathbb{R}^{3}}|f(u)|^{p} \mathrm{~d} u\right)^{\frac{1}{p}}
\end{aligned}
$$

where we have used the fact that $\frac{p \kappa}{p-1}>-3$ due to $p>\frac{3}{3+\kappa}$. Then, (2.201) is proved.

To prove (2.21) for the gain term, we denote

$$
\hat{w}(v):=e^{\varpi|v|^{\zeta}},
$$

which is the pure exponential factor of $w(v)$, and also set $g(v)=\hat{w}(v)|f(v)|$. Since $|u|^{2}+|v|^{2}=$ $\left|u^{\prime}\right|^{2}+\left|v^{\prime}\right|^{2}$, we have either $\left|u^{\prime}\right|^{2} \geq \frac{1}{2}\left(|u|^{2}+|v|^{2}\right)$ or $\left|v^{\prime}\right|^{2} \geq \frac{1}{2}\left(|u|^{2}+|v|^{2}\right)$. Without loss of generality, we may assume that $\left|v^{\prime}\right|^{2} \geq \frac{1}{2}\left(|u|^{2}+|v|^{2}\right)$, and then we have

$$
\left|w \Gamma^{+}(f, f)(v)\right| \leq C \hat{w}(v)\|w f\|_{L_{v}^{\infty}} \cdot \int_{\mathbb{R}^{3}} \int_{\mathbb{S}^{2}} B(|v-u|, \omega) \mu^{\frac{1}{2}}(u)\left|g\left(v^{\prime}\right)\right| \hat{w}\left(v^{\prime}\right)^{-1} \hat{w}\left(u^{\prime}\right)^{-1} \mathrm{~d} u \mathrm{~d} \omega .
$$

Note that in virtue of $0<\zeta \leq 2$, it holds that

$$
\hat{w}(v)=e^{\varpi|v|^{\zeta}} \leq e^{\varpi\left(|u|^{2}+|v|^{2}\right)^{\frac{\zeta}{2}}}=e^{\varpi\left(\left|u^{\prime}\right|^{2}+\left|v^{\prime}\right|^{2}\right)^{\frac{\zeta}{2}}} \leq e^{\varpi\left(\left|u^{\prime}\right|^{\zeta}+\left|v^{\prime}\right|^{\zeta}\right)}=\hat{w}\left(v^{\prime}\right) \hat{w}\left(u^{\prime}\right) .
$$

Using this, 2.22) gives that

$$
\left|w \Gamma^{+}(f, f)(v)\right| \leq C\|w f\|_{L_{v}^{\infty}} \cdot \int_{\mathbb{R}^{3}} \int_{\mathbb{S}^{2}} B(|v-u|, \omega) \mu^{\frac{1}{2}}(u)\left|g\left(v^{\prime}\right)\right| \mathrm{d} u \mathrm{~d} \omega .
$$

To further estimate the integral term on the right-hand side of (2.23), we denote $z=u-v$, $z_{\|}=\{(u-v) \cdot \omega\} \omega$, and $z_{\perp}=z-z_{\|}$, then the collision kernel can be estimated as

$$
B(|v-u|, \omega) \leq C\left|z_{\|}\right|\left(\left|z_{\|}\right|^{2}+\left|z_{\perp}\right|^{2}\right)^{\frac{\kappa-1}{2}} .
$$

Plugging (2.24) back to (2.23) and making change of variable $u \rightarrow z,\left|w \Gamma^{+}(f, f)(v)\right|$ can be further bounded by

$$
C\|w f\|_{L_{v}^{\infty}} \cdot \int_{\mathbb{R}^{3}} \int_{\mathbb{S}^{2}}\left|z_{\|}\right| \cdot|z|^{\kappa-1} g\left(v+z_{\|}\right) \mu^{\frac{1}{2}}(v+z) \mathrm{d} z \mathrm{~d} \omega .
$$

By writing $\mathrm{d} z \mathrm{~d} \omega=\frac{2}{\left|z_{\|}\right|^{2}} \mathrm{~d} z_{\|} \mathrm{d} z_{\perp}$, we derive that the above term is bounded by

$$
C\|w f\|_{L_{v}^{\infty}} \cdot \int_{\mathbb{R}^{3}}\left|z_{\|}\right|^{-1} g\left(v+z_{\|}\right) \mathrm{d} z_{\|} \int_{\Pi_{\perp}}\left(\left|z_{\|}\right|^{2}+\left|z_{\perp}\right|^{2}\right)^{\frac{\kappa-1}{2}} \mu^{\frac{1}{2}}\left(v_{\perp}+z_{\perp}\right) \mathrm{d} z_{\perp},
$$

where $\Pi_{\perp}=\left\{z_{\perp} \in \mathbb{R}^{3}: z_{\|} \cdot z_{\perp}=0\right\}$ and $v_{\perp}=\frac{v \cdot z_{\perp}}{|v| \cdot\left|z_{\perp}\right|} z_{\perp}$ is the projection of $v$ to $\Pi_{\perp}$. To estimate (2.25), we divide it by two cases. 
Case 1: $-1<\kappa<0$. In this case, thanks to $-2<\kappa-1<-1$, we have

$$
\int_{\Pi_{\perp}}\left(\left|z_{\|}\right|^{2}+\left|z_{\perp}\right|^{2}\right)^{\frac{\kappa-1}{2}} \mu^{\frac{1}{2}}\left(v_{\perp}+z_{\perp}\right) \mathrm{d} z_{\perp} \leq \int_{\Pi_{\perp}}\left|z_{\perp}\right|^{\kappa-1} \mu^{\frac{1}{2}}\left(v_{\perp}+z_{\perp}\right) \mathrm{d} z_{\perp} \leq C,
$$

for a finite constant $C>0$. Then it holds that for $p>\frac{3}{2}$,

$$
\begin{aligned}
\left|w \Gamma^{+}(f, f)(v)\right| & \leq C \int_{\mathbb{R}^{3}}\left|z_{\|}\right|^{-1} g\left(v+z_{\|}\right) \mathrm{d} z_{\|}=C \int_{\mathbb{R}^{3}}|u-v|^{-1} g(u) \mathrm{d} u \\
& \leq C\left(\int_{\mathbb{R}^{3}} \frac{(1+|u|)^{-4}}{|u-v|^{\frac{p}{p-1}}}\right)^{\frac{p-1}{p}}\left(\int_{\mathbb{R}^{3}}|g(u)|^{p}(1+|u|)^{4 p-4} \mathrm{~d} u\right)^{\frac{1}{p}} \\
& \leq C(1+|v|)^{-1}\left(\int_{\mathbb{R}^{3}}|g(u)|^{p}(1+|u|)^{4 p-4} \mathrm{~d} u\right)^{\frac{1}{p}} .
\end{aligned}
$$

Case 2 : $-3<\kappa \leq-1$. To avoid the higher singularity on the right hand side of (2.25), we choose $0<\epsilon=\epsilon(p, \kappa)<\min \left\{3+\frac{p \kappa}{p-1}, \frac{2 p}{p-1}\right\}$ and bound (2.25) in terms of

$$
\begin{aligned}
\left|w \Gamma^{+}(f, f)\right| & \leq C\|w f\|_{L_{v}^{\infty}} \cdot \int_{\mathbb{R}^{3}}\left|z_{\|}\right|^{\kappa-\frac{(p-1) \epsilon}{p}} g\left(v+z_{\|}\right) \mathrm{d} z_{\|} \int_{\Pi_{\perp}}\left|z_{\perp}\right|^{-2+\frac{(p-1) \epsilon}{p}} \mu^{\frac{1}{2}}\left(v_{\perp}+z_{\perp}\right) \mathrm{d} z_{\perp} \\
& \leq C\|w f\|_{L_{v}^{\infty}} \cdot \int_{\mathbb{R}^{3}}\left|z_{\|}\right|^{\kappa-\frac{(p-1) \epsilon}{p}} g\left(v+z_{\|}\right) \mathrm{d} z_{\|}
\end{aligned}
$$

where in the second line we have used the fact that

$$
\int_{\Pi_{\perp}}\left|z_{\perp}\right|^{-2+\frac{(p-1) \epsilon}{p}} \mu^{\frac{1}{2}}\left(v_{\perp}+z_{\perp}\right) \mathrm{d} z_{\perp}<\infty .
$$

Making change of variable $z_{\|}+v \rightarrow u$ and using Hölder's inequality, it holds that

$$
\begin{aligned}
\int_{\mathbb{R}^{3}}\left|z_{\|}\right|^{\kappa-\frac{(p-1) \epsilon}{p}} g\left(v+z_{\|}\right) d z_{\|} & =\int_{\mathbb{R}^{3}}|u-v|^{\kappa-\frac{(p-1) \epsilon}{p}} g(u) \mathrm{d} u \\
\leq & {\left[\int_{\mathbb{R}^{3}} \frac{(1+|u|)^{-4}}{|u-v|^{\epsilon+\frac{p|\kappa|}{p-1}}} \mathrm{~d} u\right]^{\frac{p-1}{p}} \times\left[\int_{\mathbb{R}^{3}}|g(u)|^{p}(1+|u|)^{4 p-4} \mathrm{~d} u\right]^{\frac{1}{p}} . }
\end{aligned}
$$

Since $0<\epsilon+\frac{p|\kappa|}{p-1}<3$, the right hand side of (2.28) can be further bounded by

$$
C(1+|v|)^{\kappa-\frac{p-1}{p} \epsilon}\left[\int_{\mathbb{R}^{3}}|g(u)|^{p}(1+|u|)^{4 p-4} \mathrm{~d} u\right]^{\frac{1}{p}}
$$

which combining with (2.27) and (2.26), immediately yields (2.21). Hence the proof of Lemma 2.6 is complete.

\section{Steady PRoblem}

To construct the solution to the steady Boltzmann equation (1.8) and (1.5), we first consider the approximate linearized steady problem

$$
\left\{\begin{array}{l}
\varepsilon f+v \cdot \nabla_{x} f+L f=g \\
\left.f(x, v)\right|_{\gamma_{-}}=P_{\gamma} f+r
\end{array}\right.
$$

where $P_{\gamma} f$ is defined as

$$
P_{\gamma} f(x, v)=\mu^{\frac{1}{2}}(v) \int_{v^{\prime} \cdot n(x)>0} f\left(x, v^{\prime}\right) \mu^{\frac{1}{2}}\left(v^{\prime}\right)\left\{v^{\prime} \cdot n(x)\right\} \mathrm{d} v^{\prime} .
$$


As in [21, the penalization term $\varepsilon f$ is used to guarantee the conservation of mass. Recall the weight function $w(v)$ defined by (1.9) with (1.10). We also define

$$
h(x, v):=w(v) f(x, v) \text {, }
$$

then (3.1) can be rewritten as

$$
\left\{\begin{array}{l}
\varepsilon h+v \cdot \nabla_{x} h+\nu(v) h=K_{w} h+w g, \\
\left.h(x, v)\right|_{\gamma_{-}}=\frac{1}{\tilde{w}(v)} \int_{v^{\prime} \cdot n(x)>0} h\left(x, v^{\prime}\right) \tilde{w}\left(v^{\prime}\right) \mathrm{d} \sigma^{\prime}+w r(x, v),
\end{array}\right.
$$

where

$$
\tilde{w}(v) \equiv \frac{1}{w(v) \mu^{\frac{1}{2}}(v)}, \quad K_{w} h=w K\left(\frac{h}{w}\right) .
$$

3.1. A priori $L^{\infty}$ estimate. For the approximate steady Boltzmann equation (3.3), the most difficult part is to obtain the $L^{\infty}$-bound due to the degeneration of frequency $\nu(v)$ as $|v| \rightarrow \infty$. To overcome this difficulty, the main idea is to introduce a new characteristic line.

Definition 3.1. Given $(t, x, v)$, let $[\hat{X}(s), V(s)]$ be the backward bi-characteristics for the steady Boltzmann equation (1.8), which is determined by

$$
\left\{\begin{array}{l}
\frac{d \hat{X}(s)}{d s}=\left(1+|V(s)|^{2}\right)^{\frac{|\kappa|}{2}} V(s):=\hat{V}(s), \\
\frac{d V(s)}{d s}=0 \\
{[\hat{X}(t), V(t)]=[x, v] .}
\end{array}\right.
$$

The solution is then given by

$$
[\hat{X}(s ; t, x, v), V(s ; t, x, v)]=[x-\hat{v}(t-s), v], \quad \hat{v}:=\left(1+|v|^{2}\right)^{\frac{|\kappa|}{2}} v,
$$

which is called the speeded backward bi-characteristic for the problem (1.8).

We note that compared to the usual characteristic line as used in the time-evolutionary case, the particle along (3.4) or (3.5) with given $(x, v)$ travels with the velocity $\hat{v}$ which has the much faster speed than $|v|$ itself for $|v|$ for large velocity. This is the key idea to overcome the difficulty of soft potentials in treating the steady problem on the Boltzmann equation.

In terms of the speeded backward bi-characteristic, we need to redefine the corresponding backward exit time et al. Indeed, for each $(x, v)$ with $x \in \bar{\Omega}$ and $v \neq 0$, we define the backward exit time $\hat{t}_{\mathbf{b}}(x, v) \geq 0$ to be the last moment at which the back-time straight line $[\hat{X}(-\tau ; 0, x, v), V(-\tau ; 0, x, v)]$ remains in $\bar{\Omega}$ :

$$
\hat{t}_{\mathbf{b}}(x, v)=\sup \{s \geq 0: x-\hat{v} \tau \in \bar{\Omega} \text { for } 0 \leq \tau \leq s\} .
$$

We therefore have $x-\hat{t}_{\mathbf{b}} \hat{v} \in \partial \Omega$ and $\xi\left(x-\hat{t}_{\mathbf{b}} \hat{v}\right)=0$. We also define

$$
\hat{x}_{\mathbf{b}}(x, v)=x-\hat{t}_{\mathbf{b}} \hat{v} \in \partial \Omega \text {. }
$$

Note that the fact that $v \cdot n\left(\hat{x}_{\mathbf{b}}\right)=v \cdot n\left(\hat{x}_{\mathbf{b}}(x, v)\right) \leq 0$ always holds true.

Let $x \in \bar{\Omega},(x, v) \notin \gamma_{0} \cup \gamma_{-}$and $\left(t_{0}, x_{0}, v_{0}\right)=(t, x, v)$. For $v_{k+1} \in \hat{\mathcal{V}}_{k+1}:=\left\{v_{k+1} \cdot n\left(\hat{x}_{k+1}\right)>0\right\}$, the back-time cycle is defined as

$$
\left\{\begin{array}{l}
\hat{X}_{c l}(s ; t, x, v)=\sum_{k} \mathbf{1}_{\left[\hat{t}_{k+1}, \hat{t}_{k}\right)}(s)\left\{\hat{x}_{k}-\hat{v}_{k}\left(\hat{t}_{k}-s\right)\right\}, \\
V_{c l}(s ; t, x, v)=\sum_{k} \mathbf{1}_{\left[\hat{t}_{k+1}, \hat{t}_{k}\right)}(s) v_{k},
\end{array}\right.
$$

with

$$
\left(\hat{t}_{k+1}, \hat{x}_{k+1}, v_{k+1}\right)=\left(\hat{t}_{k}-\hat{t}_{\mathbf{b}}\left(\hat{x}_{k}, v_{k}\right), \hat{x}_{\mathbf{b}}\left(\hat{x}_{k}, v_{k}\right), v_{k+1}\right) .
$$


We also define the iterated integral

$$
\int_{\Pi_{j=1}^{k-1} \hat{\mathcal{V}}_{j}} \Pi_{j=1}^{k-1} \mathrm{~d} \hat{\sigma}_{j}:=\int_{\hat{\mathcal{V}}_{1}} \cdots\left\{\int_{\hat{\mathcal{V}}_{k-1}} \mathrm{~d} \hat{\sigma}_{k-1}\right\} \cdots \mathrm{d} \hat{\sigma}_{1},
$$

where

$$
\mathrm{d} \hat{\sigma}_{j}:=\mu\left(v_{j}\right)\left\{n\left(\hat{x}_{j}\right) \cdot v_{j}\right\}, \quad j=1, \cdots, k-1
$$

are probability measures.

Lemma 3.2. Let $(\eta, \zeta)$ belong to

$$
\{\zeta=2,0 \leq \eta<1 / 2\} \cup\{0 \leq \zeta<2, \eta \geq 0\} .
$$

For $T_{0}>0$ sufficiently large, there exist constants $\hat{C}_{1}$ and $\hat{C}_{2}$ independent of $T_{0}$ such that for $k=\hat{C}_{1} T_{0}^{\frac{5}{4}}$ and $(t, x, v) \in\left[0, T_{0}\right] \times \bar{\Omega} \times \mathbb{R}^{3}$, it holds that

$$
\int_{\Pi_{j=1}^{k-1} \hat{\mathcal{V}}_{j}} \mathbf{1}_{\left\{\hat{t}_{k}>0\right\}} \Pi_{j=1}^{k-1} e^{\eta\left|v_{j}\right|^{\zeta}} \mathrm{d} \hat{\sigma}_{j} \leq\left(\frac{1}{2}\right)^{\hat{C}_{2} T_{0}^{\frac{5}{4}}} .
$$

Proof. We take $\epsilon>0$ small enough, and define the non-grazing sets

$$
\hat{\mathcal{V}}_{j}^{\epsilon}=\left\{v_{j} \in \hat{\mathcal{V}}_{j}: v_{j} \cdot n\left(\hat{x}_{j}\right) \geq \epsilon \text { and }\left|v_{j}\right| \leq \frac{1}{\epsilon}\right\}, \quad j \geq 1 .
$$

Then a direct calculation shows that

$$
\int_{\hat{\mathcal{V}}_{j} \backslash \hat{\mathcal{V}}_{j}^{\epsilon}} e^{\eta\left|v_{j}\right|^{\zeta} \mathrm{d} \hat{\sigma}_{j} \leq C \epsilon}
$$

where the constant $C>0$ is independent of $j$. By similar arguments as in [29, Lemma 2], one can prove

$$
\hat{t}_{j}-\hat{t}_{j+1} \geq \frac{\left|v_{j} \cdot n\left(\hat{x}_{j}\right)\right|}{C_{\Omega}\left|v_{j}\right|^{2}\left(1+\left|v_{j}\right|^{2}\right)^{\frac{|\kappa|}{2}}}
$$

with a positive constant $C_{\Omega}$ depending only on the domain. If $v_{j} \in \hat{\mathcal{V}}_{j}$, then we have $\hat{t}_{j}-\hat{t}_{j+1} \geq$ $\frac{\epsilon^{3+|\kappa|}}{C_{\Omega}}$. Therefore, if $\hat{t}_{k}=\hat{t}_{k}\left(t, x, v, v_{1}, \cdots, v_{k-1}\right)>0$, there can be at most $\left[\frac{C_{\Omega} T_{0}}{\epsilon^{3+|\kappa|}}\right]+1$ number of $v_{j} \in \hat{\mathcal{V}}_{j}^{\epsilon}$ for $1 \leq j \leq k-1$. Hence we have

$$
\begin{aligned}
& \int_{\Pi_{j=1}^{k-1} \hat{\mathcal{V}}_{j}} \mathbf{1}_{\left\{\hat{t}_{k}>0\right\}} \Pi_{j=1}^{k-1} e^{\eta\left|v_{j}\right|^{\zeta} \mathrm{d} \hat{\sigma}_{j}} \\
& \leq \sum_{n=1}^{\left[\frac{C_{\Omega} T_{0}}{\epsilon^{+}+\mid \kappa \kappa}\right]+1} \int_{\left\{\text {There are n number } v_{j} \in \hat{\mathcal{V}}_{j}^{\epsilon} \text { for some } 1 \leq j \leq k-1\right\}} \prod_{j=1}^{k-1} e^{\eta\left|v_{j}\right|^{\zeta}} \mathrm{d} \hat{\sigma}_{j} \\
& \leq \sum_{n=1}^{\left[\frac{C_{\Omega} T_{0}}{\left.\epsilon^{3+|\kappa| \kappa \mid}\right]+1}\right.}\left(\begin{array}{c}
k-1 \\
n
\end{array}\right)\left|\sup _{j} \int_{\hat{\mathcal{V}}_{j}^{\epsilon}} e^{\eta\left|v_{j}\right|^{\zeta}} \mathrm{d} \hat{\sigma}_{j}\right|^{n} \cdot \mid \sup _{j} \int_{\hat{\mathcal{V}}_{j} \backslash \hat{\mathcal{V}}_{j}^{\epsilon}} e^{\left.\eta\left|v_{j}\right|^{\zeta} \mathrm{d} \hat{\sigma}_{j}\right|^{k-1-n}} \\
& \leq\left(\left[\frac{C_{\Omega} T_{0}}{\epsilon^{3+|\kappa|}}\right]+1\right) \cdot(k-1)^{\left[\frac{C_{\Omega} T_{0}}{\epsilon^{3+|\kappa|}}\right]+1}(C \epsilon)^{k-1-2\left[\frac{C_{\Omega} T_{0}}{\epsilon^{3+|\kappa|}}+1\right]} .
\end{aligned}
$$


One can take $k-1=N\left(\left[\frac{C_{\Omega} T_{0}}{\epsilon^{3+|\kappa|}}\right]+1\right)$ with $\left[\frac{C_{\Omega} T_{0}}{\epsilon^{3+|\kappa|}}\right] \geq 1$ and $N>2(3+|\kappa|)$, so that (3.8) can be bounded as

$$
\begin{aligned}
\int_{\Pi_{j=1}^{k-1} \hat{\mathcal{V}}_{j}} \mathbf{1}_{\left\{\hat{t}_{k}>0\right\}} \Pi_{j=1}^{k-1} e^{\eta\left|v_{j}\right|^{\zeta} \mathrm{d} \hat{\sigma}_{j}} & \leq\left\{2 N\left(\left[\frac{C_{\Omega} T_{0}}{\epsilon^{3+|\kappa|}}\right]+1\right)\right\}^{\left[\frac{C_{\Omega} T_{0}}{\epsilon^{3+|\kappa|}}\right]+1}(C \epsilon)^{\frac{N}{2}\left(1+\left[\frac{C_{\Omega} T_{0}}{\epsilon^{3+\mid \kappa \kappa}}\right]\right)} \\
& \leq\left\{4 N\left[\frac{C_{\Omega} T_{0}}{\epsilon^{3+|\kappa|}}\right](C \epsilon)^{\frac{N}{2}}\right\}^{\left[\frac{C_{\Omega} T_{0}}{\epsilon^{3+|\kappa|}}\right]+1} \\
& \leq\left\{C_{\Omega, N} \cdot T_{0} \cdot \epsilon^{\frac{N}{2}-3-|\kappa|}\right\}^{\left[\frac{C_{\Omega} T_{0}}{\epsilon^{3+|\kappa|}}\right]+1} .
\end{aligned}
$$

We choose

$$
\epsilon=\left(\frac{1}{2 C_{\Omega, N} \cdot T_{0}}\right)^{\frac{1}{\frac{N}{2}-3-|\kappa|}}
$$

such that $C_{\Omega, N} \cdot T_{0} \cdot \epsilon^{\frac{N}{2}-3-|\kappa|}=1 / 2$. Note that for large $T_{0}$, it holds that $\epsilon>0$ is small, and

$$
\left[\frac{C_{\Omega} T_{0}}{\epsilon^{3+|\kappa|}}\right]+1 \cong C_{\Omega, N} T_{0}^{1+\frac{3+|\kappa|}{\frac{N}{2}-3-|\kappa|}}
$$

Finally, we take $N=6(3+|\kappa|)$, so that $\left[\frac{C_{\Omega} T_{0}}{\epsilon^{3+|\kappa|}}\right]+1=C T_{0}^{\frac{5}{4}}$ and

$$
k=6(3+|\kappa|)\left\{\left[\frac{C_{\Omega} T_{0}}{\epsilon^{3+|\kappa|}}\right]+1\right\}+1=C T_{0}^{\frac{5}{4}} .
$$

Therefore, (3.7) follows. This completes the proof of Lemma 3.2.

Along the back-time cycle (3.6), we can represent the solution of (3.3) in a mild formulation which enables us to get the $L^{\infty}$ bound of solutions in the steady case. Indeed, for later use, we consider the following iterative linear problems involving a parameter $\lambda \in[0,1]$ :

$$
\left\{\begin{array}{l}
\varepsilon h^{i+1}+v \cdot \nabla_{x} h^{i+1}+\nu(v) h^{i+1}=\lambda K_{w}^{m} h^{i}+\lambda K_{w}^{c} h^{i}+w g \\
\left.h^{i+1}(x, v)\right|_{\gamma_{-}}=\frac{1}{\tilde{w}(v)} \int_{v^{\prime} \cdot n(x)>0} h^{i}\left(x, v^{\prime}\right) \tilde{w}\left(v^{\prime}\right) \mathrm{d} \sigma^{\prime}+w(v) r(x, v),
\end{array}\right.
$$

for $i=0,1,2, \cdots$, where $h^{0}=h^{0}(x, v)$ is given. For the mild formulation of (3.9), we have the following lemma whose proof is omitted for brevity as it is similar to that in [29].

Lemma 3.3. Let $0 \leq \lambda \leq 1$. Denote $\hat{\nu}(v):=\left(1+|v|^{2}\right)^{\frac{|\kappa|}{2}}[\varepsilon+\nu(v)]$. For each $t \in\left[0, T_{0}\right]$ and for each $(x, v) \in \bar{\Omega} \times \mathbb{R}^{3} \backslash\left(\gamma_{0} \cup \gamma_{-}\right)$, we have

$$
h^{i+1}(x, v)=\sum_{n=1}^{4} J_{n}+\sum_{n=5}^{14} \mathbf{1}_{\left\{t_{1}>s\right\}} J_{n},
$$


with

$$
\begin{aligned}
& J_{1}=\mathbf{1}_{\left\{\hat{t}_{1} \leq 0\right\}} e^{-\hat{\nu}(v) t} h^{i+1}(x-\hat{v} t), \\
& J_{2}+J_{3}+J_{4}=\int_{\max \left\{\hat{t}_{1}, 0\right\}}^{t} e^{-\hat{\nu}(v)(t-s)}\left(1+|v|^{2}\right)^{\frac{|\kappa|}{2}}\left[\lambda K_{w}^{m} h^{i}+\lambda K_{w}^{c} h^{i}+w g\right](x-\hat{v}(t-s), v) \mathrm{d} s, \\
& J_{5}=e^{-\hat{\nu}(v)\left(t-\hat{t}_{1}\right)} w(v) r\left(\hat{x}_{1}, v\right), \\
& J_{6}=\frac{e^{-\hat{\nu}(v)\left(t-\hat{t}_{1}\right)}}{\tilde{w}(v)} \int_{\Pi_{j=1}^{k-1} \hat{\nu}_{j}} \sum_{l=1}^{k-2} \mathbf{1}_{\left\{\hat{t}_{l+1}>0\right\}} w\left(v_{l}\right) r\left(\hat{x}_{l+1}, v_{l}\right) \mathrm{d} \hat{\Sigma}_{l}\left(\hat{t}_{l+1}\right), \\
& J_{7}=\frac{e^{-\hat{\nu}(v)\left(t-\hat{t}_{1}\right)}}{\tilde{w}(v)} \int_{\Pi_{j=1}^{k-1} \hat{\nu}_{j}} \sum_{l=1}^{k-1} \mathbf{1}_{\left\{\hat{t}_{l+1} \leq 0<\hat{t}_{l}\right\}} h^{i+1-l}\left(\hat{x}_{l}-\hat{v}_{l} \hat{t}_{l}, v_{l}\right) \mathrm{d} \hat{\Sigma}_{l}(0), \\
& J_{8}+J_{9}+J_{10}=\frac{e^{-\hat{\nu}(v)\left(t-\hat{t}_{1}\right)}}{\tilde{w}(v)} \int_{\Pi_{j=1}^{k-1} \hat{\nu}_{j}} \sum_{l=1}^{k-1} \int_{0}^{\hat{t}_{l}} \mathbf{1}_{\left\{\hat{t}_{l+1} \leq 0<\hat{t}_{l}\right\}} \\
& \quad \times\left(1+\left|v_{l}\right|^{2}\right)^{\frac{|\kappa|}{2}}\left[\lambda K_{w}^{m} h^{i-l}+\lambda K_{w}^{c} h^{i-l}+w g\right]\left(\hat{x}_{l}-\hat{v}_{l}\left(\hat{t}_{l}-s\right), v_{l}\right) \mathrm{d} \hat{\Sigma}_{l}(s), \\
& J_{11}+J_{12}+J_{13}=\frac{e^{-\hat{\nu}(v)\left(t-\hat{t}_{1}\right)}}{\tilde{w}(v)} \int_{\Pi_{j=1}^{k-1} \hat{\nu}_{j}} \sum_{l=1}^{k-1} \int_{\hat{t}_{l+1}}^{\hat{t}_{l}} \mathbf{1}_{\left\{\hat{t}_{l+1} \leq 0<\hat{t}_{l}\right\}} \\
& \quad \times\left(1+\left|v_{l}\right|^{2}\right)^{\frac{|\kappa|}{2}}\left[\lambda K_{w}^{m} h^{i-l}+\lambda K_{w}^{c} h^{i-l}+w g\right]\left(\hat{x}_{l}-\hat{v}_{l}\left(\hat{t}_{l}-s\right), v_{l}\right) \mathrm{d} \hat{\Sigma}_{l}(s), \\
& J_{14}=\frac{e^{-\hat{\nu}(v)\left(t-\hat{t}_{1}\right)}}{\tilde{w}(v)} \int_{\Pi_{j=1}^{k-1} \hat{\mathcal{v}}_{j}} I_{\left\{\hat{t}_{k}>0\right\}} h^{i+1-k}\left(\hat{x}_{k}, v_{k-1}\right) \mathrm{d} \hat{\Sigma}_{k-1}\left(\hat{t}_{k}\right),
\end{aligned}
$$

where we have denoted

$$
\mathrm{d} \hat{\Sigma}_{l}(s)=\left\{\Pi_{j=l+1}^{k-1} \mathrm{~d} \hat{\sigma}_{j}\right\} \cdot\left\{\tilde{w}\left(v_{l}\right) e^{-\hat{\nu}\left(v_{l}\right)\left(\hat{t}_{l}-s\right)} \mathrm{d} \hat{\sigma}_{l}\right\} \cdot\left\{\Pi_{j=1}^{l-1} e^{-\hat{\nu}\left(v_{j}\right)\left(\hat{t}_{j}-\hat{t}_{j+1}\right)} \mathrm{d} \hat{\sigma}_{j}\right\} .
$$

Lemma 3.4. Let $\beta>3$. Let $h^{i}, i=0,1,2, \cdots$, be the solutions to (3.9), satisfying

$$
\left\|h^{i}\right\|_{L^{\infty}}+\left|h^{i}\right|_{L^{\infty}(\gamma)}<\infty
$$

Then there exists $T_{0}>0$ large enough such that for $i \geq k:=\hat{C}_{1} T_{0}^{\frac{5}{4}}$, it holds that

$$
\begin{aligned}
\left\|h^{i+1}\right\|_{L^{\infty}}+\left|h^{i+1}\right|_{L^{\infty}(\gamma)} \leq & \frac{1}{8} \sup _{0 \leq l \leq k}\left\{\left\|h^{i-l}\right\|_{L^{\infty}}\right\}+C\left\{\left\|\nu^{-1} w g\right\|_{L^{\infty}}+|w r|_{L^{\infty}\left(\gamma_{-}\right)}\right\} \\
& +C \sup _{0 \leq l \leq k}\left\{\left\|\frac{\sqrt{\nu} h^{i-l}}{w}\right\|_{L^{2}}\right\} .
\end{aligned}
$$

Moreover, if $h^{i} \equiv h$ for $i=1,2, \cdots$, i.e., $h$ is a solution, then (3.11) is reduced to the following estimate

$$
\|h\|_{L^{\infty}}+|h|_{L^{\infty}(\gamma)} \leq C\left\{\left\|\nu^{-1} w g\right\|_{L^{\infty}}+|w r|_{L^{\infty}\left(\gamma_{-}\right)}\right\}+\left\|\frac{\sqrt{\nu} h}{w}\right\|_{L^{2}} .
$$

Here it is emphasized that the positive constant $C>0$ does not depend on $\lambda \in[0,1]$ and $\varepsilon>0$.

Proof. By the definition of $\hat{\nu}(v)$, we first note that

$$
\hat{\nu}(v) \geq\left(1+|v|^{2}\right)^{\frac{|\kappa|}{2}} \nu(v) \geq \hat{\nu}_{0}>0
$$

where $\hat{\nu}_{0}$ is a positive constant independent of $\varepsilon$ and $v \in \mathbb{R}^{3}$. For $J_{1}$, it follows from (3.13) that

$$
\left|J_{1}\right| \leq e^{-\hat{\nu}_{0} t}\left\|h^{i+1}\right\|_{L^{\infty}}
$$


For $J_{2}$, it follows from (2.11) that

$$
\begin{aligned}
\left|J_{2}\right| & \leq C m^{3+\kappa} \int_{\max \left\{\hat{t}_{1}, 0\right\}}^{t} e^{-\hat{\nu}_{0}(t-s)}\left(1+|v|^{2}\right)^{\frac{|\kappa|}{2}} e^{-\frac{|v|^{2}}{20}}\left\|h^{i}\right\| \mathrm{d} s \\
& \leq C m^{3+\kappa} e^{-\frac{|v|^{2}}{32}}\left\|h^{i}\right\|_{L^{\infty}} .
\end{aligned}
$$

For those terms involving the source $g$, we notice that

$$
\frac{1}{\tilde{w}(v)} \leq C w(v) e^{-\frac{|v|^{2}}{4}} \leq C e^{-\frac{|v|^{2}}{8}}
$$

which immediately yields that

$$
\left\{\begin{array}{l}
\int_{\Pi_{j=1}^{k-1} \hat{\mathcal{V}}_{j}} \tilde{w}\left(v_{l}\right)\left[1+\left|v_{l}\right|^{2}\right]^{\frac{|\kappa|}{2}} \Pi_{j=1}^{k-1} \mathrm{~d} \hat{\sigma}_{j} \leq C<\infty, \quad \text { for } \quad 1 \leq l \leq k-1, \\
\int_{\Pi_{j=1}^{k-1} \hat{\mathcal{v}}_{j}} \sum_{l=1}^{k-1} \mathbf{1}_{\left\{\hat{t}_{l+1} \leq 0<\hat{t}_{l}\right\}} \tilde{w}\left(v_{l}\right)\left[1+\left|v_{l}\right|^{2}\right]^{\frac{|\kappa|}{2}} \Pi_{j=1}^{k-1} \mathrm{~d} \hat{\sigma}_{j} \leq C k .
\end{array}\right.
$$

Then it follows from (3.16) and (3.17) that

$$
\begin{gathered}
\left|J_{4}\right|+\left|J_{10}\right|+\left|J_{13}\right| \leq C k\left\|\nu^{-1} w g\right\|_{L^{\infty}}, \\
\left|J_{5}\right|+\left|J_{6}\right| \leq C k|w r|_{L^{\infty}\left(\gamma_{-}\right)}
\end{gathered}
$$

and

$$
\begin{aligned}
\left|J_{7}\right| & \leq C e^{-\frac{1}{8}|v|^{2}} e^{-\hat{\nu}_{0}\left(t-t_{1}\right)} \int_{\Pi_{j=1}^{k-1} \hat{\mathcal{V}}_{j}} \sum_{l=1}^{k-1} \mathbf{1}_{\left\{\hat{t}_{l+1} \leq 0<\hat{t}_{l}\right\}} \mathrm{d} \hat{\Sigma}_{l}(0) \cdot \sup _{1 \leq l \leq k-1}\left\{\left\|h^{i+1-l}\right\|_{L^{\infty}}\right\} \\
& \leq C k e^{-\hat{\nu}_{0} t} e^{-\frac{1}{8}|v|^{2}} \cdot \sup _{1 \leq l \leq k-1}\left\{\left\|h^{i+1-l}\right\|_{L^{\infty}}\right\} .
\end{aligned}
$$

For the term $J_{14}$, it follows from (3.16) and Lemma 3.2 that

$$
\left|J_{14}\right| \leq C e^{-\frac{1}{8}|v|^{2}}\left(\frac{1}{2}\right)^{\hat{C}_{2} T_{0}^{\frac{5}{4}}} \cdot\left|h^{i+1-k}\right|_{L^{\infty}\left(\gamma_{-}\right)},
$$

where we have taken $k=\hat{C}_{1} T_{0}^{\frac{5}{4}}$ and $T_{0}$ is a large constant to be chosen later. From the boundary condition given in the second equation of (3.9), it further holds that

$$
\left|h^{i+1-k}\right|_{L^{\infty}\left(\gamma_{-}\right)} \leq C\left|h^{i-k}\right|_{L^{\infty}\left(\gamma_{+}\right)}+|w r|_{L^{\infty}\left(\gamma_{-}\right)} .
$$

For $J_{8}$, using (2.11), (3.13), (3.16) and (3.17), one obtains that

$$
\begin{aligned}
\left|J_{8}\right| \leq C m^{3+\kappa} e^{-\frac{1}{8}|v|^{2}} \cdot \sup _{1 \leq l \leq k-1}\left\{\left\|h^{i-l}\right\|_{L^{\infty}}\right\} \\
\quad \times \int_{\Pi_{j=1}^{k-1} \hat{\mathcal{v}}_{j}} \sum_{l=1}^{k-1} I_{\left\{\hat{t}_{l+1} \leq 0<\hat{t}_{l}\right\}} \int_{0}^{t_{l}} e^{-\hat{\nu}_{0}(t-s)} \mathrm{d} s \nu\left(v_{l}\right)^{-1} \tilde{w}\left(v_{l}\right) e^{-\frac{\left|v_{l}\right|^{2}}{8}} \Pi_{j=1}^{k-1} \mathrm{~d} \hat{\sigma}_{j} \\
\leq C m^{3+\kappa} e^{-\frac{1}{8}|v|^{2}} \cdot \sup _{1 \leq l \leq k-1}\left\{\left\|h^{i-l}\right\|_{L^{\infty}}\right\} \int_{\Pi_{j=1}^{k-1} \hat{\mathcal{V}}_{j}} \sum_{l=1}^{k-1} I_{\left\{\hat{t}_{l+1} \leq 0<\hat{t}_{l}\right\}} \nu\left(v_{l}\right)^{-1} \tilde{w}\left(v_{l}\right) \Pi_{j=1}^{k-1} \mathrm{~d} \hat{\sigma}_{j} \\
\leq C k m^{3+\kappa} e^{-\frac{1}{8}|v|^{2}} \cdot \sup _{1 \leq l \leq k-1}\left\{\left\|h^{i-l}\right\|_{L^{\infty}}\right\} .
\end{aligned}
$$


Here we remark that the factor $e^{-\frac{1}{8}|v|^{2}}$ on the right-hand side of (3.22) is very crucial for the later use of the Vidav's iteration. For $J_{9}$, it holds that

$$
\begin{aligned}
\left|J_{9}\right| \leq & C e^{-\frac{1}{8}|v|^{2}} \sum_{l=1}^{k-1} \int_{\Pi_{j=1}^{l-1} \hat{\mathcal{V}}_{j}} \mathrm{~d} \hat{\sigma}_{l-1} \cdots \mathrm{d} \hat{\sigma}_{1} \int_{0}^{\hat{t}_{l}} e^{-\hat{\nu}_{0}(t-s)} \mathrm{d} s \\
& \times \int_{\mathcal{V}_{l}} \int_{\mathbb{R}^{3}} \mathbf{1}_{\left\{\hat{t}_{l+1} \leq 0<\hat{t}_{l}\right\}} \nu\left(v_{l}\right)^{-1} \tilde{w}\left(v_{l}\right)\left|k_{w}^{c}\left(v_{l}, v^{\prime}\right) h^{i-l}\left(\hat{x}_{l}-\hat{v}_{l}\left(\hat{t}_{l}-s\right), v^{\prime}\right)\right| \mathrm{d} v^{\prime} \mathrm{d} \hat{\sigma}_{l} \\
= & C e^{-\frac{1}{8}|v|^{2}} \sum_{l=1}^{k-1} \int_{\Pi_{j=1}^{l-1} \hat{\mathcal{V}}_{j}} \mathrm{~d} \hat{\sigma}_{l-1} \cdots \mathrm{d} \hat{\sigma}_{1} \int_{0}^{\hat{t}_{l}} e^{-\hat{\nu}_{0}(t-s)} \mathrm{d} s \int_{\mathcal{V}_{l} \cap\left\{\left|v_{l}\right| \geq N\right\}} \int_{\mathbb{R}^{3}}(\cdots) \mathrm{d} v^{\prime} \mathrm{d} \hat{\sigma}_{l} \\
& +C e^{-\frac{1}{8}|v|^{2}} \sum_{l=1}^{k-1} \int_{\Pi_{j=1}^{l-1} \hat{\mathcal{V}}_{j}} \mathrm{~d} \hat{\sigma}_{l-1} \cdots \mathrm{d} \hat{\sigma}_{1} \int_{0}^{\hat{t}_{l}} e^{-\hat{\nu}_{0}(t-s)} \mathrm{d} s \int_{\mathcal{V}_{l} \cap\left\{\left|v_{l}\right| \leq N\right\}} \int_{\mathbb{R}^{3}}(\cdots) \mathrm{d} v^{\prime} \mathrm{d} \hat{\sigma}_{l} \\
:= & \sum_{l=1}^{k-1}\left(J_{91 l}+J_{92 l}\right) .
\end{aligned}
$$

We shall estimate the right-hand terms of (3.23) as follows. By using (2.14), we have

$$
\begin{aligned}
& \sum_{l=1}^{k-1} J_{91 l} \leq C e^{-\frac{1}{8}|v|^{2}} \sum_{l=1}^{k-1} \int_{\Pi_{j=1}^{l-1} \hat{\mathcal{V}}_{j}} \mathrm{~d} \hat{\sigma}_{l-1} \cdots \mathrm{d} \hat{\sigma}_{1} \int_{0}^{\hat{t}_{l}} e^{-\hat{\nu}_{0}(t-s)} \mathrm{d} s \\
& \times \int_{\mathcal{V}_{l} \cap\left\{\left|v_{l}\right| \geq N\right\}} e^{-\frac{1}{8}\left|v_{l}\right|^{2}} d v_{l} \cdot \sup _{1 \leq l \leq k-1}\left\{\left\|h^{i-l}\right\|_{L^{\infty}}\right\} \\
& \leq C k e^{-\frac{1}{8}|v|^{2}} e^{-\frac{1}{16} N^{2}} \cdot \sup _{1 \leq l \leq k-1}\left\{\left\|h^{i-l}\right\|_{L^{\infty}}\right\} .
\end{aligned}
$$

And, for each term $J_{92 l}$, we also have

$$
\begin{aligned}
J_{92 l} \leq & C e^{-\frac{1}{8}|v|^{2}} \int_{\Pi_{j=1}^{l-1} \hat{\mathcal{V}}_{j}} \mathrm{~d} \hat{\sigma}_{l-1} \cdots \mathrm{d} \hat{\sigma}_{1} \int_{\hat{t}_{l}-\frac{1}{N}}^{\hat{t}_{l}} e^{-\hat{\nu}_{0}(t-s)} \mathrm{d} s \int_{\mathcal{V}_{l} \cap\left\{\left|v_{l}\right| \leq N\right\}} \int_{\mathbb{R}^{3}}(\cdots) \mathrm{d} v^{\prime} \mathrm{d} \hat{\sigma}_{l} \\
& +C e^{-\frac{1}{8}|v|^{2}} \int_{\Pi_{j=1}^{l-1} \hat{\mathcal{V}}_{j}} \mathrm{~d} \hat{\sigma}_{l-1} \cdots \mathrm{d} \hat{\sigma}_{1} \int_{0}^{\hat{t}_{l}-\frac{1}{N}} e^{-\hat{\nu}_{0}(t-s)} \mathrm{d} s \int_{\mathcal{V}_{l} \cap\left\{\left|v_{l}\right| \leq N\right\}} e^{-\frac{1}{8}\left|v_{l}\right|^{2}} \mathrm{~d} v_{l} \\
& \times \int_{\left\{\left|v^{\prime}\right| \geq 2 N\right\}}\left|k_{w}^{c}\left(v_{l}, v^{\prime}\right)\right| e^{\frac{\left|v_{l}-v^{\prime}\right|^{2}}{64}} \mathrm{~d} v^{\prime} e^{-\frac{N^{2}}{64}} \cdot \sup _{1 \leq l \leq k-1}\left\{\left\|h^{i-l}\right\|_{\left.L^{\infty}\right\}}\right. \\
& +C e^{-\frac{1}{8}|v|^{2}} \int_{\Pi_{j=1}^{l-1} \hat{\mathcal{V}}_{j}} \mathrm{~d} \hat{\sigma}_{l-1} \cdots \mathrm{d} \hat{\sigma}_{1} \int_{0}^{\hat{t}_{l}-\frac{1}{N}} e^{-\hat{\nu}_{0}(t-s)} \mathrm{d} s \int_{\mathcal{V}_{l} \cap\left\{\left|v_{l}\right| \leq N\right\}} \int_{\left\{\left|v^{\prime}\right| \leq 2 N\right\}} \\
& \times \mathbf{1}_{\left\{\hat{t}_{l+1} \leq 0<\hat{t}_{l}\right\}} e^{-\frac{1}{8}\left|v_{l}\right|^{2}}\left|k_{w}^{c}\left(v_{l}, v^{\prime}\right) h^{i-l}\left(x_{l}-\hat{v}_{l}\left(\hat{t}_{l}-s\right), v^{\prime}\right)\right| \mathrm{d} v^{\prime} \mathrm{d} v_{l} \\
\leq & C e^{-\frac{1}{8}|v|^{2}} \int_{\Pi_{j=1}^{l-1} \hat{\nu}_{j}} \mathrm{~d} \hat{\sigma}_{l-1} \cdots \mathrm{d} \hat{\sigma}_{1} \int_{0}^{\hat{t}_{l}-\frac{1}{N}} e^{-\hat{\nu}_{0}(t-s)} \mathrm{d} s \int_{\mathcal{V}_{l} \cap\left\{\left|v_{l}\right| \leq N\right\}} \int_{\left|v^{\prime}\right| \leq 2 N} \\
& \times \mathbf{1}_{\left\{\hat{t}_{l+1} \leq 0<\hat{t}_{l}\right\}} e^{-\frac{1}{8}\left|v_{l}\right|^{2}\left|k_{w}^{c}\left(v_{l}, v^{\prime}\right) h^{i-l}\left(x_{l}-\hat{v}_{l}\left(\hat{t}_{l}-s\right), v^{\prime}\right)\right| \mathrm{d} v^{\prime} \mathrm{d} v_{l}} \\
& +\frac{C}{N} e^{-\frac{1}{8}|v|^{2} \cdot\left\|h^{i-l}\right\|_{L^{\infty}} .}
\end{aligned}
$$


To estimate the first term on the right-hand side of (3.25), it follows from (2.12) that

$$
\begin{aligned}
& \int_{\mathcal{V}_{l} \cap\left\{\left|v_{l}\right| \leq N\right\}} \int_{\left|v^{\prime}\right| \leq 2 N} \mathbf{1}_{\left\{\hat{t}_{l+1} \leq 0<\hat{t}_{l}\right\}} e^{-\frac{1}{8}\left|v_{l}\right|^{2}}\left|k_{w}^{c}\left(v_{l}, v^{\prime}\right) h^{i-l}\left(\hat{x}_{l}-\hat{v}_{l}\left(\hat{t}_{l}-s\right), v^{\prime}\right)\right| \mathrm{d} v^{\prime} \mathrm{d} v_{l} \\
& \leq C_{N}\left\{\int_{\mathcal{V}_{l} \cap\left\{\left|v_{l}\right| \leq N\right\}} \int_{\left|v^{\prime}\right| \leq 2 N} e^{-\frac{1}{8}\left|v_{l}\right|^{2}}\left|k_{w}^{c}\left(v_{l}, v^{\prime}\right)\right|^{2} \mathrm{~d} v^{\prime} \mathrm{d} v_{l}\right\}^{\frac{1}{2}} \\
& \quad \times\left\{\int_{\mathcal{V}_{l} \cap\left\{\left|v_{l}\right| \leq N\right\}} \int_{\left|v^{\prime}\right| \leq 2 N} \mathbf{1}_{\left\{\hat{t}_{l+1} \leq 0<\hat{t}_{l}\right\}}\left|\frac{\sqrt{\nu\left(v^{\prime}\right)} h^{i-l}\left(\hat{x}_{l}-\hat{v}_{l}\left(\hat{t}_{l}-s\right), v^{\prime}\right)}{w\left(v^{\prime}\right)}\right|^{2} \mathrm{~d} v^{\prime} \mathrm{d} v_{l}\right\}^{\frac{1}{2}} \\
& \leq C_{N} m^{\kappa-1}\left\{\int_{\mathcal{V}_{l} \cap\left\{\left|v_{l}\right| \leq N\right\}} \int_{\left|v^{\prime}\right| \leq 2 N} \mathbf{1}_{\left\{\hat{t}_{l+1} \leq 0<\hat{t}_{l}\right\}}\left|\frac{\sqrt{\nu\left(v^{\prime}\right)} h^{i-l}\left(\hat{x}_{l}-\hat{v}_{l}\left(\hat{t}_{l}-s\right), v^{\prime}\right)}{w\left(v^{\prime}\right)}\right|^{2} \mathrm{~d} v^{\prime} \mathrm{d} v_{l}\right\}^{\frac{1}{2}} .
\end{aligned}
$$

Let $y_{l}=\hat{x}_{l}-\hat{v}\left(\hat{t}_{l}-s\right) \in \Omega$ for $s \in\left[0, \hat{t}_{l}-\frac{1}{N}\right]$. A direct computation shows that

$$
\left|\frac{\partial y_{l}}{\partial v_{l}}\right|=\left|\hat{t}_{l}-s\right| \cdot\left|\frac{\partial \hat{v}}{\partial v}\right| \geq \frac{\left(1+|v|^{2}\right)^{\frac{3|\kappa|}{2}-1}}{N^{3}} \cdot\left\{1+(1+|\kappa|)|v|^{2}\right\} \geq \frac{1}{N^{3}} .
$$

Thus, by making change of variable $\hat{v}_{l} \rightarrow y_{l}$ and using (3.27), one obtains that

$$
\begin{aligned}
& \left\{\int_{\mathcal{V}_{l} \cap\left\{\left|v_{l}\right| \leq N\right\}} \int_{\left|v^{\prime}\right| \leq 2 N} \mathbf{1}_{\left\{\hat{t}_{l+1} \leq 0<\hat{t}_{l}\right\}}\left|\frac{\sqrt{\nu\left(v^{\prime}\right)} h^{i-l}\left(\hat{x}_{l}-\hat{v}_{l}\left(\hat{t}_{l}-s\right), v^{\prime}\right)}{w\left(v^{\prime}\right)}\right|^{2} \mathrm{~d} v^{\prime} \mathrm{d} v_{l}\right\}^{\frac{1}{2}} \\
& \leq C_{N}\left\{\int_{\Omega} \int_{\left|v^{\prime}\right| \leq 2 N}\left|\frac{\sqrt{\nu\left(v^{\prime}\right)} h^{i-l}\left(y_{l}, v^{\prime}\right)}{w\left(v^{\prime}\right)}\right|^{2} \mathrm{~d} v^{\prime} \mathrm{d} y_{l}\right\}^{\frac{1}{2}} \leq C_{N}\left\|\frac{\sqrt{\nu} h^{i-l}}{w}\right\|_{L^{2}},
\end{aligned}
$$

which together with (3.26) and (3.25) yield that

$$
J_{92 l} \leq \frac{C}{N} e^{-\frac{1}{8}|v|^{2}} \cdot \sup _{1 \leq l \leq k-1}\left\{\left\|h^{i-l}\right\|_{L^{\infty}}\right\}+C_{N} m^{\kappa-1} e^{-\frac{1}{8}|v|^{2}}\left\|\frac{\sqrt{\nu} h^{i-l}}{w}\right\|_{L^{2}} .
$$

Thus it follows from (3.28), (3.24) and (3.23) that

$$
\left|J_{9}\right| \leq \frac{C k}{N} e^{-\frac{1}{8}|v|^{2}} \cdot \sup _{1 \leq l \leq k-1}\left\{\left\|h^{i-l}\right\|_{L^{\infty}}\right\}+C_{N} k m^{\kappa-1} e^{-\frac{1}{8}|v|^{2}} . \sup _{1 \leq l \leq k-1}\left\{\left\|\frac{\sqrt{\nu} h^{i-l}}{w}\right\|_{L^{2}}\right\} .
$$

By similar arguments as in (3.22)-(3.29), one can obtain

$$
\begin{array}{rl}
\left|J_{11}\right|+\left|J_{12}\right| \leq C & C e^{-\frac{1}{8}|v|^{2}}\left\{m^{3+\kappa}+\frac{1}{N}\right\} \cdot \sup _{1 \leq l \leq k-1}\left\{\left\|h^{i-l}\right\|_{L^{\infty}}\right\} \\
+ & C_{N} k m^{\kappa-1} e^{-\frac{1}{8}|v|^{2}} \cdot \sup _{1 \leq l \leq k-1}\left\{\left\|\frac{\sqrt{\nu} h^{i-l}}{w}\right\|_{L^{2}}\right\} .
\end{array}
$$

Now substituting (3.30), (3.29), (3.22), (3.21), (3.20), (3.19), (3.18), (3.15) and (3.14) into (3.10), we get, for $t \in\left[0, T_{0}\right]$, that

$$
\begin{aligned}
\left|h^{i+1}(x, v)\right| \leq & \int_{\max \left\{\hat{t}_{1}, 0\right\}}^{t} e^{-\hat{\nu}_{0}(t-s)}\left(1+|v|^{2}\right)^{\frac{|\kappa|}{2}} \int_{\mathbb{R}^{3}}\left|k_{w}^{c}\left(v, v^{\prime}\right) h^{i}\left(x-\hat{v}(t-s), v^{\prime}\right)\right| \mathrm{d} v^{\prime} \mathrm{d} s \\
& +A_{i}(t, v)
\end{aligned}
$$


where we have denoted

$$
\begin{aligned}
A_{i}(t, v):= & C k e^{-\frac{1}{32}|v|^{2}}\left\{m^{3+\kappa}+e^{-\hat{\nu}_{0} t}+\left(\frac{1}{2}\right)^{\hat{C}_{2} T_{0}^{\frac{5}{4}}}+\frac{1}{N}\right\} \cdot \sup _{0 \leq l \leq k-1}\left\{\left\|h^{i-l}\right\|_{\left.L^{\infty}+\left|h^{i-l}\right|_{L^{\infty}\left(\gamma_{+}\right)}\right\}}\right. \\
& +e^{-\hat{\nu}_{0} t}\left\|h^{i+1}\right\|_{L^{\infty}}+C k\left\{\left\|\nu^{-1} w g\right\|_{L^{\infty}}+|w r|_{L^{\infty}\left(\gamma_{-}\right)}\right\} \\
& +C_{N, k, m} e^{-\frac{1}{8}|v|^{2}} \cdot \sup _{1 \leq l \leq k-1}\left\{\left\|\frac{\sqrt{\nu} h^{i-l}}{w}\right\|_{L^{2}}\right\} .
\end{aligned}
$$

We denote $x^{\prime}=x-\hat{v}(t-s) \in \Omega$ and $\hat{t}_{1}^{\prime}=\hat{t}_{1}\left(s, x^{\prime}, v^{\prime}\right)$ for $s \in\left(\min \left\{t_{1}, 0\right\}, t\right)$. Using the Vidav's iteration in (3.31), then we obtain that

$$
\begin{aligned}
\left|h^{i+1}(x, v)\right| \leq & A_{i}(t, v)+\int_{0}^{t} e^{-\hat{\nu}_{0}(t-s)}\left(1+|v|^{2}\right)^{\frac{|\kappa|}{2}} \int_{\mathbb{R}^{3}}\left|k_{w}^{c}\left(v, v^{\prime}\right)\right| A_{i-1}\left(s, v^{\prime}\right) \mathrm{d} v^{\prime} \mathrm{d} s \\
& +\int_{0}^{t} d s \int_{0}^{s} e^{-\hat{\nu}_{0}(t-\tau)} \mathrm{d} \tau \int_{\mathbb{R}^{3}} \int_{\mathbb{R}^{3}}\left|k_{w}^{c}\left(v, v^{\prime}\right) k_{w}^{c}\left(v^{\prime}, v^{\prime \prime}\right)\right| \\
& \times \mathbf{1}_{\left\{\max \left\{\hat{t}_{1}, 0\right\}<s<t\right\}} \mathbf{1}_{\left\{\max \left\{\hat{t}_{1}^{\prime}, 0\right\}<\tau<s\right\}}\left|h^{i-1}\left(x^{\prime}-\hat{v}^{\prime}(s-\tau), v^{\prime \prime}\right)\right| \mathrm{d} v^{\prime \prime} \mathrm{d} v^{\prime} \\
:= & A_{i}(t, v)+B_{1}+B_{2} .
\end{aligned}
$$

For the term $B_{1}$, using (2.13) and (2.14), one has

$$
\begin{aligned}
B_{1} \leq & C k\left\{m^{3+\kappa}+m^{\kappa-1} e^{-\frac{1}{2} \hat{\nu}_{0} t}+\left(\frac{1}{2}\right)^{\hat{C}_{2} T_{0}^{\frac{5}{4}}}+\frac{1}{N}\right\} \cdot \sup _{0 \leq l \leq k}\left\{\left\|h^{i-l}\right\|_{L^{\infty}}+\left|h^{i-l}\right|_{L^{\infty}\left(\gamma_{+}\right)}\right\} \\
& +C_{k, m}\left\{\left\|\nu^{-1} w g\right\|_{L^{\infty}}+|w r|_{L^{\infty}\left(\gamma_{-}\right)}\right\}+C_{N, k, m} \sup _{0 \leq l \leq k}\left\{\left\|\frac{\sqrt{\nu} h^{i-l}}{w}\right\|_{L^{2}}\right\} .
\end{aligned}
$$

For the term $B_{2}$, we split the estimate by several cases.

Case 1. For $|v| \geq N$, we have from (2.13) that

$$
B_{2} \leq C m^{2(\kappa-1)}\left\|h^{i-1}\right\|_{L^{\infty}}(1+|v|)^{-4} \leq \frac{C m^{2(\kappa-1)}}{N^{4}}\left\|h^{i-1}\right\|_{L^{\infty}} .
$$

Case 2. For $|v| \leq N,\left|v^{\prime}\right| \geq 2 N$ or $\left|v^{\prime}\right| \leq 2 N,\left|v^{\prime \prime}\right| \geq 3 N$. In this case, we note from (2.13) that

$$
\left\{\begin{array}{l}
\int_{|v| \leq N,\left|v^{\prime}\right| \geq 2 N}\left|k_{w}^{c}\left(v, v^{\prime}\right) e^{\frac{\left|v-v^{\prime}\right|^{2}}{32}}\right| \mathrm{d} v^{\prime} \leq C m^{\kappa-1}(1+|v|)^{\kappa-2}, \\
\int_{\left|v^{\prime}\right| \leq 2 N,\left|v^{\prime \prime}\right| \geq 3 N}\left|k_{w}^{c}\left(v^{\prime}, v^{\prime \prime}\right) e^{\frac{\left|v^{\prime}-v^{\prime \prime}\right|^{2}}{32}}\right| \mathrm{d} v^{\prime \prime} \leq C m^{\kappa-1}\left(1+\left|v^{\prime}\right|\right)^{\kappa-2} .
\end{array}\right.
$$

This yields that

$$
\begin{aligned}
& \int_{0}^{t} \mathrm{~d} s \int_{0}^{s} e^{-\hat{\nu}_{0}(t-\tau)} \mathrm{d} \tau\left\{\int_{|v| \leq N,\left|v^{\prime}\right| \geq 2 N}+\int_{\left|v^{\prime}\right| \leq 2 N,\left|v^{\prime \prime}\right| \geq 3 N}\right\}(\cdots) \mathrm{d} v^{\prime \prime} \mathrm{d} v^{\prime} \\
& \leq e^{-\frac{N^{2}}{32}}\left\|h^{i-1}\right\|_{L^{\infty}} \int_{|v| \leq N,\left|v^{\prime}\right| \geq 2 N}\left|k_{w}^{c}\left(v, v^{\prime}\right) e^{\frac{\left.\left|v-v^{\prime}\right|\right|^{2}}{32}}\right| \cdot\left|k_{w}^{c}\left(v^{\prime}, v^{\prime \prime}\right)\right| \nu(v)^{-1} \nu\left(v^{\prime}\right)^{-1} \mathrm{~d} v^{\prime \prime} \mathrm{d} v^{\prime} \\
& \quad+e^{-\frac{N^{2}}{32}}\left\|h^{i-1}\right\|_{L^{\infty}} \int_{|v| \leq N,\left|v^{\prime}\right| \geq 2 N}\left|k_{w}^{c}\left(v, v^{\prime}\right)\right| \cdot\left|k_{w}^{c}\left(v^{\prime}, v^{\prime \prime}\right) e^{\frac{\left|v^{\prime}-v^{\prime \prime}\right|^{2}}{32}}\right| \nu(v)^{-1} \nu\left(v^{\prime}\right)^{-1} \mathrm{~d} v^{\prime \prime} \mathrm{d} v^{\prime} \\
& \leq C m^{2(\kappa-1)} e^{-\frac{N^{2}}{32}}\left\|h^{i-1}\right\|_{L^{\infty}} .
\end{aligned}
$$


Case 3. For $|v| \leq N,\left|v^{\prime}\right| \leq 2 N$, and $\left|v^{\prime \prime}\right| \leq 3 N$, we first note that

$$
\begin{aligned}
& \int_{0}^{t} \mathrm{~d} s \int_{0}^{s} e^{-\hat{\nu}_{0}(t-\tau)} \mathrm{d} \tau \int_{\left|v^{\prime}\right| \leq 2 N,\left|v^{\prime \prime}\right| \leq 3 N}(\cdots) \mathrm{d} v^{\prime \prime} \mathrm{d} v^{\prime} \\
& \leq \frac{C}{N} m^{2(\kappa-1)}\left\|h^{i-1}\right\|_{L^{\infty}}+\int_{0}^{t} \mathrm{~d} s \int_{0}^{s-\frac{1}{N}} e^{-\hat{\nu}_{0}(t-\tau)} \mathrm{d} \tau \int_{\mathbb{R}^{3}} \int_{\mathbb{R}^{3}}(\cdots) \mathrm{d} v^{\prime \prime} \mathrm{d} v^{\prime} \\
& \leq \frac{C}{N} m^{2(\kappa-1)}\left\|h^{i-1}\right\|_{L^{\infty}} \\
& +C_{N, k} \int_{0}^{t} \mathrm{~d} s \int_{0}^{s-\frac{1}{N}} e^{-\hat{\nu}_{0}(t-\tau)} \mathrm{d} \tau\left\{\int_{\left|v^{\prime}\right| \leq 2 N,\left|v^{\prime \prime}\right| \leq 3 N}\left|k^{c}\left(v, v^{\prime}\right) k^{c}\left(v^{\prime}, v^{\prime \prime}\right)\right|^{2} \mathrm{~d} v^{\prime \prime} \mathrm{d} v^{\prime}\right\}^{\frac{1}{2}} \\
& \quad \times\left\{\int_{\left|v^{\prime}\right| \leq 2 N,\left|v^{\prime \prime}\right| \leq 3 N} \mathbf{1}_{\left\{\max \left\{\hat{t}_{1}, 0\right\}<s<t\right\}} \mathbf{1}_{\left\{\max \left\{\hat{t}_{1}^{\prime}, 0\right\}<\tau<s\right\}}\left|\frac{\sqrt{\nu\left(v^{\prime \prime}\right)} h^{i-1}\left(y^{\prime}, v^{\prime \prime}\right)}{w\left(v^{\prime \prime}\right)}\right|^{2} \mathrm{~d} v^{\prime \prime} \mathrm{d} v^{\prime}\right\}^{\frac{1}{2}} \\
& \leq \frac{C}{N} m^{2(\kappa-1)}\left\|h^{i-1}\right\|_{L^{\infty}}+C_{N, k, m} \int_{0}^{t} \mathrm{~d} s \int_{0}^{s-\frac{1}{N}} e^{-\hat{\nu}_{0}(t-\tau)} \mathrm{d} \tau \\
& \quad \times\left\{\int_{\left|v^{\prime}\right| \leq 2 N,\left|v^{\prime \prime}\right| \leq 3 N} \mathbf{1}_{\left\{\max \left\{\hat{t}_{1}, 0\right\}<s<t\right\}} \mathbf{1}_{\left\{\max \left\{\hat{t}_{1}^{\prime}, 0\right\}<\tau<s\right\} \mid}\left|\frac{\sqrt{\nu\left(v^{\prime \prime}\right)} h^{i-1}\left(y^{\prime}, v^{\prime \prime}\right)}{w\left(v^{\prime \prime}\right)}\right|^{2} \mathrm{~d} v^{\prime \prime} \mathrm{d} v^{\prime}\right\}^{\frac{1}{2}},
\end{aligned}
$$

where we have denoted $y^{\prime}=x^{\prime}-\hat{v}^{\prime}(s-\tau) \in \Omega$ for $s \in\left(\max \left\{\hat{t}_{1}, 0\right\}, s\right)$ and $\tau \in\left(\max \left\{\hat{t}_{1}^{\prime}, 0\right\}, s\right)$. Similar to (3.27), we make change of variable $v^{\prime} \mapsto y^{\prime}$, so that the second term on the right-hand side of (3.36) is bounded as

$$
\int_{0}^{t} \mathrm{~d} s \int_{0}^{s-\frac{1}{N}} e^{-\hat{\nu}_{0}(t-\tau)} \mathrm{d} \tau\left\{\int_{\left|v^{\prime}\right| \leq 2 N,\left|v^{\prime \prime}\right| \leq 3 N}(\cdots) \mathrm{d} v^{\prime \prime} \mathrm{d} v^{\prime}\right\}^{\frac{1}{2}} \leq C N^{\frac{3}{2}}\left\|\frac{\sqrt{\nu} h^{i-1}}{w}\right\|_{L^{2}},
$$

which together with (3.36) yield that

$$
\begin{aligned}
\int_{0}^{t} \mathrm{~d} s \int_{0}^{s} e^{-\hat{\nu}_{0}(t-\tau)} \mathrm{d} \tau \int_{\left|v^{\prime}\right| \leq 2 N,\left|v^{\prime \prime}\right| \leq 3 N}(\cdots) \mathrm{d} v^{\prime \prime} \mathrm{d} v^{\prime} & \\
& \leq \frac{C}{N} m^{2(\kappa-1)}\left\|h^{i-1}\right\|_{L^{\infty}}+C_{N, k, m}\left\|\frac{\sqrt{\nu} h^{i-1}}{w}\right\|_{L^{2}} .
\end{aligned}
$$

Combining (3.34), (3.35) and (3.37), we have

$$
B_{2} \leq \frac{C}{N} m^{2(\kappa-1)}\left\|h^{i-1}\right\|_{L^{\infty}}+C_{N, k, m}\left\|\frac{\sqrt{\nu} h^{i-1}}{w}\right\|_{L^{2}} .
$$

Hence, the above estimate together with (3.33) and (3.32) yields that for any $t \in\left[0, T_{0}\right]$,

$$
\begin{aligned}
\left|h^{i+1}(x, v)\right| \leq & C k\left\{m^{3+\kappa}+m^{\kappa-1} e^{-\frac{1}{2} \hat{\nu}_{0} t}+\left(\frac{1}{2}\right)^{\hat{C}_{2} T_{0}^{\frac{5}{4}}}+\frac{m^{2(\kappa-1)}}{N}\right\} \\
& \times \sup _{0 \leq l \leq k}\left\{\left\|h^{i-l}\right\|_{\left.L^{\infty}+\left|h^{i-l}\right|_{L^{\infty}\left(\gamma_{+}\right)}\right\}}\right. \\
& +e^{-\hat{\nu}_{0} t}\left\|h^{i+1}\right\|_{L^{\infty}}+C_{k, m}\left\{\left\|\nu^{-1} w g\right\|_{L^{\infty}}+|w r|_{L^{\infty}\left(\gamma_{-}\right)}\right\} \\
& +C_{N, k, m} \sup _{0 \leq l \leq k}\left\{\left\|\frac{\sqrt{\nu} h^{i-l}}{w}\right\|_{L^{2}}\right\} .
\end{aligned}
$$


Now we take $k=\hat{C}_{1} t^{\frac{5}{4}}=\hat{C}_{1} T_{0}^{\frac{5}{4}}$ and choose $m=T_{0}^{-\frac{9}{4(3+\kappa)}}$. We first fix $t=T_{0}$ large enough, and then choose $N$ large enough, so that one has $e^{-\hat{\nu}_{0} t} \leq \frac{1}{2}$ and

$$
C k\left\{m^{3+\kappa}+m^{\kappa-1} e^{-\frac{1}{2} \hat{\nu}_{0} t}+\left(\frac{1}{2}\right)^{\hat{C}_{2} T_{0}^{\frac{5}{4}}}+\frac{m^{2(\kappa-1)}}{N}\right\} \leq \frac{1}{16} .
$$

Therefore (3.11) follows. This completes the proof of Lemma 3.4

3.2. Approximate sequence. Now we are in a position to construct solutions to (3.1) or equivalently (1.7). First of all, we consider the following approximate problem

$$
\left\{\begin{array}{l}
\varepsilon f^{n}+v \cdot \nabla_{x} f^{n}+\nu(v) f^{n}-K f^{n}=g, \\
\left.f^{n}(x, v)\right|_{\gamma_{-}}=\left(1-\frac{1}{n}\right) P_{\gamma} f^{n}+r
\end{array}\right.
$$

where $\varepsilon \in(0,1]$ is arbitrary and $n>1$ is an integer. Recall $k=\hat{C}_{1} T_{0}^{\frac{5}{4}}$ with $T_{0}$ large enough. To the end, we choose $n_{0}>1$ large enough such that

$$
\frac{1}{8}\left(1-\frac{2}{n}+\frac{3}{2 n^{2}}\right)^{-\frac{k+1}{2}} \leq \frac{1}{2}
$$

for any $n \geq n_{0}$.

Lemma 3.5. Let $\varepsilon>0, n \geq n_{0}$, and $\beta>3$. Assume $\left\|\nu^{-1} w g\right\|_{L^{\infty}}+|w r|_{L^{\infty}\left(\gamma_{-}\right)}<\infty$. Then there exists a unique solution $f^{n}$ to (3.38) satisfying

$$
\left\|w f^{n}\right\|_{L^{\infty}}+\left|w f^{n}\right|_{L^{\infty}(\gamma)} \leq C_{\varepsilon, n}\left(|w r|_{L^{\infty}\left(\gamma_{-}\right)}+\left\|\nu^{-1} w g\right\|_{L^{\infty}}\right)
$$

where the positive constant $C_{\varepsilon, n}>0$ depends only on $\varepsilon$ and $n$. Moreover, if $\Omega$ is a strictly convex domain, $g$ is continuous in $\Omega \times \mathbb{R}^{3}$ and $r$ is continuous in $\gamma_{-}$, then $f^{n}$ is continuous away from grazing set $\gamma_{0}$.

Proof. We consider the solvability of the following boundary value problem

$$
\left\{\begin{array}{l}
\mathcal{L}_{\lambda} f:=\varepsilon f+v \cdot \nabla_{x} f+\nu(v) f-\lambda K f=g, \\
\left.f(x, v)\right|_{\gamma_{-}}=\left(1-\frac{1}{n}\right) P_{\gamma} f+r(x, v),
\end{array}\right.
$$

for $\lambda \in[0,1]$. For brevity we denote $\mathcal{L}_{\lambda}^{-1}$ to be the solution operator associated with the problem, meaning that $f:=\mathcal{L}_{\lambda}^{-1} g$ is a solution to the BVP (3.39). Our idea is to prove the existence of $\mathcal{L}_{0}^{-1}$, and then extend to obtain the existence of $\mathcal{L}_{1}^{-1}$ in a continuous argument on $\lambda$. Since the proof is very long, we split it into several steps.

Step 1. In this step, we prove the existence of $\mathcal{L}_{0}^{-1}$. We consider the following approximate sequence

$$
\left\{\begin{array}{l}
\mathcal{L}_{0} f^{i+1}=\varepsilon f^{i+1}+v \cdot \nabla_{x} f^{i+1}+\nu(v) f^{i+1}=g, \\
\left.f^{i+1}(x, v)\right|_{\gamma_{-}}=\left(1-\frac{1}{n}\right) P_{\gamma} f^{i}+r
\end{array}\right.
$$

for $i=0,1,2, \cdots$, where we have set $f^{0} \equiv 0$. We will construct $L^{\infty}$ solutions to (3.40) for $i=0,1,2, \cdots$, and establish uniform $L^{\infty}$-estimates.

Firstly, we will solve inductively the linear equation (3.40) by the method of characteristics. Let $h^{i+1}(x, v)=w(v) f^{i+1}(x, v)$. For almost every $(x, v) \in \bar{\Omega} \times \mathbb{R}^{3} \backslash\left(\gamma_{0} \cup \gamma_{-}\right)$, one can write

$$
\begin{aligned}
h^{i+1}(x, v)= & e^{-(\varepsilon+\nu(v)) t_{\mathbf{b}}} w(v)\left[\left(1-\frac{1}{n}\right) P_{\gamma} f^{i}+r\right]\left(x_{\mathbf{b}}(x, v), v\right) \\
& +\int_{t_{1}}^{t} e^{-(\varepsilon+\nu(v))(t-s)}(w g)(x-v(t-s), v) \mathrm{d} s
\end{aligned}
$$


and for $(x, v) \in \gamma_{-}$, we write

$$
h^{i+1}(x, v)=w(v)\left[\left(1-\frac{1}{n}\right) P_{\gamma} f^{i}+r\right](x, v) .
$$

Noting the definition of $P_{\gamma} f$, we have

$$
\left|w P_{\gamma} f\right|_{L^{\infty}} \leq C|f|_{L^{\infty}\left(\gamma_{+}\right)} .
$$

We consider (3.41) with $i=0$. Noting $h^{0} \equiv 0$, then it is straightforward to see that

$$
\left\|h^{1}\right\|_{L^{\infty}} \leq|w r|_{L^{\infty}\left(\gamma_{-}\right)}+\frac{C}{\varepsilon}\|w g\|_{L^{\infty}}<\infty .
$$

Therefore we have obtained the solution to (3.40) with $i=0$. Assume that we have already solved (3.40) for $i \leq l$ and obtained

$$
\left\|h^{l+1}\right\|_{L^{\infty}}+\left|h^{l+1}\right|_{L^{\infty}(\gamma)} \leq C_{\varepsilon, n, l+1}\left(|w r|_{L^{\infty}\left(\gamma_{-}\right)}+\|w g\|_{L^{\infty}}\right)<\infty .
$$

We now consider (3.40) for $i=l+1$. Noting (3.44), then we can solve (3.40) by using (3.41) and (3.42) with $i=l+1$. We still need to prove $h^{l+2} \in L^{\infty}$. Indeed, it follows from (3.41), (3.42) and (3.43) that

$$
\begin{aligned}
\left\|h^{l+2}\right\|_{L^{\infty}}+\left|h^{l+2}\right|_{L^{\infty}(\gamma)} & \leq C\left(|w r|_{L^{\infty}\left(\gamma_{-}\right)}+\left|h^{l+1}\right|_{L^{\infty}\left(\gamma_{+}\right)}\right)+\frac{C}{\varepsilon}\|w g\|_{L^{\infty}} \\
& \leq C_{\varepsilon, n, l+2}\left(|w r|_{L^{\infty}\left(\gamma_{-}\right)}+\|w g\|_{L^{\infty}}\right)<\infty .
\end{aligned}
$$

Therefore, inductively we have solved (3.40) for $i=0,1,2, \cdots$ and obtained

$$
\left\|h^{i}\right\|_{L^{\infty}}+\left|h^{i}\right|_{L^{\infty}(\gamma)} \leq C_{\varepsilon, n, i}\left(|w r|_{L^{\infty}(\gamma-)}+\|w g\|_{L^{\infty}}\right)<\infty,
$$

for $i=0,1,2, \cdots$. The positive constant $C_{\varepsilon, n, i}$ may increase to infinity as $i \rightarrow \infty$. Here, we emphasize that we first need to know the sequence $\left\{h^{i}\right\}_{i=0}^{\infty}$ is in $L^{\infty}$-space, otherwise one can not use Lemma 3.4 to get uniform $L^{\infty}$ estimates.

If $\Omega$ is a convex domain, let $(x, v) \in \Omega \times \mathbb{R}^{3} \backslash \gamma_{0}$, then it holds $v \cdot n\left(x_{\mathbf{b}}(x, v)\right)<0$ which yields that $t_{\mathbf{b}}(x, v)$ and $x_{\mathbf{b}}(x, v)$ are smooth by Lemma 2 in [29]. Therefore if $g$ and $r$ are continuous, we have that $f^{i}(x, v)$ is continuous away from grazing set.

Secondly, in order to take the limit $i \rightarrow \infty$, one has to get some uniform estimates. Multiplying (3.40) by $f^{i+1}$ and integrating the resultant equality over $\Omega \times \mathbb{R}^{3}$, one obtains that

$$
\begin{aligned}
& \varepsilon\left\|f^{i+1}\right\|_{L^{2}}^{2}+\frac{1}{2}\left|f^{i+1}\right|_{L^{2}\left(\gamma_{+}\right)}^{2}+\left\|f^{i+1}\right\|_{\nu}^{2} \\
& \leq \frac{1}{2}\left(1-\frac{2}{n}+\frac{3}{2 n^{2}}\right)\left|f^{i}\right|_{L^{2}\left(\gamma_{+}\right)}^{2}+C_{n}|r|_{L^{2}\left(\gamma_{-}\right)}^{2}+\frac{C}{\varepsilon}\|g\|_{L^{2}}^{2}+\frac{\varepsilon}{4}\left\|f^{i+1}\right\|_{L^{2}}^{2},
\end{aligned}
$$

where we have used $\left|P_{\gamma} f^{i}\right|_{L^{2}\left(\gamma_{-}\right)}=\left|P_{\gamma} f^{i}\right|_{L^{2}\left(\gamma_{+}\right)} \leq\left|f^{i}\right|_{L^{2}\left(\gamma_{+}\right)}$. Then, from (3.46), we have

$$
\begin{aligned}
& \frac{3}{2} \varepsilon\left\|f^{i+1}\right\|_{L^{2}}^{2}+\left|f^{i+1}\right|_{L^{2}\left(\gamma_{+}\right)}^{2}+2\left\|f^{i+1}\right\|_{\nu}^{2} \\
& \leq\left(1-\frac{2}{n}+\frac{3}{2 n^{2}}\right)\left|f^{i}\right|_{L^{2}\left(\gamma_{+}\right)}^{2}+C_{\varepsilon, n}\left\{|r|_{L^{2}\left(\gamma_{-}\right)}^{2}+\|g\|_{L^{2}}^{2}\right\} .
\end{aligned}
$$


Now we take the difference $f^{i+1}-f^{i}$ in (3.40), then by similar energy estimate as above, we obtain

$$
\begin{aligned}
& \frac{3}{2} \varepsilon\left\|f^{i+1}-f^{i}\right\|_{L^{2}}^{2}+\left|f^{i+1}-f^{i}\right|_{L^{2}\left(\gamma_{+}\right)}^{2}+2\left\|f^{i+1}-f^{i}\right\|_{\nu}^{2} \\
& \leq\left(1-\frac{2}{n}+\frac{3}{2 n^{2}}\right)\left|f^{i}-f^{i-1}\right|_{L^{2}\left(\gamma_{+}\right)}^{2} \\
& \leq \cdots \\
& \leq\left(1-\frac{2}{n}+\frac{3}{2 n^{2}}\right)^{i}\left|f^{1}\right|_{L^{2}(\gamma)}^{2} \\
& \leq C_{\varepsilon} \cdot\left(1-\frac{2}{n}+\frac{3}{2 n^{2}}\right)^{i} \cdot\left(|w r|_{L^{\infty}\left(\gamma_{-}\right)}+\|w g\|_{L^{\infty}}\right)<\infty .
\end{aligned}
$$

Noting $1-\frac{2}{n}+\frac{3}{2 n^{2}}<1$, thus $\left\{f^{i}\right\}_{i=0}^{\infty}$ is a Cauchy sequence in $L^{2}$, i.e.,

$$
\left\|f^{i}-f^{j}\right\|_{L^{2}}^{2}+\left|f^{i}-f^{j}\right|_{L^{2}\left(\gamma_{+}\right)}^{2}+\left\|f^{i}-f^{j}\right\|_{\nu}^{2} \rightarrow 0, \quad \text { as } i, j \rightarrow \infty .
$$

And we also have, for $i=0,1,2, \cdots$, that

$$
\left\|f^{i}\right\|_{L^{2}}^{2}+\left|f^{i}\right|_{L^{2}\left(\gamma_{+}\right)}^{2}+\left\|f^{i}\right\|_{\nu}^{2} \leq C_{\varepsilon, n}\left\{|r|_{L^{2}\left(\gamma_{-}\right)}^{2}+\|g\|_{L^{2}}^{2}\right\},
$$

where $C_{\varepsilon, n}>0$ is a positive constant which depends only on $\varepsilon$ and $n$.

Next we consider the uniform $L^{\infty}$ estimate. Here we point out that Lemma 3.4 still holds by replacing 1 with $1-\frac{1}{n}$ in the boundary condition, and the constants in Lemma 3.4 do not depend on $n \geq 1$. Thus we apply Lemma 3.4 to obtain that

$$
\begin{aligned}
\left\|h^{i+1}\right\|_{L^{\infty}} & \leq \frac{1}{8} \sup _{0 \leq l \leq k}\left\{\left\|h^{i-l}\right\|_{L^{\infty}}\right\}+C\left\{|w r|_{L^{\infty}\left(\gamma_{-}\right)}+\left\|\nu^{-1} w g\right\|_{L^{\infty}}\right\}+C \sup _{0 \leq l \leq k}\left\{\left\|f^{i-l}\right\|_{\nu}\right\} \\
& \leq \frac{1}{8} \sup _{0 \leq l \leq k}\left\{\left\|h^{i-l}\right\|_{L^{\infty}}\right\}+C_{\varepsilon, n}\left\{|w r|_{L^{\infty}\left(\gamma_{-}\right)}+\left\|\nu^{-1} w g\right\|_{L^{\infty}}\right\},
\end{aligned}
$$

where we have used (3.47) in the second inequality. Now we apply Lemma 6.1 to obtain that for $i \geq k+1$,

$$
\begin{aligned}
\left\|h^{i}\right\|_{L^{\infty}} \leq & \frac{1}{8} \max _{0 \leq l \leq k}\left\{\left\|h^{1}\right\|_{L^{\infty}},\left\|h^{2}\right\|_{L^{\infty}}, \cdots,\left\|h^{2 k}\right\|_{L^{\infty}}\right\} \\
& +\frac{8+k}{7} C_{\varepsilon, n}\left\{|w r|_{L^{\infty}\left(\gamma_{-}\right)}+\left\|\nu^{-1} w g\right\|_{L^{\infty}}\right\} \\
\leq & C_{\varepsilon, n, k}\left\{|w r|_{L^{\infty}\left(\gamma_{-}\right)}+\left\|\nu^{-1} w g\right\|_{L^{\infty}}\right\},
\end{aligned}
$$

where we have used (3.45) in the second inequality. Hence it follows from (3.48) and (3.45) that

$$
\left\|h^{i}\right\|_{L^{\infty}} \leq C_{\varepsilon, n, k}\left\{|w r|_{L^{\infty}\left(\gamma_{-}\right)}+\left\|\nu^{-1} w g\right\|_{L^{\infty}}\right\}, \quad \text { for } i \geq 1 .
$$

Taking the difference $h^{i+1}-h^{i}$ and then applying Lemma 3.4 to $h^{i+1}-h^{i}$, we have that for $i \geq k$,

$$
\begin{aligned}
& \left\|h^{i+2}-h^{i+1}\right\|_{L^{\infty}} \\
& \leq \frac{1}{8} \max _{0 \leq l \leq k}\left\{\left\|h^{i+1-l}-h^{i-l}\right\|_{L^{\infty}}\right\}+C \sup _{0 \leq l \leq k}\left\{\left\|f^{i+1-l}-f^{i-l}\right\|_{\nu}\right\} \\
& \leq \frac{1}{8} \max _{0 \leq l \leq k}\left\{\left\|h^{i+1-l}-h^{i-l}\right\|_{L^{\infty}}\right\}+C_{\varepsilon} \cdot\left\{|w r|_{L^{\infty}\left(\gamma_{-}\right)}+\left\|\nu^{-1} w g\right\|_{L^{\infty}}\right\} \cdot \eta_{n}^{i-k} \\
& \leq \frac{1}{8} \max _{0 \leq l \leq k}\left\{\left\|h^{i+1-l}-h^{i-l}\right\|_{L^{\infty}}\right\}+C_{\varepsilon, k}\left\{|w r|_{L^{\infty}\left(\gamma_{-}\right)}+\left\|\nu^{-1} w g\right\|_{L^{\infty}}\right\} \cdot \eta_{n}^{i+k+1},
\end{aligned}
$$


where we have denoted $\eta_{n}:=\left(1-\frac{2}{n}+\frac{3}{2 n^{2}}\right)^{1 / 2}<1$. Here we choose $n$ large enough so that $\frac{1}{8} \eta_{n}^{-k-1} \leq \frac{1}{2}$, then it follows from (3.50) and Lemma 6.1 that

$$
\begin{aligned}
\left\|h^{i+2}-h^{i+1}\right\|_{L^{\infty}} \leq & \left(\frac{1}{8}\right)^{\left[\frac{i}{k+1}\right]} \max _{0 \leq l \leq 2 k}\left\{\left\|h^{1}\right\|_{L^{\infty}},\left\|h^{2}\right\|_{L^{\infty}}, \cdots,\left\|h^{2 k+1}\right\|_{L^{\infty}}\right\} \\
& +C_{\varepsilon, k}\left\{|w r|_{L^{\infty}\left(\gamma_{-}\right)}+\left\|\nu^{-1} w g\right\|_{L^{\infty}}\right\} \cdot \eta_{n}^{i} \\
\leq & C_{\varepsilon, n, k} \cdot\left\{|w r|_{L^{\infty}\left(\gamma_{-}\right)}+\left\|\nu^{-1} w g\right\|_{L^{\infty}}\right\} \cdot\left\{\left(\frac{1}{8}\right)^{\left[\frac{i}{k+1}\right]}+\eta_{n}^{i}\right\},
\end{aligned}
$$

for $i \geq k+1$. Then (3.51) implies immediately that $\left\{h^{i}\right\}_{i=0}^{\infty}$ is a Cauchy sequence in $L^{\infty}$, i.e., there exists a limit function $h \in L^{\infty}$ so that $\left\|h^{i}-h\right\|_{L^{\infty}} \rightarrow 0$ as $i \rightarrow \infty$. Thus we obtained a function $f:=\frac{h}{w}$ solves

$$
\left\{\begin{array}{l}
\mathcal{L}_{0} f=\varepsilon f+v \cdot \nabla_{x} f+\nu(v) f=g \\
\left.f(x, v)\right|_{\gamma_{-}}=\left(1-\frac{1}{n}\right) P_{\gamma} f+r
\end{array}\right.
$$

with $n \geq n_{0}$ large enough. Moreover, from (3.49), there exists a constant $C_{\varepsilon, n, k}$ such that

$$
\|h\|_{L^{\infty}}+|h|_{L^{\infty}(\gamma)} \leq C_{\varepsilon, n, k}\left\{|w r|_{L^{\infty}(\gamma-)}+\left\|\nu^{-1} w g\right\|_{L^{\infty}}\right\} .
$$

Step 2. A priori estimates. For any given $\lambda \in[0,1]$, let $f^{n}$ be the solution of (3.39), i.e.,

$$
\left\{\begin{array}{l}
\mathcal{L}_{\lambda} f^{n}=\varepsilon f^{n}+v \cdot \nabla_{x} f^{n}+\nu(v) f^{n}-\lambda K f^{n}=g, \\
\left.f^{n}(x, v)\right|_{\gamma_{-}}=\left(1-\frac{1}{n}\right) P_{\gamma} f^{n}+r(x, v) .
\end{array}\right.
$$

Moreover we also assume that $\left\|w f^{n}\right\|_{L^{\infty}}+\left|w f^{n}\right|_{L^{\infty}(\gamma)}<\infty$. Firstly, we shall consider a priori $L^{2}$-estimates. Multiplying (3.52) by $f^{n}$, one has that

$$
\begin{aligned}
& \varepsilon\left\|f^{n}\right\|_{L^{2}}^{2}+\frac{1}{2}\left|f^{n}\right|_{L^{2}\left(\gamma_{+}\right)}^{2}-\frac{1}{2}\left|\left(1-\frac{1}{n}\right) P_{\gamma} f^{n}+r\right|_{L^{2}\left(\gamma_{-}\right)}^{2}+\left\|f^{n}\right\|_{\nu}^{2} \\
& \leq \lambda\left\langle K f^{n}, f^{n}\right\rangle+\frac{\varepsilon}{4}\left\|f^{n}\right\|_{L^{2}}+\frac{C}{\varepsilon}\|g\|_{L^{2}}^{2} .
\end{aligned}
$$

We note that $\left\langle L f^{n}, f^{n}\right\rangle \geq 0$, which implies that

$$
\lambda\left\langle K f^{n}, f^{n}\right\rangle \leq \lambda\left\|f^{n}\right\|_{\nu}
$$

On the other hand, a direct computation shows that

$$
\left|\left(1-\frac{1}{n}\right) P_{\gamma} f^{n}+r\right|_{L^{2}\left(\gamma_{-}\right)}^{2} \leq\left(1-\frac{2}{n}+\frac{3}{2 n^{2}}\right)\left|f^{n}\right|_{L^{2}\left(\gamma_{+}\right)}+C_{n}|r|_{L^{2}\left(\gamma_{-}\right)}^{2} .
$$

Substituting (3.54) and (3.55) into (3.53), one has that

$$
\left\|\mathcal{L}_{\lambda}^{-1} g\right\|_{L^{2}}^{2}+\left|\mathcal{L}_{\lambda}^{-1} g\right|_{L^{2}\left(\gamma_{+}\right)}^{2}=\left\|f^{n}\right\|_{L^{2}}^{2}+\left|f^{n}\right|_{L^{2}\left(\gamma_{+}\right)}^{2} \leq C_{\varepsilon, n}\left[|r|_{L^{2}\left(\gamma_{-}\right)}^{2}+\|g\|_{L^{2}}^{2}\right] .
$$

Let $h^{n}:=w f^{n}$. Then, by using (3.12) and (3.56), we obtain

$$
\left\|w \mathcal{L}_{\lambda}^{-1} g\right\|_{L^{\infty}}=\left\|h^{n}\right\|_{L^{\infty}} \leq C_{\varepsilon, n}\left\{|w r|_{L^{\infty}\left(\gamma_{-}\right)}+\left\|\nu^{-1} w g\right\|_{L^{\infty}}\right\} .
$$

On the other hand, Let $\nu^{-1} w g_{1} \in L^{\infty}$ and $\nu^{-1} w g_{2} \in L^{\infty}$. Let $f_{1}^{n}=\mathcal{L}_{\lambda}^{-1} g_{1}$ and $f_{2}^{n}=\mathcal{L}_{\lambda}^{-1} g_{2}$ be the solutions to (3.52) with $g$ replaced by $g_{1}$ and $g_{2}$, respectively. Then we have that

$$
\left\{\begin{array}{l}
\varepsilon\left(f_{2}^{n}-f_{1}^{n}\right)+v \cdot \nabla_{x}\left(f_{2}^{n}-f_{1}^{n}\right)+\nu(v)\left(f_{2}^{n}-f_{1}^{n}\right)-\lambda K\left(f_{2}^{n}-f_{1}^{n}\right)=g_{2}-g_{1}, \\
\left.\left(f_{2}^{n}-f_{1}^{n}\right)(x, v)\right|_{\gamma_{-}}=\left(1-\frac{1}{n}\right) P_{\gamma}\left(f_{2}^{n}-f_{1}^{n}\right) .
\end{array}\right.
$$


By similar arguments as in (3.52)-(3.57), we obtain

$$
\left\|\mathcal{L}_{\lambda}^{-1} g_{2}-\mathcal{L}_{\lambda}^{-1} g_{1}\right\|_{L^{2}}^{2}+\left|\mathcal{L}_{\lambda}^{-1} g_{2}-\mathcal{L}_{\lambda}^{-1} g_{1}\right|_{L^{2}\left(\gamma_{+}\right)}^{2} \leq C_{\varepsilon, n}\left\|g_{2}-g_{1}\right\|_{L^{2}}^{2},
$$

and

$$
\left\|w\left(\mathcal{L}_{\lambda}^{-1} g_{2}-\mathcal{L}_{\lambda}^{-1} g_{1}\right)\right\|_{L^{\infty}} \leq C_{\varepsilon, n}\left\|\nu^{-1} w\left(g_{2}-g_{1}\right)\right\|_{L^{\infty}}
$$

The uniqueness of solution to (3.52) also follows from (3.58). We point out that the constant $C_{\varepsilon, n}$ in (3.56), (3.57), (3.58) and (3.59) does not depend on $\lambda \in[0,1]$. This property is crucial for us to extend $\mathcal{L}_{0}^{-1}$ to $\mathcal{L}_{1}^{-1}$ by a bootstrap argument.

Step 3. In this step, we shall prove the existence of solution $f^{n}$ to (3.39) for sufficiently small $0<\lambda \ll 1$, i.e., to prove the existence of operator $\mathcal{L}_{\lambda}^{-1}$. Firstly, we define the Banach space

$$
\mathbf{X}:=\left\{f=f(x, v): w f \in L^{\infty}\left(\Omega \times \mathbb{R}^{3}\right), w f \in L^{\infty}(\gamma), \text { and }\left.f(x, v)\right|_{\gamma_{-}}=\left(1-\frac{1}{n}\right) P_{\gamma} f+r\right\} .
$$

Now we define

$$
T_{\lambda} f=\mathcal{L}_{0}^{-1}(\lambda K f+g) .
$$

For any $f_{1}, f_{2} \in \mathbf{X}$, by using (3.59), we have that

$$
\begin{aligned}
\left\|w\left(T_{\lambda} f_{1}-T_{\lambda} f_{2}\right)\right\|_{L^{\infty}} & =\left\|w\left\{\mathcal{L}_{0}^{-1}\left(\lambda K f_{1}+g\right)-\mathcal{L}_{0}^{-1}\left(\lambda K f_{2}+g\right)\right\}\right\|_{L^{\infty}} \\
& \leq C_{\varepsilon, n}\left\|\nu^{-1} w\left\{\left(\lambda K f_{1}+g\right)-\left(\lambda K f_{2}+g\right)\right\}\right\|_{L^{\infty}} \\
& \leq \lambda\left\|\nu^{-1} w\left(K f_{1}-K f_{2}\right)\right\|_{L^{\infty}} \\
& \leq \lambda C_{K, \varepsilon, n}\left\|w\left(f_{1}-f_{2}\right)\right\|_{L^{\infty}},
\end{aligned}
$$

where we have used (2.11) and (2.13) with $m=1$ in the last inequality. We take $\lambda_{*}>0$ sufficiently small such that $\lambda_{*} C_{K, \varepsilon, n} \leq 1 / 2$, then $T_{\lambda}: \mathbf{X} \rightarrow \mathbf{X}$ is a contraction mapping for $\lambda \in\left[0, \lambda_{*}\right]$. Thus $T_{\lambda}$ has a fixed point, i.e., $\exists f^{\lambda} \in \mathbf{X}$ such that

$$
f^{\lambda}=T_{\lambda} f^{\lambda}=\mathcal{L}_{0}^{-1}\left(\lambda K f^{\lambda}+g\right)
$$

which yields immediately that

$$
\mathcal{L}_{\lambda} f^{\lambda}=\varepsilon f^{\lambda}+v \cdot \nabla_{x} f^{\lambda}+\nu f^{\lambda}-\lambda K f^{\lambda}=g .
$$

Hence, for any $\lambda \in\left[0, \lambda_{*}\right]$, we have solved (3.39) with $f^{\lambda}=\mathcal{L}_{\lambda}^{-1} g \in \mathbf{X}$. Therefore we have obtained the existence of $\mathcal{L}_{\lambda}^{-1}$ for $\lambda \in\left[0, \lambda_{*}\right]$. Moreover the operator $\mathcal{L}_{\lambda}^{-1}$ has the properties (3.56), (3.57), (3.58) and (3.59).

Next we define

$$
T_{\lambda_{*}+\lambda} f=\mathcal{L}_{\lambda_{*}}^{-1}(\lambda K f+g) .
$$

Noting the estimates for $\mathcal{L}_{\lambda_{*}}^{-1}$ are independent of $\lambda_{*}$. By similar arguments, we can prove $T_{\lambda_{*}+\lambda}$ : $\mathbf{X} \rightarrow \mathbf{X}$ is a contraction mapping for $\lambda \in\left[0, \lambda_{*}\right]$. Then we obtain the exitence of operator $\mathcal{L}_{\lambda_{*}+\lambda}^{-1}$, and (3.56), (3.57), (3.58) and (3.59). Step by step, we can finally obtain the existence of operator $\mathcal{L}_{1}^{-1}$, and $\mathcal{L}_{1}^{-1}$ satisfies the estimates in (3.56), (3.57), (3.58) and (3.59). The continuity is easy to obtain since the convergence of sequence under consideration is always in $L^{\infty}$. Therefore we complete the proof of Lemma 3.5 .

Lemma 3.6. Let $\varepsilon>0$ and $\beta>3$, and assume $\left\|\nu^{-1} w g\right\|_{L^{\infty}}+|w r|_{L^{\infty}\left(\gamma_{-}\right)}<\infty$. Then there exists a unique solution $f^{\varepsilon}$ to solve the approximate linearized steady Boltzmann equation (3.1). Moreover, it satisfies

$$
\left\|w f^{\varepsilon}\right\|_{L^{\infty}}+\left|w f^{\varepsilon}\right|_{L^{\infty}(\gamma)} \leq C_{\varepsilon}\left\{|w r|_{L^{\infty}\left(\gamma_{-}\right)}+\left\|\nu^{-1} w g\right\|_{L^{\infty}}\right\},
$$


where the positive constant $C_{\varepsilon}>0$ depends only on $\varepsilon$. Moreover, if $\Omega$ is a strictly convex domain, $g$ is continuous in $\Omega \times \mathbb{R}^{3}$ and $r$ is continuous in $\gamma_{-}$, then $f^{\varepsilon}$ is continuous away from the grazing set $\gamma_{0}$.

Proof. Let $f^{n}$ be the solution of (3.38) constructed in Lemma 3.5 for $n \geq n_{0}$ with $n_{0}$ large enough. Multiplying (3.38) by $f^{n}$ and using the coercivity estimate (2.7), one obtains that

$$
\varepsilon\left\|f^{n}\right\|_{L^{2}}^{2}+\left|f^{n}\right|_{L^{2}\left(\gamma_{+}\right)}^{2}+2 c_{0}\left\|(I-P) f^{n}\right\|_{\nu}^{2} \leq C_{\varepsilon}\|g\|_{L^{2}}^{2}+\left|\left(1-\frac{1}{n}\right) P_{\gamma} f^{n}+r\right|_{L^{2}\left(\gamma_{-}\right)}^{2} .
$$

Here the projection $P$ is defined by (2.6). A direct calculation shows that

$$
\begin{aligned}
\left|\left(1-\frac{1}{n}\right) P_{\gamma} f^{n}+r\right|_{L^{2}\left(\gamma_{-}\right)}^{2} & \leq\left|P_{\gamma} f^{n}\right|_{L^{2}\left(\gamma_{-}\right)}^{2}+2\left|P_{\gamma} f^{n}\right|_{L^{2}\left(\gamma_{-}\right)} \cdot|r|_{L^{2}\left(\gamma_{-}\right)}+|r|_{L^{2}\left(\gamma_{-}\right)}^{2} \\
& \leq\left|P_{\gamma} f^{n}\right|_{L^{2}\left(\gamma_{+}\right)}^{2}+\eta\left|P_{\gamma} f^{n}\right|_{L^{2}\left(\gamma_{+}\right)}^{2}+C_{\eta}|r|_{L^{2}\left(\gamma_{-}\right)}^{2}
\end{aligned}
$$

which together with (3.61) yield that

$$
\varepsilon\left\|f^{n}\right\|_{L^{2}}^{2}+\left|\left(I-P_{\gamma}\right) f^{n}\right|_{\gamma_{+}}^{2}+2 c_{0}\left\|(I-P) f^{n}\right\|_{\nu}^{2} \leq \eta\left|P_{\gamma} f^{n}\right|_{L^{2}\left(\gamma_{+}\right)}^{2}+C_{\varepsilon, \eta}\left(\|g\|_{L^{2}}^{2}+|r|_{L^{2}\left(\gamma_{-}\right)}^{2}\right) \text {. }
$$

where $\eta>0$ is a small constant to be chosen later.

We still need to bound the first term on the right-hand side of (3.62). Firstly a direct calculation shows that

$$
\frac{1}{2}\left|P_{\gamma} f^{n}\right|_{L^{2}\left(\gamma_{+}\right)}^{2} \leq\left|P_{\gamma} f^{n} I_{\gamma_{+} \backslash \gamma_{+}^{\varepsilon^{e^{\prime}}}}\right|_{L^{2}\left(\gamma_{+}\right)}^{2},
$$

provided that $0<\varepsilon^{\prime} \ll 1$. We note that

$$
f^{n}(x, v)=\left(I-P_{\gamma}\right) f^{n}(x, v)+P_{\gamma} f^{n}(x, v), \quad \forall(x, v) \in \gamma_{+},
$$

which yields that

$$
\left|P_{\gamma} f^{n} I_{\gamma_{+} \backslash \gamma_{+}^{\varepsilon^{\varepsilon}}}\right|_{L^{2}(\gamma)}^{2}=2\left|f^{n} I_{\gamma_{+} \backslash \gamma_{+}^{\varepsilon^{\prime}}}\right|_{L^{2}(\gamma)}^{2}+2\left|\left(I-P_{\gamma}\right) f^{n} I_{\gamma_{+} \backslash \gamma_{+}^{\varepsilon^{\prime}}}\right|_{L^{2}(\gamma)}^{2} .
$$

On the other hand, it follows from (3.38) that

$$
\frac{1}{2} v \cdot \nabla_{x}\left(\left|f^{n}\right|^{2}\right)=-\varepsilon\left|f^{n}\right|^{2}-f^{n} L f^{n}+g f^{n},
$$

which yields that

$$
\left\|v \cdot \nabla_{x}\left(\left|f^{n}\right|^{2}\right)\right\|_{L^{1}} \leq C\left\{\left\|f^{n}\right\|_{L^{2}}^{2}+\left\|(I-P) f^{n}\right\|_{\nu}^{2}+\|g\|_{L^{2}}^{2}\right\} .
$$

It follows from (3.65) and (3.62) that

$$
\begin{aligned}
\left|f^{n} I_{\gamma_{+} \backslash \gamma_{+}^{\varepsilon^{\prime}}}\right|_{L^{2}(\gamma)}^{2} & =\left|\left(f^{n}\right)^{2} I_{\gamma_{+} \backslash \gamma_{+}^{\varepsilon^{\prime}}}\right|_{L^{1}(\gamma)} \\
& \leq C_{\varepsilon^{\prime}}\left\{\left\|f^{n}\right\|_{L^{2}}^{2}+\left\|v \cdot \nabla_{x}\left(\left|f^{n}\right|^{2}\right)\right\|_{L^{1}}\right\} \\
& \leq C_{\varepsilon^{\prime}}\left\{\left\|f^{n}\right\|_{L^{2}}^{2}+\left\|(I-P) f^{n}\right\|_{\nu}^{2}+\|g\|_{L^{2}}^{2}\right\} \\
& \leq C_{\varepsilon^{\prime}, \varepsilon} \eta\left|P_{\gamma} f^{n}\right|_{L^{2}\left(\gamma_{+}\right)}^{2}+C_{\varepsilon^{\prime}, \varepsilon, \eta}\left\{\|g\|_{L^{2}}^{2}+|r|_{L^{2}\left(\gamma_{-}\right)}^{2}\right\},
\end{aligned}
$$

where in the second line we have used (4.8) which will be given in Lemma 4.1 later on. The above estimate together with (3.63) and (3.64) yield that

$$
\left|P_{\gamma} f^{n}\right|_{L^{2}\left(\gamma_{+}\right)}^{2} \leq C_{\varepsilon^{\prime}, \varepsilon} \eta\left|P_{\gamma} f^{n}\right|_{L^{2}\left(\gamma_{+}\right)}^{2}+C_{\varepsilon^{\prime}, \varepsilon, \eta}\left\{\|g\|_{L^{2}}^{2}+|r|_{L^{2}\left(\gamma_{-}\right)}^{2}\right\} .
$$

Taking $\eta$ small so that $C_{\varepsilon^{\prime}, v} \eta \leq \frac{1}{2}$, one obtains that

$$
\left|P_{\gamma} f^{n}\right|_{L^{2}\left(\gamma_{+}\right)}^{2} \leq C_{\varepsilon^{\prime}, \varepsilon}\left\{\|g\|_{L^{2}}^{2}+|r|_{L^{2}\left(\gamma_{-}\right)}^{2}\right\} .
$$


Combining (3.66) and (3.62), one has

$$
\left\|f^{n}\right\|_{L^{2}}^{2}+\left|f^{n}\right|_{L^{2}\left(\gamma_{+}\right)}^{2}+2 c_{0}\left\|(I-P) f^{n}\right\|_{\nu}^{2} \leq C_{\varepsilon^{\prime}, \varepsilon}\left(\|g\|_{L^{2}}^{2}+|r|_{L^{2}\left(\gamma_{-}\right)}^{2}\right) .
$$

We apply (3.12) and use (3.67) to obtain

$$
\begin{aligned}
\left\|w f^{n}\right\|_{L^{\infty}}+\left|w f^{n}\right|_{L^{\infty}(\gamma)} & \leq C\left\{\left\|\nu^{-1} w g\right\|_{L^{\infty}}+|w r|_{L^{\infty}\left(\gamma_{-}\right)}+\left\|f^{n}\right\|_{L^{2}}\right\} \\
& \leq C_{\varepsilon^{\prime}, \varepsilon}\left\{\left\|\nu^{-1} w g\right\|_{L^{\infty}}+|w r|_{L^{\infty}\left(\gamma_{-}\right)}\right\}
\end{aligned}
$$

Taking the difference $f^{n_{1}}-f^{n_{2}}$ with $n_{1}, n_{2} \geq n_{0}$, we know that

$$
\left\{\begin{array}{l}
\varepsilon\left(f^{n_{1}}-f^{n_{2}}\right)+v \cdot \nabla_{x}\left(f^{n_{1}}-f^{n_{2}}\right)+L\left(f^{n_{1}}-f^{n_{2}}\right)=0, \\
\left.\left(f^{n_{1}}-f^{n_{2}}\right)(x, v)\right|_{\gamma_{-}}=\left(1-\frac{1}{n_{1}}\right) P_{\gamma}\left(f^{n_{1}}-f^{n_{2}}\right)+\left(\frac{1}{n_{2}}-\frac{1}{n_{1}}\right) P_{\gamma} f^{n_{2}} .
\end{array}\right.
$$

Multiplying (3.68) by $f^{n_{1}}-f^{n_{2}}$, and integrating it over $\Omega \times \mathbb{R}^{3}$, by similar arguments as in (3.61)(3.67), we can obtain

$$
\begin{aligned}
& \left\|\left(f^{n_{1}}-f^{n_{2}}\right)\right\|_{L^{2}}^{2}+\left|\left(f^{n_{1}}-f^{n_{2}}\right)\right|_{L^{2}\left(\gamma_{+}\right)}^{2}+2 c_{0}\left\|(I-P)\left(f^{n_{1}}-f^{n_{2}}\right)\right\|_{\nu}^{2} \\
& \leq C_{\varepsilon^{\prime}, \varepsilon}\left|\left(\frac{1}{n_{2}}-\frac{1}{n_{1}}\right) P_{\gamma} f^{n_{2}}\right|_{L^{2}\left(\gamma_{-}\right)}^{2} \leq C_{\varepsilon^{\prime}, \varepsilon} \cdot\left\{\left(\frac{1}{n_{1}}\right)^{2}+\left(\frac{1}{n_{2}}\right)^{2}\right\} \cdot\left|f^{n_{2}}\right|_{L^{2}\left(\gamma_{+}\right)}^{2} \\
& \leq C_{\varepsilon^{\prime}, \varepsilon} \cdot\left(\|g\|_{L^{2}}^{2}+|r|_{L^{2}\left(\gamma_{-}\right)}^{2}\right) \cdot\left\{\left(\frac{1}{n_{1}}\right)^{2}+\left(\frac{1}{n_{2}}\right)^{2}\right\} \rightarrow 0,
\end{aligned}
$$

as $n_{1}, n_{2} \rightarrow \infty$, where we have used the uniform estimate (3.67) in the last inequality. Applying (3.12) to $f^{n_{1}}-f^{n_{2}}$ and using (3.69), then one has

$$
\begin{aligned}
\left\|w\left(f^{n_{1}}-f^{n_{2}}\right)\right\|_{L^{\infty}}+\left|w\left(f^{n_{1}}-f^{n_{2}}\right)\right|_{L^{\infty}(\gamma)} & \leq C\left|w\left(\frac{1}{n_{2}}-\frac{1}{n_{1}}\right) P_{\gamma} f^{n_{2}}\right|_{L^{\infty}\left(\gamma_{-}\right)}+C\left\|f^{n_{1}}-f^{n_{2}}\right\|_{L^{2}} \\
& \leq C_{\varepsilon^{\prime}, \varepsilon} \cdot\left(\|w g\|_{L^{\infty}}+|w r|_{L^{\infty}\left(\gamma_{-}\right)}\right) \cdot\left\{\frac{1}{n_{1}}+\frac{1}{n_{2}}\right\} \rightarrow 0,
\end{aligned}
$$

as $n_{1}, n_{2} \rightarrow \infty$, which yields that $w f^{n}$ is a Cauchy sequence in $L^{\infty}$. We denote $f^{\varepsilon}=\lim _{n \rightarrow \infty} f^{n}$, then it is direct to check that $f^{\varepsilon}$ is a solution to (3.1), and (3.60) holds. The continuity of $f^{\varepsilon}$ is easy to obtain since the convergence of sequences is always in $L^{\infty}$ and $f^{n}$ is continuous away from the grazing set. Therefore we have completed the proof of Lemma 3.6.

Now we assume

$$
\iint_{\Omega \times \mathbb{R}^{3}} g(x, v) \mu^{\frac{1}{2}}(v) \mathrm{d} v \mathrm{~d} x=\int_{\gamma_{-}} r(x, v) \mu^{\frac{1}{2}}(v) \mathrm{d} \gamma=0 .
$$

Lemma 3.7. Let $f$ be a solution

$$
v \cdot \nabla_{x} f+L f=g, \quad f_{\gamma_{-}}=P_{\gamma} f+r
$$

in the weak sense of

$$
\int_{\gamma} \psi f\{v \cdot n(x)\} \mathrm{d} v \mathrm{~d} S_{x}-\int_{\Omega \times \mathbb{R}^{3}} v \cdot \nabla_{x} \psi f \mathrm{~d} v \mathrm{~d} x=-\int_{\Omega \times \mathbb{R}^{3}} \psi L f \mathrm{~d} v \mathrm{~d} x+\int_{\Omega \times \mathbb{R}^{3}} \psi g \mathrm{~d} v \mathrm{~d} x,
$$

where $\psi \in H^{1}\left(\Omega \times \mathbb{R}^{3}\right)$. Assume $\int_{\Omega \times \mathbb{R}^{3}} f \sqrt{\mu} \mathrm{d} v \mathrm{~d} x=0$ and (3.70), then it holds that

$$
\|P f\|_{L^{2}}^{2} \leq C\left\{\|(I-P) f\|_{\nu}^{2}+\|g\|_{L^{2}}+\left|\left(I-P_{\gamma}\right) f\right|_{L^{2}\left(\gamma_{+}\right)}^{2}+|r|_{L^{2}\left(\gamma_{-}\right)}^{2}\right\} .
$$

Proof. The proof is almost the same to Lemma 3.3 in [21, the details are omitted here for simplicity of presentation. 
Lemma 3.8. Assume (3.70). Let $\beta>3+|\kappa|$, and assume $\left\|\nu^{-1} w g\right\|_{L^{\infty}}+|w r|_{L^{\infty}\left(\gamma_{-}\right)}<\infty$. Then there exists a unique solution $f=f(x, v)$ to the linearized steady Boltzmann equation

$$
v \cdot \nabla_{x} f+L f=g,\left.\quad f(x, v)\right|_{\gamma_{-}}=P_{\gamma} f+r,
$$

such that $\int_{\Omega \times \mathbb{R}^{3}} f \sqrt{\mu} d v d x=0$ and

$$
\|w f\|_{L^{\infty}}+|w f|_{L^{\infty}(\gamma)} \leq C\left\{|w r|_{L^{\infty}\left(\gamma_{-}\right)}+\left\|\nu^{-1} w g\right\|_{L^{\infty}}\right\} .
$$

Moreover, if $\Omega$ is a strictly convex domain, $g$ is continuous in $\Omega \times \mathbb{R}$ and $r$ is continuous in $\gamma_{-}$, then $f$ is continuous away from the grazing set $\gamma_{0}$.

Proof. Let $f^{\varepsilon}$ be the solution of (3.1) constructed in Lemma 3.6 for $\varepsilon>0$. Multiplying the first equation of (3.1) by $\sqrt{\mu}$, taking integration over $\Omega \times \mathbb{R}^{3}$, and noting (3.70), it is straightforward to see that

$$
\int_{\Omega \times \mathbb{R}^{3}} f^{\varepsilon}(x, v) \mu^{\frac{1}{2}}(v) \mathrm{d} v \mathrm{~d} x=0,
$$

for any $\varepsilon>0$. Multiplying the first equation of (3.1) by $f^{\varepsilon}$ and integrating the resultant equation over $\Omega \times \mathbb{R}^{3}$, it follows from Cauchy inequality that

$$
\begin{aligned}
& \varepsilon\left\|f^{\varepsilon}\right\|_{L^{2}}^{2}+\frac{1}{2}\left|\left(I-P_{\gamma}\right) f^{\varepsilon}\right|_{L^{2}\left(\gamma_{+}\right)}^{2}+c_{0}\left\|(I-P) f^{\varepsilon}\right\|_{\nu}^{2} \\
& \leq \eta\left[\left\|f^{\varepsilon}\right\|_{\nu}^{2}+\left|P_{\gamma} f^{\varepsilon}\right|_{L^{2}\left(\gamma_{+}\right)}^{2}\right]+C_{\eta}\left[\left\|\nu^{-\frac{1}{2}} g\right\|_{L^{2}}^{2}+|r|_{L^{2}\left(\gamma_{-}\right)}^{2}\right] .
\end{aligned}
$$

Applying Lemma 3.7 to $f^{\varepsilon}$, we obtain

$$
\left\|P f^{\varepsilon}\right\|_{L^{2}}^{2} \leq C\left\{\left\|(I-P) f^{\varepsilon}\right\|_{\nu}^{2}+\varepsilon\left\|f^{\varepsilon}\right\|_{L^{2}}^{2}+\left|\left(I-P_{\gamma}\right) f^{\varepsilon}\right|_{L^{2}\left(\gamma_{+}\right)}^{2}+\|g\|_{L^{2}}^{2}+|r|_{L^{2}\left(\gamma_{-}\right)}^{2}\right\},
$$

which together with (3.73) imply that

$$
\begin{aligned}
\varepsilon\left\|f^{\varepsilon}\right\|_{L^{2}}^{2}+\left\|f^{\varepsilon}\right\|_{\nu}^{2}+\left|\left(I-P_{\gamma}\right) f^{\varepsilon}\right|_{L^{2}\left(\gamma_{+}\right)}^{2} & \\
& \leq C \eta\left[\left\|f^{\varepsilon}\right\|_{\nu}^{2}+\left|P_{\gamma} f^{\varepsilon}\right|_{L^{2}\left(\gamma_{+}\right)}^{2}\right]+C_{\eta}\left[\left\|\nu^{-\frac{1}{2}} g\right\|_{L^{2}}^{2}+|r|_{L^{2}\left(\gamma_{-}\right)}^{2}\right],
\end{aligned}
$$

where $\eta>0$ is a small positive constant to be determined later.

To control the term $\left|P_{\gamma} f^{\varepsilon}\right|_{L^{2}\left(\gamma_{+}\right)}^{2}$ on the right-hand side of (3.74), we should be careful since we do not have the uniform bound on $\left\|f^{\varepsilon}\right\|_{L^{2}}$. Denote

$$
z_{\gamma}^{\varepsilon}(x):=\int_{v^{\prime} \cdot n(x)>0} f^{\varepsilon}\left(x, v^{\prime}\right) \sqrt{\mu\left(v^{\prime}\right)}\left\{v^{\prime} \cdot n(x)\right\} \mathrm{d} v^{\prime},
$$

then one has $P_{\gamma} f^{\varepsilon}=z_{\gamma}^{\varepsilon}(x) \mu^{\frac{1}{2}}(v)$. A direct calculation shows that

$$
\int_{v \cdot n(x) \geq \varepsilon^{\prime}, \varepsilon^{\prime} \leq|v| \leq \frac{1}{\varepsilon^{\prime}}} \nu(v) \mu(v)|v \cdot n(x)| \mathrm{d} v \geq c_{1}>0,
$$

provided that $0<\varepsilon^{\prime} \ll 1$, where $c_{1}>0$ is a positive constant independent of $\varepsilon^{\prime}$. By using (3.75), we have that

$$
\begin{aligned}
\left|\sqrt{\nu} P_{\gamma} f^{\varepsilon} I_{\gamma_{+} \backslash \gamma_{+}^{\varepsilon^{\prime}}}\right|_{L^{2}}^{2} & =\int_{\partial \Omega}\left|z_{\gamma}^{\varepsilon}(x)\right|^{2} \mathrm{~d} S_{x} \cdot \int_{v \cdot n(x) \geq \varepsilon^{\prime}, \varepsilon^{\prime} \leq|v| \leq \frac{1}{\varepsilon^{\prime}}} \nu(v) \mu(v)|v \cdot n(x)| \mathrm{d} v \\
& \geq c_{1}\left|P_{\gamma} f^{\varepsilon}\right|_{L^{2}\left(\gamma_{+}\right)}^{2}
\end{aligned}
$$


which yields immediately that

$$
\begin{aligned}
\left|P_{\gamma} f^{\varepsilon}\right|_{L^{2}\left(\gamma_{+}\right)}^{2} \leq & C\left|\sqrt{\nu} P_{\gamma} f^{\varepsilon} I_{\gamma_{+} \backslash \gamma_{+}^{\varepsilon^{\prime}}}\right|_{L^{2}}^{2} \\
\leq & C\left\{\left|\sqrt{\nu} f^{\varepsilon} I_{\gamma_{+} \backslash \gamma_{+}^{\varepsilon^{\prime}}}\right|_{L^{2}}^{2}+\left|\sqrt{\nu}\left(I-P_{\gamma}\right) f^{\varepsilon} I_{\gamma_{+} \backslash \gamma_{+}^{\varepsilon^{\prime}}}\right|_{L^{2}}^{2}\right\} \\
\leq & C\left|\sqrt{\nu} f^{\varepsilon} I_{\gamma_{+} \backslash \gamma_{+}^{\varepsilon^{\prime}}}\right|_{L^{2}}^{2}+C \eta\left[\left\|f^{\varepsilon}\right\|_{\nu}^{2}+\left|P_{\gamma} f^{\varepsilon}\right|_{L^{2}\left(\gamma_{+}\right)}^{2}\right] \\
& +C_{\eta}\left[\left\|\nu^{-\frac{1}{2}} g\right\|_{L^{2}}^{2}+|r|_{L^{2}\left(\gamma_{-}\right)}^{2}\right] .
\end{aligned}
$$

It follows from (3.1) that

$$
\frac{1}{2} v \cdot \nabla_{x}\left(\nu\left|f^{\varepsilon}\right|^{2}\right)=-\varepsilon \nu\left|f^{\varepsilon}\right|^{2}-\nu f^{\varepsilon} L f^{\varepsilon}+\nu f^{\varepsilon} g
$$

which implies that

$$
\left\|v \cdot \nabla_{x}\left(\nu\left|f^{\varepsilon}\right|^{2}\right)\right\|_{L^{1}} \leq C\left\{\left\|f^{\varepsilon}\right\|_{\nu}^{2}+\|g\|_{\nu}^{2}\right\} .
$$

It follows from (3.77) and (4.8) that

$$
\begin{aligned}
\frac{1}{2}\left|\sqrt{\nu} f^{\varepsilon} I_{\gamma_{+} \backslash \gamma_{+}^{\varepsilon^{\prime}}}\right|_{L^{2}\left(\gamma_{+}\right)}^{2} & =\frac{1}{2}\left|\nu\left(f^{\varepsilon}\right)^{2} I_{\gamma_{+} \backslash \gamma_{+}^{\varepsilon^{\prime}}}\right|_{L^{1}\left(\gamma_{+}\right)} \leq C\left\{\left\|\nu\left(f^{\varepsilon}\right)^{2}\right\|_{L^{1}}+\left\|v \cdot \nabla_{x}\left(\nu\left|f^{\varepsilon}\right|^{2}\right)\right\|_{L^{1}}\right\} \\
& \leq C\left\{\left\|f^{\varepsilon}\right\|_{\nu}^{2}+\|g\|_{\nu}^{2}\right\},
\end{aligned}
$$

which together with (3.76) and by taking $\eta>0$ suitably small, yield that

$$
\left|P_{\gamma} f^{\varepsilon}\right|_{L^{2}\left(\gamma_{+}\right)}^{2} \leq C\left\|f^{\varepsilon}\right\|_{\nu}^{2}+C\left[\left\|\nu^{-\frac{1}{2}} g\right\|_{L^{2}}^{2}+|r|_{L^{2}\left(\gamma_{-}\right)}^{2}\right] .
$$

Combining (3.78) and (3.74), then taking $\eta>0$ small, one has that

$$
\left\|f^{\varepsilon}\right\|_{\nu}^{2}+\left|f^{\varepsilon}\right|_{L^{2}\left(\gamma_{+}\right)}^{2} \leq C\left[\left\|\nu^{-\frac{1}{2}} g\right\|_{L^{2}}^{2}+|r|_{L^{2}\left(\gamma_{-}\right)}^{2}\right] .
$$

Applying (3.12) to $f^{\varepsilon}$ and using (3.79), then we obtain

$$
\left\|w f^{\varepsilon}\right\|_{L^{\infty}}+\left|w f^{\varepsilon}\right|_{L^{\infty}(\gamma)} \leq C\left[\left\|\nu^{-\frac{1}{2}} w g\right\|_{L^{\infty}}+|w r|_{L^{\infty}\left(\gamma_{-}\right)}\right],
$$

Next we consider the convergence of $f^{\varepsilon}$ as $\varepsilon \rightarrow 0+$. For any $\varepsilon_{1}, \varepsilon_{2}>0$, we consider the difference $f^{\varepsilon_{2}}-f^{\varepsilon_{1}}$ satisfying

$$
\left\{\begin{array}{l}
v \cdot \nabla_{x}\left(f^{\varepsilon_{2}}-f^{\varepsilon_{1}}\right)+L\left(f^{\varepsilon_{2}}-f^{\varepsilon_{1}}\right)=-\varepsilon_{2} f^{\varepsilon_{2}}+\varepsilon_{1} f^{\varepsilon_{1}}, \\
\left.\left(f^{\varepsilon_{2}}-f^{\varepsilon_{1}}\right)\right|_{\gamma_{-}}=P_{\gamma}\left(f^{\varepsilon_{2}}-f^{\varepsilon_{1}}\right) .
\end{array}\right.
$$

Multiplying (3.81) by $f^{\varepsilon_{2}}-f^{\varepsilon_{1}}$, integrating the resultant equation and by similar arguments as in (3.73)-(3.79), one gets

$$
\begin{aligned}
& \left\|f^{\varepsilon_{2}}-f^{\varepsilon_{1}}\right\|_{\nu}^{2}+\left|f^{\varepsilon_{2}}-f^{\varepsilon_{1}}\right|_{L^{2}\left(\gamma_{+}\right)}^{2} \\
& \leq C\left\|\nu^{-\frac{1}{2}}\left(\varepsilon_{2} f^{\varepsilon_{2}}-\varepsilon_{1} f^{\varepsilon_{1}}\right)\right\|_{L^{2}}^{2} \leq C\left(\varepsilon_{1}^{2}+\varepsilon_{2}^{2}\right)\left[\left\|w f^{\varepsilon_{1}}\right\|_{L^{\infty}}^{2}+\left\|w f^{\varepsilon_{2}}\right\|_{L^{\infty}}^{2}\right] \\
& \leq C\left(\varepsilon_{1}^{2}+\varepsilon_{2}^{2}\right) \cdot\left[\left\|\nu^{-1} w g\right\|_{L^{\infty}}+|w r|_{L^{\infty}\left(\gamma_{-}\right)}\right]^{2},
\end{aligned}
$$

as $\varepsilon_{1}, \varepsilon_{2} \rightarrow 0+$, where we have used (3.80) in the last inequality. Finally, applying (3.12) to $f^{\varepsilon_{2}}-f^{\varepsilon_{1}}$, then we obtain

$$
\begin{aligned}
\left\|\nu w\left(f^{\varepsilon_{2}}-f^{\varepsilon_{1}}\right)\right\|_{L^{\infty}}+\left|\nu w\left(f^{\varepsilon_{2}}-f^{\varepsilon_{1}}\right)\right|_{L^{\infty}(\gamma)} & \leq C\left\{\left\|w\left(\varepsilon_{2} f^{\varepsilon_{2}}-\varepsilon_{1} f^{\varepsilon_{1}}\right)\right\|_{L^{\infty}}+\left\|f^{\varepsilon_{2}}-f^{\varepsilon_{1}}\right\|_{\nu}\right\} \\
& \leq C\left(\varepsilon_{1}+\varepsilon_{2}\right) \cdot\left[\left\|\nu^{-1} w g\right\|_{L^{\infty}}+|w r|_{L^{\infty}\left(\gamma_{-}\right)}\right]
\end{aligned}
$$

as $\varepsilon_{1}, \varepsilon_{2} \rightarrow 0+$, where we have used (3.82) and (3.80) above. Here we have to demand $\beta>3+|\kappa|$ so that we can apply (3.12). And we point out here that we can only obtain the convergence in a weak norm $L_{\nu w}^{\infty}$ but not $L_{w}^{\infty}$, the main reason is that for soft potentials, in order to get the uniform $L_{w}^{\infty}$ estimate, one has to demand $g$ to has the more velocity weight. With (3.83), we know that there exists a function $f$ so that $\left\|\nu w\left(f^{\varepsilon}-f\right)\right\|_{L^{\infty}} \rightarrow 0$ as $\varepsilon \rightarrow 0+$. And it is direct to see that 
$f$ solves (3.71). Also, (3.72) follows immediately from (3.80). If $\Omega$ is convex, the continuity of $f$ directly follows from the $L^{\infty}$-convergence. Therefore the proof of Lemma 3.8 is complete.

3.3. Proof of Theorem 1.1. We consider the following iterative sequence

$$
\left\{\begin{array}{l}
v \cdot \nabla_{x} f^{j+1}+L f^{j+1}=\Gamma\left(f^{j}, f^{j}\right), \\
\left.f^{j+1}\right|_{\gamma_{-}}=P_{\gamma} f^{j+1}+\frac{\mu_{\theta}-\mu}{\sqrt{\mu}}+\frac{\mu_{\theta}-\mu}{\sqrt{\mu}} \int_{v^{\prime} \cdot n(x)>0} f^{j}\left(x, v^{\prime}\right) \sqrt{\mu\left(v^{\prime}\right)}\left\{v^{\prime} \cdot n(x)\right\} \mathrm{d} v^{\prime},
\end{array}\right.
$$

for $j=0,1,2 \cdots$ with $f^{0} \equiv 0$. A direct computation shows that

$$
\int_{\Omega \times \mathbb{R}^{3}} \Gamma\left(f^{j}, f^{j}\right) \mu^{\frac{1}{2}}(v) \mathrm{d} v \mathrm{~d} x=0, \quad \int_{v \cdot n(x)<0}\left[\mu_{\theta}(v)-\mu(v)\right]\{v \cdot n(x)\} \mathrm{d} v=0,
$$

which yield that

$$
\int_{\gamma_{-}}\left\{\frac{\mu_{\theta}-\mu}{\sqrt{\mu}}+\frac{\mu_{\theta}-\mu}{\sqrt{\mu}} \int_{v^{\prime} \cdot n(x)>0} f^{j}\left(x, v^{\prime}\right) \sqrt{\mu\left(v^{\prime}\right)}\left\{v^{\prime} \cdot n(x)\right\} \mathrm{d} v^{\prime}\right\} \mu^{\frac{1}{2}}(v) \mathrm{d} \gamma \equiv 0 .
$$

We note that

$$
\left\|\nu^{-1} w \Gamma\left(f^{j}, f^{j}\right)\right\|_{L^{\infty}} \leq C\left\|w f^{j}\right\|_{L^{\infty}}^{2}
$$

and

$$
\begin{aligned}
\left|w\left\{\frac{\mu_{\theta}-\mu}{\sqrt{\mu}}+\frac{\mu_{\theta}-\mu}{\sqrt{\mu}} \int_{v^{\prime} \cdot n(x)>0} f^{j}\left(x, v^{\prime}\right) \sqrt{\mu\left(v^{\prime}\right)}\left\{v^{\prime} \cdot n(x)\right\} \mathrm{d} v^{\prime}\right\}\right|_{L^{\infty}\left(\gamma_{-}\right)} & \\
& \leq C \delta+C \delta\left|f^{j}\right|_{L^{\infty}\left(\gamma_{+}\right)} .
\end{aligned}
$$

Noting (3.85)-(3.87), and using Lemma 3.8, we can solve (3.84) inductively for $j=0,1,2, \cdots$. Moreover, it follows from (3.72), (3.86) and (3.87) that

$$
\left\|w f^{j+1}\right\|_{L^{\infty}}+\left|w f^{j+1}\right|_{L^{\infty}(\gamma)} \leq C_{1} \delta+C_{1} \delta\left|f^{j}\right|_{L^{\infty}\left(\gamma_{+}\right)}+C_{1}\left\|w f^{j}\right\|_{L^{\infty}}^{2} .
$$

By induction, we shall prove that

$$
\left\|w f^{j}\right\|_{L^{\infty}}+\left|w f^{j}\right|_{L^{\infty}(\gamma)} \leq 2 C_{1} \delta, \quad \text { for } j=1,2, \cdots .
$$

Indeed, for $j=0$, it follows from $f^{0} \equiv 0$ and (3.88) that

$$
\left\|w f^{1}\right\|_{L^{\infty}}+\left|w f^{1}\right|_{L^{\infty}(\gamma)} \leq C_{1} \delta .
$$

Now we assume that (3.89) holds for $j=1,2 \cdots, l$, then we consider the case for $j=l+1$. Indeed it follows from (3.88) that

$$
\begin{aligned}
\left\|w f^{l+1}\right\|_{L^{\infty}}+\left|w f^{l+1}\right|_{L^{\infty}(\gamma)} & \leq C_{1} \delta+C_{1} \delta\left|f^{l}\right|_{L^{\infty}(\gamma)}+C_{1}\left\|w f^{l}\right\|_{L^{\infty}}^{2} \\
& \leq C_{1} \delta\left(1+2 C_{1} \delta+4 C_{1}^{2} \delta\right) \leq \frac{3}{2} C_{1} \delta
\end{aligned}
$$

where we have used (3.89) with $j=l$, and chosen $\delta>0$ small enough such that $2 C_{1} \delta+4 C_{1}^{2} \delta \leq 1 / 2$. Therefore we have proved (3.89) by induction.

Finally we consider the convergence of sequence $f^{j}$. For the difference $f^{j+1}-f^{j}$, we have

$$
\left\{\begin{aligned}
v \cdot \nabla_{x}\left(f^{j+1}-f^{j}\right) & +L\left(f^{j+1}-f^{j}\right)=\Gamma\left(f^{j}-f^{j-1}, f^{j}\right)+\Gamma\left(f^{j-1}, f^{j}-f^{j-1}\right), \\
\left.\left(f^{j+1}-f^{j}\right)\right|_{\gamma_{-}}= & P_{\gamma}\left(f^{j+1}-f^{j}\right) \\
& +\frac{\mu_{\theta}-\mu}{\sqrt{\mu}} \int_{v^{\prime} \cdot n(x)>0}\left[f^{j}-f^{j-1}\right]\left(x, v^{\prime}\right) \sqrt{\mu\left(v^{\prime}\right)}\left\{v^{\prime} \cdot n(x)\right\} \mathrm{d} v^{\prime} .
\end{aligned}\right.
$$


Applying (3.72) to (3.90), we have that

$$
\begin{aligned}
&\left\|w\left\{f^{j+1}-f^{j}\right\}\right\|_{L^{\infty}}+\left|w\left\{f^{j+1}-f^{j}\right\}\right|_{L^{\infty}(\gamma)} \\
& \leq C\left\{\left\|\nu^{-1} w \Gamma\left(f^{j}-f^{j-1}, f^{j}\right)\right\|_{L^{\infty}}+\left\|\nu^{-1} w \Gamma\left(f^{j-1}, f^{j}-f^{j-1}\right)\right\|_{L^{\infty}}\right\} \\
& \quad+C\left|w\left\{\frac{\mu_{\theta}-\mu}{\sqrt{\mu}} \int_{v^{\prime} \cdot n(x)>0}\left[f^{j}-f^{j-1}\right]\left(x, v^{\prime}\right) \sqrt{\mu\left(v^{\prime}\right)}\left\{v^{\prime} \cdot n(x)\right\} \mathrm{d} v^{\prime}\right\}\right|_{L^{\infty}\left(\gamma_{-}\right)} \\
& \leq C\left[\delta+\left\|w f^{j}\right\|_{L^{\infty}}+\left\|w f^{j-1}\right\|_{L^{\infty}}\right] \cdot\left\{\left\|w\left(f^{j}-f^{j-1}\right)\right\|_{L^{\infty}}+\left|w\left(f^{j}-f^{j-1}\right)\right|_{L^{\infty}\left(\gamma_{+}\right)}\right\} \\
& \leq C \delta\left\{\left\|w\left(f^{j}-f^{j-1}\right)\right\|_{L^{\infty}}+\left|w\left(f^{j}-f^{j-1}\right)\right|_{L^{\infty}\left(\gamma_{+}\right)}\right\} \\
& \leq \frac{1}{2}\left\{\left\|w\left(f^{j}-f^{j-1}\right)\right\|_{L^{\infty}}+\left|w\left(f^{j}-f^{j-1}\right)\right|_{L^{\infty}\left(\gamma_{+}\right)}\right\},
\end{aligned}
$$

where we have used (3.89) and taken $\delta>0$ small such that $C \delta \leq 1 / 2$. Hence $f^{j}$ is a Cauchy sequence in $L^{\infty}$, then we obtain the solution by taking the $\operatorname{limit} f_{*}=\lim _{j \rightarrow \infty} f^{j}$. The uniqueness can also be obtained by using the inequality as (3.91).

If $\Omega$ is convex, the continuity of $f_{*}$ is a direct consequence of $L^{\infty}$-convergence. The positivity of $F_{*}:=\mu+\sqrt{\mu} f_{*}$ will be proved in Section 4. Therefore we complete the proof of Theorem 1.1.

\section{DynamiCal Stability Under SMALl PERTURBations}

In this section, we are concerned with the large-time asymptotic stability of the steady solution $F_{*}$ obtained in Theorem 1.1. For this purpose, we introduce the perturbation

$$
f(t, x, v):=\frac{F(t, x, v)-F_{*}(x, v)}{\sqrt{\mu}} .
$$

Then the initial-boundary value problem on $f(t, x, v)$ reads as

$$
\left\{\begin{array}{l}
\partial_{t} f+v \cdot \nabla_{x} f+L f=-L_{\sqrt{\mu} f_{*}} f+\Gamma(f, f), \\
\left.f(t, x, v)\right|_{t=0}=f_{0}(x, v):=\frac{F(0, x, v)-F_{*}(x, v)}{\mu^{\frac{1}{2}}(v)} \\
\left.f(t, x, v)\right|_{\gamma_{-}}=P_{\gamma} f+\frac{\mu_{\theta}-\mu}{\sqrt{\mu}} \int_{n(x) \cdot u>0} f(t, x, u) \sqrt{\mu(u)}|n(x) \cdot u| \mathrm{d} u .
\end{array}\right.
$$

Here $P_{\gamma} f$ is defined in (3.2), the linearized collision operator $L$ is defined in (2.1), the nonlinear term $\Gamma(f, f)$ is defined in (2.2) and

$$
L_{\sqrt{\mu} f_{*}} f:=-\frac{1}{\sqrt{\mu}}\left[Q\left(\sqrt{\mu} f_{*}, \sqrt{\mu} f\right)+Q\left(\sqrt{\mu} f, \sqrt{\mu} f_{*}\right)\right] .
$$

Recall (1.9). Let

$$
h(t, x, v):=w(v) f(t, x, v) .
$$

Then one can reformulate (4.1) as

$$
\left\{\begin{array}{l}
\partial_{t} h+v \cdot \nabla_{x} h+\nu_{*}(x, v) h-K_{w} h=-w L_{\sqrt{\mu} f_{*}} f+w \Gamma(f, f), \\
\left.h\right|_{t=0}=w f_{0}:=h_{0}, \\
\left.h\right|_{\gamma_{-}}=\frac{1}{\tilde{w}(v)} \int_{n(x) \cdot u>0} h(u) \tilde{w}(u) \mathrm{d} \sigma(x)+w(v) \frac{\mu_{\theta}-\mu}{\sqrt{\mu}} \int_{n(x) \cdot u>0} h(u) \tilde{w}(u) \mathrm{d} \sigma(x),
\end{array}\right.
$$

where $\tilde{w}$ and $K_{w} h$ are the same as ones defined before, and for each $x \in \partial \Omega, \mathrm{d} \sigma(x)$ denotes the probability measure

$$
\mathrm{d} \sigma(x)=\{n(x) \cdot u\} \mathrm{d} u
$$


in the velocity space $\mathcal{V}(x):=\left\{u \in \mathbb{R}^{3}: n(x) \cdot u>0\right\}$. Also, we have denoted the collision frequency $\nu_{*}(x, v)$ at the stationary solution as

$$
\nu_{*}(x, v):=\int_{\mathbb{R}^{3}} \int_{\mathbb{S}^{2}} B(|v-u|, \omega) F_{*}(x, u) \mathrm{d} u .
$$

For simplicity, to the end we denote

$$
r[h](t, x, v):=\frac{\mu_{\theta}-\mu}{\sqrt{\mu}} \int_{n(x) \cdot u>0} h(t, x, v) \tilde{w}(u) \mathrm{d} \sigma(x) .
$$

4.1. Characteristics for time-dependent problem. Given $(t, x, v)$, let $[X(s), V(s)]$ be the backward bi-characteristics associated with the initial-boundary value problem (4.1) on the Boltzmann equation, which is determined by

$$
\left\{\begin{array}{l}
\frac{\mathrm{d} X(s)}{\mathrm{d} s}=V(s), \frac{\mathrm{d} V(s)}{\mathrm{d} s}=0 \\
{[X(t), V(t)]=[x, v]}
\end{array}\right.
$$

The solution is then given by

$$
[X(s), V(s)]=[X(s ; t, x, v), V(s ; t, x, v)]=[x-(t-s) v, v] .
$$

Similarly as in the steady case, for each $(x, v)$ with $x \in \bar{\Omega}$ and $v \neq 0$, we define its backward exit time $t_{\mathbf{b}}(x, v) \geq 0$ to be the last moment at which the back-time straight line $[X(-\tau ; 0, x, v), V(-\tau ; 0, x, v)]$ remains in $\bar{\Omega}$ :

We also define

$$
t_{\mathbf{b}}(x, v)=\sup \{s \geq 0: x-\tau v \in \bar{\Omega} \text { for } 0 \leq \tau \leq s\}
$$

$$
x_{\mathbf{b}}(x, v)=x\left(t_{\mathbf{b}}\right)=x-t_{\mathbf{b}} v \in \partial \Omega .
$$

Let $x \in \bar{\Omega},(x, v) \notin \gamma_{0} \cup \gamma_{-}$and $\left(t_{0}, x_{0}, v_{0}\right)=(t, x, v)$. For $v_{k+1} \in \mathcal{V}_{k+1}:=\left\{v_{k+1} \cdot n\left(x_{k+1}\right)>0\right\}$, the back-time cycle is defined as

$$
\left\{\begin{array}{l}
X_{c l}(s ; t, x, v)=\sum_{k} \mathbf{1}_{\left[t_{k+1}, t_{k}\right)}(s)\left\{x_{k}-v_{k}\left(t_{k}-s\right)\right\} \\
V_{c l}(s ; t, x, v)=\sum_{k} \mathbf{1}_{\left[t_{k+1}, t_{k}\right)}(s) v_{k}
\end{array}\right.
$$

with

$$
\left(t_{k+1}, x_{k+1}, v_{k+1}\right)=\left(t_{k}-t_{\mathbf{b}}\left(x_{k}, v_{k}\right), x_{\mathbf{b}}\left(x_{k}, v_{k}\right), v_{k+1}\right) .
$$

For $k \geq 2$, the iterated integral means that

$$
\int_{\Pi_{l=1}^{k-1} \mathcal{V}_{l}} \Pi_{l=1}^{k-1} \mathrm{~d} \sigma_{l}:=\int_{\mathcal{V}_{1}} \ldots\left\{\int_{\mathcal{V}_{k-1}} \mathrm{~d} \sigma_{k-1}\right\} \cdots \mathrm{d} \sigma_{1} .
$$

where

$$
\mathrm{d} \sigma_{i}:=\mu\left(v_{i}\right)\left\{n\left(x_{i}\right) \cdot v_{i}\right\} \mathrm{d} v_{i} .
$$

Note that all $v_{l}(l=1,2, \cdots)$ are independent variables, and $t_{k}, x_{k}$ depend on $t_{l}, x_{l}, v_{l}$ for $l \leq k-1$, and the velocity space $\mathcal{V}_{l}$ implicitly depends on $\left(t, x, v, v_{1}, v_{2}, \cdots, v_{l-1}\right)$.

Define the near-grazing set of $\gamma_{+}$and $\gamma_{-}$as

$$
\gamma_{ \pm}^{\varepsilon}=\left\{(x, v) \in \gamma_{ \pm}:|v \cdot n(x)|<\varepsilon \text { or }|v| \geq \varepsilon \text { or }|v| \leq \frac{1}{\varepsilon}\right\}
$$

Then we have 
Lemma 4.1 ([13, 29]). Let $\varepsilon>0$ be a small positive constant, then it holds that

$$
\left|f \mathbf{1}_{\gamma_{ \pm} \backslash \gamma_{ \pm}^{\varepsilon}}\right|_{L^{1}(\gamma)} \leq C_{\varepsilon, \Omega}\left\{\|f\|_{L^{1}}+\left\|v \cdot \nabla_{x} f\right\|_{L^{1}}\right\},
$$

and

$$
\int_{0}^{t}\left|f(\tau) \mathbf{1}_{\gamma_{+} \backslash \gamma_{+}^{\varepsilon}}\right|_{L^{1}(\gamma)} \mathrm{d} \tau \leq C_{\varepsilon, \Omega}\left\{\|f(0)\|_{L^{1}}+\int_{0}^{t}\left[\|f(\tau)\|_{L^{1}}+\left\|\left[\partial_{\tau}+v \cdot \nabla_{x}\right] f(\tau)\right\|_{L^{1}}\right] \mathrm{d} s\right\},
$$

where the positive constant $C_{\varepsilon}>0$ depends only on $\varepsilon$.

4.2. Linear problem. In this part, we study the following linear inhomogeneous problem:

$$
\left\{\begin{array}{l}
\partial_{t} f+v \cdot \nabla_{x} f+L f=g, \\
\left.f\right|_{\gamma_{-}}=P_{\gamma} f+\frac{\mu_{\theta}-\mu}{\sqrt{\mu}} \int_{n(x) \cdot u>0} f \sqrt{\mu}|n(x) \cdot u| \mathrm{d} u, \\
\left.f(t, x, v)\right|_{t=0}=f_{0}(x, v),
\end{array}\right.
$$

where $g$ is a given function. Recall that $h(t, x, v)$ defined in (4.2). Then the equation of $h$ as well as the boundary condition read:

$$
\left\{\begin{array}{l}
\partial_{t} h+v \cdot \nabla_{x} h+\nu_{*}(x, v) h-K_{w} h=w g, \\
\left.h\right|_{\gamma_{-}}=\frac{1}{\tilde{w}(v)} \int_{n(x) \cdot u>0} h(u) \tilde{w}(u) \mathrm{d} \sigma(x)+w(v) \frac{\mu_{\theta}-\mu}{\sqrt{\mu}} \int_{n(x) \cdot u>0} h(u) \tilde{w}(u) \mathrm{d} \sigma(x) .
\end{array}\right.
$$

Recall the stochastic cycle defined in (4.6). For any $0 \leq s \leq t$, we define

$$
I(t, s):=e^{-\int_{s}^{t} \nu_{*}\left(X_{c l}(\tau), V_{c l}(\tau)\right) \mathrm{d} \tau} .
$$

The following Lemma is to establish the mild formulation for (4.11). Since its proof is almost the same as for [29, Lemma 24], we omit details for brevity.

Lemma 4.2. Let $k \geq 1$ be an integer and $h(t, x, v) \in L^{\infty}$ satisfy (4.11). For any $t>0$, for almost every $(x, v) \in \bar{\Omega} \times \mathbb{R}^{3} \backslash \gamma_{0} \cup \gamma_{-}$and for any $0 \leq s \leq t$, it holds that

$$
h(t, x, v)=\sum_{n=1}^{4} \mathcal{I}_{n}+\sum_{n=5}^{14} \mathbf{1}_{\left\{t_{1}>s\right\}} \mathcal{I}_{n}
$$

where

$$
\begin{aligned}
& \mathcal{I}_{1}=\mathbf{1}_{\left\{t_{1} \leq s\right\}} I(t, s) h(s, x-(t-s) v, v) \\
& \mathcal{I}_{2}+\mathcal{I}_{3}+\mathcal{I}_{4}=\int_{\max \left\{t_{1}, s\right\}}^{t} I(t, \tau)\left[K_{w}^{m} h+K_{w}^{c} h+g\right](\tau, x-(t-\tau) v, v) \mathrm{d} \tau \\
& \mathcal{I}_{5}=\frac{I\left(t, t_{1}\right)}{\tilde{w}(v)} \int_{\prod_{j=1}^{k-1} \mathcal{V}_{j}} \sum_{l=1}^{k-1} \mathbf{1}_{\left\{t_{l+1} \leq s<t_{l}\right\}} h\left(s, x_{l}-\left(t_{l}-s\right) v_{l}, v_{l}\right) \mathrm{d} \Sigma_{l}(s) \\
& \mathcal{I}_{6}+\mathcal{I}_{7}+\mathcal{I}_{8}=\frac{I\left(t, t_{1}\right)}{\tilde{w}(v)} \int_{\prod_{j=1}^{k-1} \mathcal{V}_{j}} \int_{s}^{t_{l}} \sum_{l=1}^{k-1} \mathbf{1}_{\left\{t_{l+1} \leq s<t_{l}\right\}} \\
& \quad \times\left[K_{w}^{m} h+K_{w}^{c} h+w g\right]\left(\tau, x_{l}-\left(t_{l}-\tau\right) v_{l}, v_{l}\right) \mathrm{d} \Sigma_{l}(\tau) \mathrm{d} \tau \\
& \mathcal{I}_{9}+\mathcal{I}_{10}+\mathcal{I}_{11}=\frac{I\left(t, t_{1}\right)}{\tilde{w}(v)} \int_{\prod_{j=1}^{k-1} \mathcal{V}_{j}} \int_{t_{l+1}}^{t_{l}} \sum_{l=1}^{k-1} \mathbf{1}_{\left\{t_{l+1}>s\right\}} \\
& \times\left[K_{w}^{m} h+K_{w}^{c} h+w g\right]\left(\tau, x_{l}-\left(t_{l}-\tau\right) v_{l}, v_{l}\right) \mathrm{d} \Sigma_{l}(\tau) \mathrm{d} \tau
\end{aligned}
$$




$$
\begin{aligned}
& \mathcal{I}_{12}=I\left(t, t_{1}\right) w r\left(t_{1}, x_{1}, v\right), \quad \mathcal{I}_{13}=\frac{I\left(t, t_{1}\right)}{\tilde{w}(v)} \int_{\prod_{j=1}^{k-1} \mathcal{V}_{j}} \sum_{l=1}^{k-2} \mathbf{1}_{\left\{t_{l+1}>s\right\}} w r\left(t_{l+1}, x_{l+1}, v_{l}\right) \mathrm{d} \Sigma_{l}\left(t_{l+1}\right) \\
& \mathcal{I}_{14}=\frac{I\left(t, t_{1}\right)}{\tilde{w}(v)} \int_{\prod_{j=1}^{k-1} \mathcal{V}_{j}} \mathbf{1}_{\left\{t_{k}>s\right\}} h\left(t_{k}, x_{k}, v_{k-1}\right) \mathrm{d} \Sigma_{k-1}\left(t_{k}\right),
\end{aligned}
$$

where the measure $\mathrm{d} \Sigma_{l}(\tau)$ is defined by

$$
d \Sigma_{l}(\tau):=\left\{\prod_{j=l+1}^{k-1} \mathrm{~d} \sigma_{j}\right\}\left\{\tilde{w}\left(v_{l}\right) I\left(t_{l}, \tau\right) \mathrm{d} \sigma_{l}\right\}\left\{\prod_{j=1}^{l-1} I\left(t_{j}, t_{j+1}\right) \mathrm{d} \sigma_{j}\right\} .
$$

The same as Lemma 3.2, we have

Lemma 4.3. Let $(\eta, \zeta)$ belong to

$$
\{\zeta=2,0 \leq \eta<1 / 2\} \cup\{0 \leq \zeta<2, \eta \geq 0\} .
$$

For $T_{0}$ sufficiently large, there exist constants $\hat{C}_{3}$ and $\hat{C}_{4}$ independent of $T_{0}$ such that for $k=\hat{C}_{3} T_{0}^{\frac{5}{4}}$ and $(t, x, v) \in\left[s, s+T_{0}\right] \times \bar{\Omega} \times \mathbb{R}^{3}$, it holds that

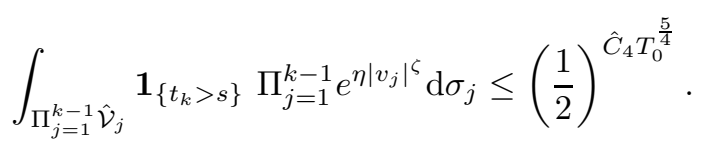

The following proposition is to clarify the solvability of the linear problem (4.10).

Proposition 4.4. Let $-3<\gamma<0, \beta>3+|\kappa|$, and $(\varpi, \zeta)$ satisfy (1.10). Assume that

$$
\int_{\Omega} \int_{\mathbb{R}^{3}} f_{0}(x, v) \mu^{\frac{1}{2}}(v) \mathrm{d} x \mathrm{~d} v=\int_{\Omega} \int_{\mathbb{R}^{3}} g(t, x, v) \mu^{\frac{1}{2}}(v) \mathrm{d} x \mathrm{~d} v=0,
$$

and also assume that

$$
\left\|w f_{0}\right\|_{L^{\infty}}+\sup _{s \geq 0} e^{\lambda_{0} s^{\alpha}}\left\|\nu^{-1} w g(s)\right\|_{L^{\infty}}<\infty
$$

where $\lambda_{0}>0$ is a small constant to be chosen in the proof. Then the linear IBVP problem (4.10) admits a unique solution $f(t, x, v)$ satisfying

$$
\sup _{0 \leq s \leq t} e^{\lambda_{0} s^{\alpha}}\left\{\|w f(s)\|_{L^{\infty}}+|w f(s)|_{L^{\infty}(\gamma)}\right\} \leq C\left\|w f_{0}\right\|_{L^{\infty}}+C \sup _{0 \leq s \leq t} e^{\lambda_{0} s^{\alpha}}\left\|\nu^{-1} w g(s)\right\|_{L^{\infty}}
$$

for any $t \geq 0$. Moreover, if $\Omega$ is convex, $f_{0}(x, v)$ is continuous except on $\gamma_{0}, g$ is continuous in $(0, \infty) \times \Omega \times \mathbb{R}^{3}$,

$$
\left.f_{0}\right|_{\gamma_{-}}=P_{\gamma} f_{0}+\frac{\mu_{\theta}-\mu}{\sqrt{\mu}} \int_{\left\{n(x) \cdot v^{\prime}>0\right\}} f_{0} \sqrt{\mu}\left|n(x) \cdot v^{\prime}\right| \mathrm{d} v^{\prime},
$$

and $\theta(x)$ is continuous on $\partial \Omega$, then $f(t, x, v)$ is continuous over $[0, \infty) \times\left\{\bar{\Omega} \times \mathbb{R}^{3} \backslash \gamma_{0}\right\}$.

The proof of Proposition 4.4 will be given after we prepare two lemmas.

Lemma 4.5. Let $h(t, x, v)$ be the $L^{\infty}$-solution of the linear problem (4.11). Then for any $s \geq 0$, for any $s \leq t \leq s+T_{0}$ with $T_{0}>0$ is sufficiently large, and for almost everywhere $(x, v) \in \bar{\Omega} \times \mathbb{R}^{3} \backslash \gamma_{0}$, it holds that

$$
\begin{aligned}
|h(t, x, v)| \leq & C T_{0}^{5 / 2} e^{-\lambda_{1}(t-s)^{\alpha}}\|h(s)\|_{L^{\infty}} \\
& +C T_{0}^{5 / 2} e^{-\tilde{\lambda} t^{\alpha}}\left\{m^{3+\kappa}+\delta+2^{-T_{0}}+\frac{1}{N}\right\} \cdot \sup _{s \leq \tau \leq t} e^{\tilde{\lambda} \tau^{\alpha}}\left\{\|h(\tau)\|_{L^{\infty}}+|h(\tau)|_{L^{\infty}(\gamma)}\right\} \\
& +C_{N, T_{0}, m} e^{-\tilde{\lambda} t^{\alpha}} \sup _{s \leq \tau \leq t}\left\|e^{\tilde{\lambda} \tau^{\alpha}} f(\tau)\right\|_{L^{2}}+C T_{0}^{5 / 2} e^{-\tilde{\lambda} t^{\alpha}} \sup _{s \leq \tau \leq t}\left\|\nu^{-1} e^{\tilde{\lambda} \tau^{\alpha}} w g(\tau)\right\|_{L^{\infty}},
\end{aligned}
$$

where $\lambda_{1}>0$ is a generic constant given in (4.22), and $0<\tilde{\lambda}<\lambda_{1}$ is an arbitrary constant to be chosen later. Here $m>0$ can be chosen arbitrarily small and $N$ can be chosen arbitrarily large. 
Proof. We take $T_{0}$ sufficiently large and $k=\hat{C}_{3} T_{0}^{5 / 4}$ such that (4.13) holds for $\eta=\frac{5}{16}$. Recall $\nu_{*}(x, v)$ defined in (4.4). Then it holds from (1.12) that

$$
\frac{\nu(v)}{2} \leq \nu_{*}(x, v) \leq \frac{3 \nu(v)}{2},
$$

provided that $\delta>0$ is suitably small. Hence it holds that

$$
e^{-\frac{3 \nu(v)}{2}(t-s)} \leq I(t, s) \leq e^{-\frac{\nu(v)}{2}(t-s)},
$$

for any $0 \leq s \leq t$. We first estimate $\mathcal{I}_{1}$. On one hand, if $s \leq t \leq s+1$, it obviously holds that

$$
\left|\mathcal{I}_{1}\right| \leq\|h(s)\|_{L^{\infty}} \leq e^{\tilde{\lambda}} e^{-\tilde{\lambda}(t-s)}\|h(s)\|_{L^{\infty}}
$$

for any $\tilde{\lambda}>0$. On the other hand, if $t>s+1$, we note that

$$
0 \leq t_{\mathbf{b}}(x, v) \leq \frac{d_{\Omega}}{|v|}
$$

where $d_{\Omega}:=\sup _{x, y \in \Omega}|x-y|$ is the diameter of the bounded domain $\Omega$. Then for $|v|>d_{\Omega}$, it holds that

$$
t_{1}-s=t-t_{\mathbf{b}}(x, v)-s>0
$$

In other words, $\mathcal{I}_{1}$ appears only when the particle velocity $|v|$ is not greater than $d_{\Omega}$, so that we have

$$
\left|\mathcal{I}_{1}\right| \leq C e^{-\frac{\nu(v)(t-s)}{2}} \mathbf{1}_{\left\{|v| \leq d_{\Omega}\right\}}\|h(s)\|_{L^{\infty}} \leq C_{\Omega} e^{-\bar{\nu}_{0}(t-s)}\|h(s)\|_{L^{\infty}},
$$

where

$$
\bar{\nu}_{0}:=\frac{\inf _{|v| \leq d_{\Omega}} \nu(v)}{2}>0
$$

depends only on $d_{\Omega}$. Collecting the estimates (4.16) and (4.17) on these two cases, we have

$$
\left|\mathcal{I}_{1}\right| \leq C_{\Omega} e^{-\bar{\nu}_{0}(t-s)}\|h(s)\|_{L^{\infty}} .
$$

For $\mathcal{I}_{4}$, we split the velocity to estimate it as

$$
\left|\mathcal{I}_{4}\right| \leq \int_{\max \left\{t_{1}, s\right\}}^{t} e^{-\frac{\nu(v)(t-\tau)}{2}} \nu(v) \cdot\left\{\mathbf{1}_{\left\{|v| \leq d_{\Omega}\right\}}+\mathbf{1}_{\left\{|v|>d_{\Omega}\right\}}\right\} \cdot\left\|\nu^{-1} w g(\tau)\right\|_{L^{\infty}} \mathrm{d} \tau .
$$

For $|v|>d_{\Omega}$, we have $\max \left\{t_{1}, s\right\} \geq t_{1} \geq t-t_{\mathbf{b}}(x, v) \geq t-1$, which implies, for any $\tilde{\lambda}>0$. that

$$
\begin{aligned}
& \int_{\max \left\{t_{1}, s\right\}}^{t} e^{-\frac{\nu(v)(t-\tau)}{2}} \nu(v) \mathbf{1}_{\left\{|v|>d_{\Omega}\right\}}\left\|\nu^{-1} w g(\tau)\right\|_{L^{\infty}} \\
& \quad \leq C \int_{\max \left\{t_{1}, s\right\}}^{t} \mathbf{1}_{\{t-1 \leq \tau \leq t\}}\left\|\nu^{-1} w g(\tau)\right\|_{L^{\infty}} \\
& \leq e^{\tilde{\lambda}} \int_{\max \left\{t_{1}, s\right\}}^{t} e^{-\tilde{\lambda}(t-\tau)} \mathbf{1}_{\{t-1 \leq \tau \leq t\}}\left\|\nu^{-1} w g(\tau)\right\|_{L^{\infty}} \mathrm{d} \tau \\
& \leq C_{\tilde{\lambda}, \alpha} e^{-\tilde{\lambda} t^{\alpha}} \cdot \sup _{s \leq \tau \leq t}\left\|e^{\tilde{\lambda} \tau^{\alpha}} \nu^{-1} w g(\tau)\right\|_{L^{\infty}} .
\end{aligned}
$$

For $|v| \leq d_{\Omega}$, we have

$$
\begin{aligned}
& \int_{\max \left\{t_{1}, s\right\}}^{t} e^{-\frac{\nu(v)(t-\tau)}{2}} \nu(v) \mathbf{1}_{\left\{|v| \leq d_{\Omega}\right\}}\left\|\nu^{-1} w g(\tau)\right\|_{L^{\infty}} \mathrm{d} \tau \\
& \leq C_{\Omega} \int_{\max \left\{t_{1}, s\right\}}^{t} e^{-\bar{\nu}_{0}(t-\tau)}\left\|\nu^{-1} w g(\tau)\right\|_{L^{\infty}} \mathrm{d} \tau \\
& \quad \leq C_{\Omega, \tilde{\lambda}, \alpha} e^{-\tilde{\lambda} t^{\alpha}} \cdot \sup _{s \leq \tau \leq t}\left\|e^{\tilde{\lambda} \tau^{\alpha}} \nu^{-1} w g(\tau)\right\|_{L^{\infty}} .
\end{aligned}
$$


Conbining these two estimates, we have

$$
\left|\mathcal{I}_{4}\right| \leq C e^{-\tilde{\lambda} t^{\alpha}} \cdot \sup _{s \leq \tau \leq t}\left\|e^{\tilde{\lambda} \tau^{\alpha}} \nu^{-1} w g(\tau)\right\|_{L^{\infty}} .
$$

Similarly, by using (2.11), it holds that

$$
\left|\mathcal{I}_{2}\right| \leq C m^{3+\kappa} e^{-\tilde{\lambda} t^{\alpha}} e^{-\frac{|v|^{2}}{16}} \sup _{s \leq \tau \leq t}\left\|e^{\tilde{\lambda} \tau^{\alpha}} h(\tau)\right\|_{L^{\infty}} .
$$

For $\mathcal{I}_{5}$, we take $\left|v_{m}\right|=\max \left\{\left|v_{1}\right|,\left|v_{2}\right|, \cdots,\left|v_{l}\right|\right\}$. By a direct computation, we have, for some positive constant $c>0$, that

$$
\begin{aligned}
& \frac{I\left(t, t_{1}\right)}{\tilde{w}(v)}\left[\prod_{j=1}^{l-1} I\left(t_{j}, t_{j+1}\right)\right] \times \tilde{w}\left(v_{l}\right) I\left(t_{l}, s\right) \\
& \quad \leq \frac{e^{-c\langle v\rangle^{\kappa}\left(t-t_{1}\right)}}{\tilde{w}(v)} \prod_{j=1}^{l-1} e^{-c\left\langle v_{j}\right\rangle^{\kappa}\left(t_{j}-t_{j+1}\right)} \cdot e^{-c\left\langle v_{l}\right\rangle^{\kappa}\left(t_{l}-s\right)} \tilde{w}\left(v_{l}\right) \\
& \quad \leq \frac{C e^{-c\langle v\rangle^{\kappa}\left(t-t_{1}\right)}}{\tilde{w}(v)} e^{-c\left\langle v_{m}\right\rangle^{\kappa}\left(t_{1}-s\right)} e^{\frac{\left|v_{m}\right|^{2}}{4}} .
\end{aligned}
$$

Here we have denoted $\langle v\rangle:=\left(1+|v|^{2}\right)^{1 / 2}$. We note the fact from Young's inequality that

$$
c\langle v\rangle^{\kappa}\left(\tau_{1}-\tau_{2}\right)+\frac{|v|^{\zeta}}{16} \geq \lambda_{1}\left(\tau_{1}-\tau_{2}\right)^{\alpha}
$$

for a generic constant $\lambda_{1}>0$ depending only on $\zeta$. Then the right-hand side of (4.21) is further bounded as

$$
C \frac{e^{-c\langle v\rangle^{\kappa}\left(t-t_{1}\right)} e^{-\frac{|v| \zeta}{16}}}{\tilde{w}(v) e^{-\frac{|v| \zeta}{16}}} e^{-c\left\langle v_{m}\right\rangle^{\kappa}\left(t_{1}-s\right)-\frac{\left|v_{m}\right| \zeta}{16}} e^{\frac{5\left|v_{m}\right|^{2}}{16}} \leq C e^{-\frac{|v|^{2}}{16}} e^{-\lambda_{1}(t-s)^{\alpha}} e^{\frac{5\left|v_{m}\right|^{2}}{16}} .
$$

Here we have used the elementary fact that $x^{\alpha}+y^{\alpha} \geq(x+y)^{\alpha}$ for $x, y \geq 0$ and $0 \leq \alpha \leq 1$. Therefore, it holds that

$$
\begin{aligned}
\left|\mathcal{I}_{5}\right| \leq & \sum_{l=1}^{k-1} \sum_{m=1}^{l} C e^{-\frac{|v|^{2}}{16}} e^{-\lambda_{1}(t-s)^{\alpha}}\|h(s)\|_{L^{\infty}} \\
& \times\left\{\int_{\prod_{j=1}^{l} \mathcal{V}_{j}} \mathbf{1}_{\left\{t_{l+1} \leq s<t_{l}\right\}} \times \mathbf{1}_{\left\{\left|v_{m}\right|=\max \left[\left|v_{1}\right|,\left|v_{2}\right|, \ldots\left|v_{l}\right|\right]\right\}} e^{\frac{5\left|v_{m}\right|^{2}}{16}} \prod_{j=1}^{l} \mathrm{~d} \sigma_{j}\right\} \\
\leq & C k^{2} e^{-\frac{|v|^{2}}{16}} e^{-\lambda_{1}(t-s)^{\alpha}}\|h(s)\|_{L^{\infty}} \cdot \sup _{j}\left|\int_{\mathcal{V}_{j}} e^{\frac{5\left|v_{j}\right|^{2}}{16}} \mathrm{~d} \sigma_{j}\right| \\
\leq & C k^{2} e^{-\frac{|v|^{2}}{16}} e^{-\lambda_{1}(t-s)^{\alpha}}\|h(s)\|_{L^{\infty}} .
\end{aligned}
$$

Similarly, we have

$$
\left|\mathcal{I}_{8}\right|+\left|\mathcal{I}_{11}\right| \leq C k^{2} e^{-\frac{|v|^{2}}{16}} e^{-\tilde{\lambda} t^{\alpha}} \cdot \sup _{s \leq \tau \leq t}\left\|e^{\tilde{\lambda} \tau^{\alpha}} \nu^{-1} w g(\tau)\right\|_{L^{\infty}}
$$

Recall $r$ defined in (4.5). Similar for obtaining (4.23), we have

$$
\left|\mathcal{I}_{12}\right|+\left|\mathcal{I}_{13}\right| \leq C \delta k^{2} e^{-\frac{|v|^{2}}{16}} e^{-\tilde{\lambda} t^{\alpha}} \cdot \sup _{s \leq \tau \leq t}\left|e^{\tilde{\lambda} \tau^{\alpha}} h(\tau)\right|_{L^{\infty}\left(\gamma_{+}\right)} .
$$

By (2.11), it holds that

$$
\left|\mathcal{I}_{6}\right|+\left|\mathcal{I}_{9}\right| \leq C k^{2} m^{3+\kappa} e^{-\frac{|v|^{2}}{16}} e^{-\tilde{\lambda} t^{\alpha}} \cdot \sup _{s \leq \tau \leq t}\left\|e^{\tilde{\lambda} \tau^{\alpha}} h(\tau)\right\|_{L^{\infty}} .
$$


For $\mathcal{I}_{14}$, we have, from (4.13), that

$$
\begin{aligned}
\left|\mathcal{I}_{14}\right| & \leq C e^{-\frac{|v|^{2}}{16}} e^{-\tilde{\lambda} t^{\alpha}} \cdot \sup _{s \leq \tau \leq t}\left|e^{\tilde{\lambda} \tau^{\alpha}} h(\tau)\right|_{L^{\infty}\left(\gamma_{-}\right)} \cdot \int_{\Pi_{j=1}^{k-1} \hat{\mathcal{V}}_{j}} \mathbf{1}_{\left\{t_{k}>s\right\}} \Pi_{j=1}^{k-1} e^{\frac{5\left|v_{j}\right|^{2}}{16}} \mathrm{~d} \sigma_{j} \\
& \leq C e^{-\frac{|v|^{2}}{16}} e^{-\tilde{\lambda} t^{\alpha}} \cdot \sup _{s \leq \tau \leq t}\left|e^{\tilde{\lambda} \tau^{\alpha}} h(\tau)\right|_{L^{\infty}\left(\gamma_{-}\right)} \cdot\left(\frac{1}{2}\right)^{\hat{C}_{4} T_{0}^{\frac{5}{4}}} .
\end{aligned}
$$

Now we consider the terms involving $K_{w}^{c}$. Similar as in (4.23), we have

$$
\begin{aligned}
\left|\mathcal{I}_{7}\right| \leq & C e^{-\frac{|v|^{2}}{16}} \sum_{l=1}^{k-1} \sum_{m=1}^{l} \int_{\Pi_{j=1}^{l-1} \mathcal{V}_{j}} \mathrm{~d} \sigma_{l-1} \cdots \mathrm{d} \sigma_{1} \\
& \times \int_{\mathcal{V}_{l}} \int_{\mathbb{R}^{3}} \int_{s}^{t_{l}} e^{-\lambda_{1}(t-\tau)^{\alpha}} 1_{\left\{t_{l+1} \leq s<t_{l}\right\}} e^{\frac{5\left|v_{m}\right|^{2}}{16}}\left|k_{w}^{c}\left(v_{l}, v^{\prime}\right) h\left(\tau, x_{l}-v_{l}\left(t_{l}-\tau\right), v^{\prime}\right)\right| \mathrm{d} \tau \mathrm{d} v^{\prime} \mathrm{d} \sigma_{l} .
\end{aligned}
$$

Then, by splitting the integral domain, we further have

$$
\begin{aligned}
\left|\mathcal{I}_{7}\right| \leq & C e^{-\frac{|v|^{2}}{16}} \sum_{l=1}^{k-1} \sum_{m=1}^{l-1} \int_{\Pi_{j=1}^{l-1} \mathcal{V}_{j}} e^{\frac{5\left|v_{m}\right|^{2}}{16}} \mathrm{~d} \sigma_{l-1} \cdots \mathrm{d} \sigma_{1} \int_{\mathcal{V}_{l} \cap\left\{\left|v_{l}\right| \geq N\right\}} \int_{\mathbb{R}^{3}} \int_{s}^{t_{l}} \Delta \mathrm{d} \tau \mathrm{d} v^{\prime} \mathrm{d} \sigma_{l} \\
& +C e^{-\frac{|v|^{2}}{16}} \sum_{l=1}^{k-1} \sum_{m=1}^{l-1} \int_{\Pi_{j=1}^{l-1} \mathcal{V}_{j}} e^{\frac{5\left|v_{m}\right|^{2}}{16}} \mathrm{~d} \sigma_{l-1} \cdots \mathrm{d} \sigma_{1} \int_{\mathcal{V}_{l} \cap\left\{\left|v_{l}\right| \leq N\right\}} \int_{\mathbb{R}^{3}} \int_{t_{l}-\frac{1}{N}}^{t_{l}} \Delta \mathrm{d} \tau \mathrm{d} v^{\prime} \mathrm{d} \sigma_{l} \\
& +C e^{-\frac{|v|^{2}}{16}} \sum_{l=1}^{k-1} \sum_{m=1}^{l-1} \int_{\Pi_{j=1}^{l-1} \mathcal{V}_{j}} e^{\frac{5\left|v_{m}\right|^{2}}{16}} \mathrm{~d} \sigma_{l-1} \cdots \mathrm{d} \sigma_{1} \int_{\mathcal{V}_{l} \cap\left\{\left|v_{l}\right| \leq N\right\}} \int_{\left\{\left|v^{\prime}\right| \geq 2 N\right\}} \int_{s}^{t_{l}-\frac{1}{N}} \Delta \mathrm{d} \tau \mathrm{d} v^{\prime} \mathrm{d} \sigma_{l} \\
& +C e^{-\frac{|v|^{2}}{16}} \sum_{l=1}^{k-1} \sum_{m=1}^{l-1} \int_{\Pi_{j=1}^{l-1} \mathcal{V}_{j}} e^{\frac{5\left|v_{m}\right|^{2}}{16}} \mathrm{~d} \sigma_{l-1} \cdots \mathrm{d} \sigma_{1} \int_{\mathcal{V}_{l} \cap\left\{\left|v_{l}\right| \leq N\right\}} \int_{\left\{\left|v^{\prime}\right| \leq 2 N\right\}} \int_{s}^{t_{l}-\frac{1}{N}} \Delta \mathrm{d} \tau \mathrm{d} v^{\prime} \mathrm{d} \sigma_{l} \\
:= & C e^{-\frac{|v|^{2}}{16}} \sum_{l=1}^{k-1}\left\{\mathcal{I}_{71 l}+\mathcal{I}_{72 l}+\mathcal{I}_{73 l}+\mathcal{I}_{74 l}\right\},
\end{aligned}
$$

where we have denoted that

$$
\Delta:=e^{-\lambda_{1}(t-\tau)^{\alpha}} \mathbf{1}_{\left\{t_{l+1} \leq s<t_{l}\right\}} e^{\frac{5\left|v_{l}\right|^{2}}{16}}\left|k_{w}^{c}\left(v_{l}, v^{\prime}\right) h\left(\tau, x_{l}-v_{l}\left(t_{l}-\tau\right), v^{\prime}\right)\right| .
$$

For $\mathcal{I}_{71 l}$, we use (2.14) to obtain, for any $0<\tilde{\lambda}<\lambda_{1}$, that

$$
\begin{aligned}
\mathcal{I}_{71 l} & \leq C k e^{-\tilde{\lambda} t^{\alpha}} \cdot \sup _{s \leq \tau \leq t}\left\|e^{\tilde{\lambda} \tau^{\alpha}} h(\tau)\right\|_{L^{\infty}} \cdot \sup _{j=0, \cdots, l-1}\left|\int_{\mathcal{V}_{j}} e^{\frac{5\left|v_{j}\right|^{2}}{16}} \mathrm{~d} \sigma_{j}\right| \cdot\left|\int_{\left|v_{l}\right| \geq N} e^{-\frac{\left|v_{l}\right|^{2}}{16}} \mathrm{~d} v_{l}\right| \\
& \leq C k e^{-\frac{N^{2}}{32}} e^{-\tilde{\lambda} t^{\alpha}} \cdot \sup _{s \leq \tau \leq t}\left\|e^{\tilde{\lambda} \tau^{\alpha}} h(\tau)\right\|_{L^{\infty}} .
\end{aligned}
$$

For $\mathcal{I}_{72 l}$, it is straightforward to see that

$$
\mathcal{I}_{72 l} \leq \frac{C k}{N} e^{-\tilde{\lambda} t^{\alpha}} \cdot \sup _{s \leq \tau \leq t}\left\|e^{\tilde{\lambda} \tau^{\alpha}} h(\tau)\right\|_{L^{\infty}} .
$$

For $\mathcal{I}_{73 l}$, since $\left|v^{\prime}-v_{l}\right| \geq N$, then by (2.14), it holds that

$$
\left|\mathcal{I}_{73 l}\right| \leq C k e^{-\frac{N^{2}}{32}} e^{-\tilde{\lambda} t^{\alpha}} \cdot \sup _{s \leq \tau \leq t}\left\|e^{\tilde{\lambda} \tau^{\alpha}} h(\tau)\right\|_{L^{\infty}} .
$$


For $\mathcal{I}_{74 l}$, by Hölder's inequality, it holds that

$$
\begin{aligned}
& \int_{\mathcal{V}_{l} \cap\left\{\left|v_{l}\right| \leq N\right\}} \int_{\left\{\left|v^{\prime}\right| \leq 2 N\right\}} \int_{s}^{t_{l}-\frac{1}{N}} \Delta \mathrm{d} \tau \mathrm{d} v^{\prime} \mathrm{d} \sigma_{l} \\
& \leq C \int_{s}^{t_{l}-\frac{1}{N}} e^{-\lambda_{1}(t-\tau)^{\alpha}} \mathrm{d} \tau\left\{\int_{\mathcal{V}_{l} \cap\left\{\left|v_{l}\right| \leq N\right\}} \int_{\left|v^{\prime}\right| \leq 2 N} e^{-\frac{1}{8}\left|v_{l}\right|^{2}}\left|k_{w}^{c}\left(v_{l}, v^{\prime}\right)\right|^{2} \mathrm{~d} v^{\prime} \mathrm{d} v_{l}\right\}^{1 / 2} \\
& \quad \times\left\{\int_{\mathcal{V}_{l} \cap\left\{\left|v_{l}\right| \leq N\right\}} \int_{\left|v^{\prime}\right| \leq 2 N} \mathbf{1}_{\left\{t_{l+1} \leq s<t_{l}\right\}}\left|h\left(\tau, x_{l}-v_{l}\left(t_{l}-\tau\right), v^{\prime}\right)\right|^{2} \mathrm{~d} v^{\prime} \mathrm{d} v_{l}\right\}^{1 / 2} \\
& \leq C_{N} m^{\kappa-1} \int_{s}^{t_{l}-\frac{1}{N}} e^{-\lambda_{1}(t-\tau)^{\alpha}} \mathrm{d} \tau \\
& \quad \times\left\{\int_{\mathcal{V}_{l} \cap\left\{\left|v_{l}\right| \leq N\right\}} \int_{\left|v^{\prime}\right| \leq 2 N} \mathbf{1}_{\left\{t_{l+1} \leq s<t_{l}\right\}}\left|f\left(\tau, x_{l}-v_{l}\left(t_{l}-\tau\right), v^{\prime}\right)\right|^{2} \mathrm{~d} v^{\prime} \mathrm{d} v_{l}\right\}^{1 / 2} .
\end{aligned}
$$

Here we have used (2.12) for $\alpha=1$ in the last inequality. Note that $y_{l}:=x_{l}-v\left(t_{l}-\tau\right) \in \Omega$ for $s \leq \tau \leq t_{l}-\frac{1}{N}$. Making change of variable $v_{l} \rightarrow y_{l}$, we obtain that

$$
\int_{\mathcal{V}_{l} \cap\left\{\left|v_{l}\right| \leq N\right\}} \int_{\mathbb{R}^{3}} \int_{s}^{t_{l}-\frac{1}{N}} \Delta \mathrm{d} \tau \mathrm{d} v^{\prime} \mathrm{d} \sigma_{l} \leq C_{N} m^{\kappa-1} e^{-\tilde{\lambda} t^{\alpha}} \cdot \sup _{s \leq \tau \leq t} e^{\tilde{\lambda} \tau^{\alpha}}\|f(\tau)\|_{L^{2}} .
$$

Combing this with (4.28), (4.29), 4.30), 4.31), we have

$$
\left|\mathcal{I}_{7}\right| \leq \frac{C k}{N} e^{-\frac{|v|^{2}}{16}} e^{-\tilde{\lambda} t^{\alpha}} \cdot \sup _{s \leq \tau \leq t}\left\|e^{\tilde{\lambda} \tau^{\alpha}} h(\tau)\right\|_{L^{\infty}}+C_{N} m^{\kappa-1} e^{-\frac{|v|^{2}}{16}} e^{-\tilde{\lambda} t^{\alpha}} \cdot \sup _{s \leq \tau \leq t} e^{\tilde{\lambda} \tau^{\alpha}}\|f(\tau)\|_{L^{2}}
$$

Similarly,

$$
\left|\mathcal{I}_{10}\right| \leq \frac{C k}{N} e^{-\frac{|v|^{2}}{16}} e^{-\tilde{\lambda} t^{\alpha}} \cdot \sup _{s \leq \tau \leq t}\left\|e^{\tilde{\lambda} \tau^{\alpha}} h(\tau)\right\|_{L^{\infty}}+C_{N} m^{\kappa-1} e^{-\frac{|v|^{2}}{16}} e^{-\tilde{\lambda} t^{\alpha}} \cdot \sup _{s \leq \tau \leq t} e^{\tilde{\lambda} \tau^{\alpha}}\|f(\tau)\|_{L^{2}} .
$$

Substituting (4.18), (4.19), (4.20), 4.23), (4.25), (4.24), (4.26), (4.27), (4.32), (4.33) into (4.12), we have

$$
|h(t, x, v)| \leq \int_{\max \left\{t_{1}, s\right\}}^{t} \int_{\mathbb{R}^{3}} I(t, \tau)\left|k_{w}^{c}(v, u) h(\tau, x-(t-\tau) v, u)\right| \mathrm{d} u \mathrm{~d} \tau+\hat{A}(t, v),
$$

where we have denoted

$$
\begin{aligned}
\hat{A}(t, v):= & C k^{2} e^{-\lambda_{1}(t-s)^{\alpha}}\|h(s)\|_{L^{\infty}} \\
& +C k^{2} e^{-\tilde{\lambda} t^{\alpha}} e^{-\frac{|v|^{2}}{16}}\left\{m^{3+\kappa}+\delta+2^{-T_{0}}+\frac{1}{N}\right\} \cdot \sup _{s \leq \tau \leq t} e^{\tilde{\lambda} \tau^{\alpha}}\left\{\|h(\tau)\|_{L^{\infty}}+|h(\tau)|_{L^{\infty}(\gamma)}\right\} \\
& +C_{N, T_{0}, m} e^{-\frac{|v|^{2}}{16}} e^{-\tilde{\lambda} t^{\alpha}} \cdot \sup _{s \leq \tau \leq t}\left\|e^{\tilde{\lambda} \tau^{\alpha}} f(\tau)\right\|_{L^{2}} \\
& +C k^{2} e^{-\tilde{\lambda} t^{\alpha}} \sup _{s \leq \tau \leq t}\left\|\nu^{-1} e^{\tilde{\lambda} \tau^{\alpha}} w g(\tau)\right\|_{L^{\infty}} .
\end{aligned}
$$

Denote $x^{\prime}:=x-(t-\tau) v$ and $t_{1}^{\prime}:=t_{1}\left(\tau, x^{\prime}, u\right)$. Now we use (4.34) for $h(\tau, x-(t-\tau) v, u)$ to evaluate

$$
\begin{aligned}
|h(t, x, v)| \leq & \hat{A}(t, v)+\int_{\max \left\{t_{1}, s\right\}}^{t} \int_{\mathbb{R}^{3}} I(t, \tau)\left|k_{w}^{c}(v, u) \hat{A}(\tau, u)\right| \mathrm{d} u \mathrm{~d} \tau \\
+ & \int_{s}^{t} \int_{s}^{\tau} \int_{\mathbb{R}^{3}} \int_{\mathbb{R}^{3}} \mathbf{1}_{\left\{\max \left\{t_{1}, s\right\} \leq \tau \leq t\right\}} \mathbf{1}_{\left\{\max \left\{t_{1}^{\prime}, s\right\} \leq \tau^{\prime} \leq \tau\right\}} \\
& \quad \times I\left(t, \tau^{\prime}\right)\left|k_{w}^{c}(v, u) k_{w}^{c}\left(u, u^{\prime}\right) h\left(\tau^{\prime}, x^{\prime}-\left(\tau-\tau^{\prime}\right) u, u^{\prime}\right)\right| \mathrm{d} u \mathrm{~d} u^{\prime} \mathrm{d} \tau^{\prime} \mathrm{d} \tau \\
:= & \hat{A}(t, v)+\hat{B}_{1}+\hat{B}_{2}
\end{aligned}
$$


Similar for obtaining (4.19), we have

$$
\begin{aligned}
\left|\hat{B}_{1}\right| \leq & \int_{\max \left\{t_{1}, s\right\}}^{t} \int_{\mathbb{R}^{3}}\left\{\mathbf{1}_{\left\{|v| \leq d_{\Omega}\right\}}+\mathbf{1}_{\left\{|v|>d_{\Omega}\right\}}\right\} I(t, \tau)\left|k_{w}(v, u) A(\tau, u)\right| \mathrm{d} u \mathrm{~d} \tau \\
\leq & C_{\Omega} \int_{\max \left\{t_{1}, s\right\}}^{t} \int_{\mathbb{R}^{3}}\left\{e^{-\bar{\nu}_{0}(t-\tau)} \mathbf{1}_{\{t-1 \leq \tau \leq t\}}+e^{-\bar{\nu}_{0}(t-\tau)} \mathbf{1}_{\left\{|v| \leq d_{\Omega}\right\}}\right\}\left|k_{w}(v, u) A(\tau, u)\right| \mathrm{d} u \mathrm{~d} \tau \\
\leq & C k^{2} e^{-\lambda_{1}(t-s)^{\alpha}}\|h(s)\|_{L^{\infty}} \\
& +C k^{2} e^{-\tilde{\lambda} t^{\alpha}}\left\{m^{3+\kappa}+\delta+2^{-T_{0}}+\frac{1}{N}\right\} \cdot \sup _{s \leq \tau \leq t} e^{\tilde{\lambda} \tau^{\alpha}}\left\{\|h(\tau)\|_{L^{\infty}}+|h(\tau)|_{L^{\infty}(\gamma)}\right\} \\
& +C_{N, T_{0}, m} e^{-\tilde{\lambda} t^{\alpha}} \cdot \sup _{s \leq \tau \leq t}\left\|e^{\tilde{\lambda} \tau^{\alpha}} f(\tau)\right\|_{L^{2}}+C k^{2} e^{-\tilde{\lambda} t^{\alpha}} \sup _{s \leq \tau \leq t}\left\|\nu^{-1} e^{\tilde{\lambda} \tau^{\alpha}} w g(\tau)\right\|_{L^{\infty}}
\end{aligned}
$$

Finally, we estimate $\hat{B}_{2}$. If $|v|>N$, we have from (2.14) that

$$
\begin{aligned}
\left|\hat{B}_{2}\right| & \leq C(1+|v|)^{-2} e^{-\tilde{\lambda} t^{\alpha}} \sup _{s \leq \tau \leq t}\left\|e^{\tilde{\lambda} \tau^{\alpha}} h(\tau)\right\|_{L^{\infty}} \\
& \leq \frac{C}{N^{2}} e^{-\tilde{\lambda} t^{\alpha}} \sup _{s \leq \tau \leq t}\left\|e^{\tilde{\lambda} \tau^{\alpha}} h(\tau)\right\|_{L^{\infty}}
\end{aligned}
$$

If $|v| \leq N$, we denote the integrand of $B_{2}$ as $U\left(\tau^{\prime}, v^{\prime}, v^{\prime \prime} ; \tau, v\right)$, and split the integral domain with respect to $\mathrm{d} \tau^{\prime} \mathrm{d} v^{\prime \prime} \mathrm{d} v^{\prime}$ into the following four parts:

$$
\begin{aligned}
\cup_{i=1}^{4} \mathcal{O}_{i}:= & \left\{\left|v^{\prime}\right| \geq 2 N\right\} \\
& \cup\left\{\left|v^{\prime}\right| \leq 2 N,\left|v^{\prime \prime}\right|>3 N\right\} \\
& \cup\left\{\left|v^{\prime}\right| \leq 2 N,\left|v^{\prime \prime}\right| \leq 3 N, \tau-\frac{1}{N} \leq \tau^{\prime} \leq \tau\right\} \\
& \cup\left\{\left|v^{\prime}\right| \leq 2 N,\left|v^{\prime \prime}\right| \leq 3 N, s \leq \tau^{\prime} \leq \tau-\frac{1}{N}\right\}
\end{aligned}
$$

Over $\mathcal{O}_{1}$ and $\mathcal{O}_{2}$, we have either $\left|v-v^{\prime}\right| \geq N$ or $\left|v^{\prime}-v^{\prime \prime}\right| \geq N$, so that one of the following is valid:

$$
\left\{\begin{array}{l}
\left|k_{w}^{c}\left(v, v^{\prime}\right)\right| \leq e^{-\frac{N^{2}}{32}} e^{\frac{\left|v-v^{\prime}\right|^{2}}{32}}\left|k_{w}^{c}\left(v, v^{\prime}\right)\right| \\
\left|k_{w}^{c}\left(v^{\prime}, v^{\prime \prime}\right)\right| \leq e^{-\frac{N^{2}}{32}} e^{\frac{\left|v^{\prime}-v^{\prime \prime}\right|^{2}}{32}}\left|k_{w}^{c}\left(v, v^{\prime}\right)\right|
\end{array}\right.
$$

By (2.14), one has

$$
\int_{s}^{t} \int_{\mathcal{O}_{1} \cup \mathcal{O}_{2}} U\left(\tau^{\prime}, v^{\prime}, v^{\prime \prime} ; \tau, v\right) \mathrm{d} v^{\prime \prime} \mathrm{d} \tau^{\prime} \mathrm{d} v^{\prime} \mathrm{d} \tau \leq C e^{-\frac{N^{2}}{32}} e^{-\tilde{\lambda} t^{\alpha}} \sup _{s \leq \tau \leq t}\left\|e^{\tilde{\lambda} \tau^{\alpha}} h(\tau)\right\|_{L^{\infty}}
$$

Over $\mathcal{O}_{3}$, it is direct to obtain

$$
\int_{s}^{t} \int_{\mathcal{O}_{3}} U\left(\tau^{\prime}, v^{\prime}, v^{\prime \prime} ; \tau, v\right) \mathrm{d} v^{\prime \prime} \mathrm{d} \tau^{\prime} \mathrm{d} v^{\prime} \mathrm{d} \tau \leq \frac{C}{N} e^{-\tilde{\lambda} t^{\alpha}} \sup _{s \leq \tau \leq t}\left\|e^{\tilde{\lambda} \tau^{\alpha}} h(\tau)\right\|_{L^{\infty}}
$$


For $\mathcal{O}_{4}$, it holds from Holder's inequality that

$$
\begin{aligned}
& \int_{\mathcal{O}_{4}} U\left(\tau^{\prime}, v^{\prime}, v^{\prime \prime} ; \tau, v\right) \mathrm{d} v^{\prime \prime} \mathrm{d} \tau^{\prime} \mathrm{d} v^{\prime} \\
& \leq C_{N} e^{-\bar{\nu}_{0}(t-\tau)}\left(\int_{\mathcal{O}_{4}} e^{-\bar{\nu}_{0}\left(\tau-\tau^{\prime}\right)}\left|k_{w}^{c}(v, u) k_{w}^{c}\left(u, u^{\prime}\right)\right|^{2} \mathrm{~d} \tau^{\prime} \mathrm{d} u \mathrm{~d} u^{\prime}\right)^{1 / 2} \\
& \quad \times\left(\int_{\mathcal{O}_{4}} e^{-\bar{\nu}_{0}\left(\tau-\tau^{\prime}\right)} \mathbf{1}_{\left\{y^{\prime} \in \Omega\right\}}\left|h\left(\tau^{\prime}, y^{\prime}, u^{\prime}\right)\right|^{2} \mathrm{~d} \tau^{\prime} \mathrm{d} u \mathrm{~d} u^{\prime}\right)^{1 / 2} \\
& \leq C_{N} m^{2(\kappa-1)} e^{-\bar{\nu}_{0}(t-\tau)}\left(\int_{\mathcal{O}_{4}} e^{-\bar{\nu}_{0}\left(\tau-\tau^{\prime}\right)} \mathbf{1}_{\left\{y^{\prime} \in \Omega\right\}}\left|f\left(\tau^{\prime}, y^{\prime}, u^{\prime}\right)\right|^{2} \mathrm{~d} \tau^{\prime} \mathrm{d} u \mathrm{~d} u^{\prime}\right)^{1 / 2}
\end{aligned}
$$

where we have denoted $y^{\prime}:=x^{\prime}-\left(\tau-\tau^{\prime}\right) u$. Making change of variable $u \rightarrow y^{\prime}$ and noting that the Jacobian $\left|\frac{\mathrm{d} y^{\prime}}{\mathrm{d} u}\right| \geq \frac{1}{N^{3}}>0$ for $s \leq \tau^{\prime}<\tau-\frac{1}{N}$, the right-hand side of (4.40) is bounded by

$$
C_{N} m^{2(\kappa-1)} e^{-\tilde{\lambda} t^{\alpha}} e^{-\frac{\bar{\nu}_{0}(t-\tau)}{2}} \cdot \sup _{s \leq \tau \leq t}\left\|e^{\tilde{\lambda} \tau^{\alpha}} f(\tau)\right\|_{L^{2}}
$$

which implies that

$$
\int_{s}^{t} \int_{\mathcal{O}_{4}} U\left(\tau^{\prime}, v^{\prime}, v^{\prime \prime} ; \tau, v\right) \mathrm{d} v^{\prime \prime} \mathrm{d} \tau^{\prime} \mathrm{d} v^{\prime} \mathrm{d} \tau \leq C_{N} m^{2(\kappa-1)} e^{-\tilde{\lambda} t^{\alpha}} \cdot \sup _{s \leq \tau \leq t}\left\|e^{\tilde{\lambda} \tau^{\alpha}} f(\tau)\right\|_{L^{2}} .
$$

Combining this with (4.37), (4.38), (4.39) yields that

$$
\left|\hat{B}_{2}\right| \leq \frac{C}{N} e^{-\tilde{\lambda} t^{\alpha}} \sup _{s \leq \tau \leq t}\left\|e^{\tilde{\lambda} \tau^{\alpha}} h(\tau)\right\|_{L^{\infty}}+C_{N} m^{2(\kappa-1)} e^{-\tilde{\lambda} t^{\alpha}} \cdot \sup _{s \leq \tau \leq t}\left\|e^{\tilde{\lambda} \tau^{\alpha}} f(\tau)\right\|_{L^{2}}
$$

Substituting (4.36) and (4.41) into (4.35), one has (4.15). The proof of Lemma 4.5 is complete.

The below lemma gives the $L^{2}$-decay of the solution.

Lemma 4.6. If

$$
\int_{\Omega} \int_{\mathbb{R}^{3}} f_{0}(x, v) \mu^{\frac{1}{2}}(v) \mathrm{d} x \mathrm{~d} v=\int_{\Omega} \int_{\mathbb{R}^{3}} g(t, x, v) \mu^{\frac{1}{2}}(v) \mathrm{d} x \mathrm{~d} v=0
$$

and $|\theta-1|_{L^{\infty}(\partial \Omega)}$ is sufficiently small, then there exists a constant $\lambda_{2}>0$ such that for any $t \geq 0$,

$$
\|f(t)\|_{L^{2}} \leq C e^{-\lambda_{2} t^{\alpha}}\left\|w f_{0}\right\|_{L^{\infty}}+\int_{0}^{t} e^{-\lambda_{2}(t-s)^{\alpha}}\left\|\nu^{-1} w g(s)\right\|_{L^{\infty}} \mathrm{d} s .
$$

Proof. We first consider the case $g \equiv 0$. Multiplying both sides of (4.10) by $f$, we have

$$
\frac{1}{2}\|f(t)\|_{L^{2}}^{2}+\frac{1}{2} \int_{0}^{t}|f(s)|_{L^{2}\left(\gamma_{+}\right)}^{2} \mathrm{~d} s+\int_{0}^{t}\langle L f(s), f(s)\rangle \mathrm{d} s=\frac{1}{2}\left\|f_{0}\right\|_{L^{2}}^{2}+\frac{1}{2} \int_{0}^{t}\left|P_{\gamma} f+r\right|_{L^{2}\left(\gamma_{-}\right)}^{2} \mathrm{~d} s,
$$

where $r$ is defined in (4.5). By the coercivity estimate (2.7), it holds that

$$
\int_{0}^{t}\langle L f(s), f(s)\rangle \mathrm{d} s \geq c_{0} \int_{0}^{t}\left\|\nu^{1 / 2}(I-P) f(s)\right\|_{L^{2}}^{2} \mathrm{~d} s .
$$

Notice that $P_{\gamma} r \equiv 0$. Therefore, it follows that

$$
\begin{aligned}
\frac{1}{2} \int_{0}^{t}\left|P_{\gamma} f+r\right|_{L^{2}\left(\gamma_{-}\right)}^{2} \mathrm{~d} s & =\frac{1}{2} \int_{0}^{t}\left|P_{\gamma} f(s)\right|_{L^{2}\left(\gamma_{+}\right)}^{2} \mathrm{~d} s+\frac{1}{2} \int_{0}^{t}|r(s)|_{L^{2}\left(\gamma_{-}\right)}^{2} \mathrm{~d} s \\
& \leq \frac{1}{2} \int_{0}^{t}\left|P_{\gamma} f(s)\right|_{L^{2}\left(\gamma_{+}\right)}^{2} \mathrm{~d} s+C \delta \int_{0}^{t}|f(s)|_{L^{2}\left(\gamma_{+}\right)}^{2} \mathrm{~d} s .
\end{aligned}
$$


To estimate $\left|P_{\gamma} f\right|_{L^{2}\left(\gamma_{+}\right)}$, recall the cutoff function $\mathbf{1}_{\gamma_{+}^{\varepsilon}}$ with respect to the near grazing set $\gamma_{+}^{\varepsilon}$ defined in (4.7). Then we have

$$
\begin{aligned}
\left|P_{\gamma} f(s)\right|_{L^{2}\left(\gamma_{+}\right)}^{2} & =\int_{\gamma_{-}} \mu(v)|n(x) \cdot v| \mathrm{d} \gamma \times\left(\int_{n(x) \cdot v^{\prime}>0} f(s)\left\{\mathbf{1}_{\gamma_{+}^{\varepsilon}}+\mathbf{1}_{\gamma_{+}^{\varepsilon} \backslash \gamma_{+}^{\varepsilon}}\right\} \sqrt{\mu}\left|n(x) \cdot v^{\prime}\right| \mathrm{d} v^{\prime}\right)^{2} \\
& \leq C \varepsilon|f(s)|_{L^{2}\left(\gamma_{+}\right)}^{2}+C\left|e^{-\frac{|v|^{2}}{16}} f(s) \mathbf{1}_{\gamma_{+} \backslash \gamma_{+}^{\varepsilon}}\right|_{L^{2}\left(\gamma_{+}\right)}^{2} .
\end{aligned}
$$

Notice that

which implies that

$$
\frac{1}{2}\left(\partial_{t}+v \cdot \nabla_{x}\right) e^{-\frac{|v|^{2}}{8}} f^{2}=e^{-\frac{|v|^{2}}{8}} f L f
$$

$$
\int_{0}^{t}\left\|\left(\partial_{t}+v \cdot \nabla_{x}\right) e^{-\frac{|v|^{2}}{8}} f^{2}(s)\right\|_{L^{1}} \mathrm{~d} s \leq C \int_{0}^{t}\left\|e^{-\frac{|v|^{2}}{16}} f(s)\right\|_{L^{2}}^{2} \mathrm{~d} s .
$$

Therefore, by the trace estimate (4.9), we have

$$
\begin{aligned}
\int_{0}^{t}\left|e^{-\frac{|v|^{2}}{16}} f(s) \mathbf{1}_{\gamma_{+} \backslash \gamma_{+}^{\varepsilon}}\right|_{L^{2}\left(\gamma_{+}\right)}^{2} \mathrm{~d} s & =\int_{0}^{t}\left|e^{-\frac{|v|^{2}}{8}} f^{2}(s) \mathbf{1}_{\gamma_{+} \backslash \gamma_{+}^{\varepsilon}}\right|_{L^{1}\left(\gamma_{+}\right)} \mathrm{d} s \\
& \leq C_{\varepsilon}\left\|e^{-\frac{|v|^{2}}{16}} f_{0}\right\|_{L^{2}}^{2}+C_{\varepsilon} \int_{0}^{t}\left\|e^{-\frac{|v|^{2}}{16}} f(s)\right\|_{L^{2}}^{2} \mathrm{~d} s \\
& \leq C_{\varepsilon}\left\|f_{0}\right\|_{L^{2}}^{2}+C_{\varepsilon} \int_{0}^{t}\left\|\nu^{1 / 2} f(s)\right\|_{L^{2}}^{2} \mathrm{~d} s .
\end{aligned}
$$

Combining this with (4.47), we have

$$
\begin{aligned}
& \int_{0}^{t}|f(s)|_{L^{2}\left(\gamma_{+}\right)}^{2} \mathrm{~d} s \\
& \quad=\int_{0}^{t}\left|P_{\gamma} f(s)\right|_{L^{2}\left(\gamma_{+}\right)}^{2} \mathrm{~d} s+\int_{0}^{t}\left|\left(I-P_{\gamma}\right) f(s)\right|_{L^{2}\left(\gamma_{+}\right)}^{2} \mathrm{~d} s \\
& \quad \leq C \int_{0}^{t}\left|\left(I-P_{\gamma}\right) f(s)\right|_{L^{2}\left(\gamma_{+}\right)}^{2} \mathrm{~d} s+C \varepsilon \int_{0}^{t}|f(s)|_{L^{2}\left(\gamma_{+}\right)}^{2} \mathrm{~d} s+C_{\varepsilon}\left\|f_{0}\right\|_{L^{2}}^{2}+C_{\varepsilon} \int_{0}^{t}\left\|\nu^{1 / 2} f(s)\right\|_{L^{2}}^{2} \mathrm{~d} s \\
& \quad \leq C \int_{0}^{t}\left|\left(I-P_{\gamma}\right) f(s)\right|_{L^{2}\left(\gamma_{+}\right)}^{2} \mathrm{~d} s+C\left\|f_{0}\right\|_{L^{2}}^{2}+C \int_{0}^{t}\left\|\nu^{1 / 2} f(s)\right\|_{L^{2}}^{2} \mathrm{~d} s .
\end{aligned}
$$

Here we have taken $\varepsilon>0$ suitably small in the last inequality. For the macroscopic part $P f$, we multiply $\sqrt{\mu}$ to both sides of the first equation in (4.10) and use (4.42) to get

$$
\int_{\Omega} \int_{\mathbb{R}^{3}} f(t, x, v) \mu^{\frac{1}{2}}(v) \mathrm{d} x \mathrm{~d} v=\int_{\Omega} \int_{\mathbb{R}^{3}} f_{0}(x, v) \mu^{\frac{1}{2}}(v) \mathrm{d} x \mathrm{~d} v=0 .
$$

Then similar as [21, Lemma 6.1], there exists a functional $\mathfrak{e}_{f}(t)$ with $\left|\mathfrak{e}_{f}(t)\right| \lesssim\|f(t)\|_{L^{2}}^{2}$ such that

$$
\begin{aligned}
\int_{0}^{t}\left\|\nu^{1 / 2} P f(s)\right\|_{L^{2}}^{2} \mathrm{~d} s \lesssim & \left(\mathfrak{e}_{f}(t)-\mathfrak{e}_{f}(0)\right)+\int_{0}^{t}\left\|\nu^{1 / 2}(I-P) f(s)\right\|_{L^{2}}^{2} \mathrm{~d} s \\
& \quad+\int_{0}^{t}|r(s)|_{L^{2}\left(\gamma_{-}\right)}^{2} \mathrm{~d} s+\int_{0}^{t}\left|\left(I-P_{\gamma}\right) f(s)\right|_{L^{2}\left(\gamma_{+}\right)}^{2} \mathrm{~d} s . \\
\lesssim & \left(\mathfrak{e}_{f}(t)-\mathfrak{e}_{f}(0)\right)+\int_{0}^{t}\left\|\nu^{1 / 2}(I-P) f(s)\right\|_{L^{2}}^{2} \mathrm{~d} s \\
& +\delta \int_{0}^{t}|f(s)|_{L^{2}\left(\gamma_{+}\right)}^{2} \mathrm{~d} s+\int_{0}^{t}\left|\left(I-P_{\gamma}\right) f(s)\right|_{L^{2}\left(\gamma_{+}\right)}^{2} \mathrm{~d} s .
\end{aligned}
$$


Suitably combining the estimate above with (4.44), (4.45), (4.46) and (4.48) and taking $\delta>0$ suitably small, we have

$$
\|f(t)\|_{L^{2}}^{2}+\int_{0}^{t}\left\|\nu^{1 / 2} f(s)\right\|_{L^{2}}^{2} \mathrm{~d} s+\int_{0}^{t}|f(s)|_{L^{2}\left(\gamma_{+}\right)}^{2} \mathrm{~d} s \leq C\left\|f_{0}\right\|_{L^{2}}^{2}
$$

Next we need to obtain the weighted $L^{2}$ estimate in order to obtain $L^{2}$ decay of $f$. Multiplying $e^{\frac{w|v| \zeta}{2}} f$ to both sides of the first equation in (4.10), we have

$$
\begin{gathered}
\frac{1}{2}\left\|e^{\frac{\varpi|\cdot| \zeta}{4}} f(t)\right\|_{L^{2}}^{2}+\frac{1}{2} \int_{0}^{t}\left|e^{\frac{\varpi|\cdot| \zeta}{4}} f(s)\right|_{L^{2}\left(\gamma_{+}\right)}^{2} \mathrm{~d} s+\int_{0}^{t}\left\|\nu^{1 / 2} e^{\frac{\varpi|\cdot| \zeta}{4}} f(s)\right\|_{L^{2}}^{2} \mathrm{~d} s \\
=\frac{1}{2}\left\|e^{\frac{\varpi|\cdot| \zeta}{4}} f_{0}\right\|_{L^{2}}^{2}+\frac{1}{2} \int_{0}^{t}\left|e^{\frac{\varpi|\cdot| \zeta}{4}} f(s)\right|_{L^{2}\left(\gamma_{-}\right)}^{2}+\int_{0}^{t}\left\langle K f(s), e^{\frac{\varpi|\cdot| \zeta}{2}} f(s)\right\rangle \mathrm{d} s .
\end{gathered}
$$

A direct computation shows that

$$
\begin{aligned}
\int_{0}^{t}\left|e^{\frac{\varpi|\cdot| \zeta}{4}} f(s)\right|_{L^{2}\left(\gamma_{-}\right)}^{2} & \leq C \int_{0}^{t}\left|e^{\frac{\varpi|\cdot| \zeta}{4}} P_{\gamma} f(s)\right|_{L^{2}\left(\gamma_{-}\right)}^{2} \mathrm{~d} s+C \int_{0}^{t}\left|e^{\frac{\varpi|\cdot| \zeta}{4}} r(s)\right|_{L^{2}\left(\gamma_{-}\right)}^{2} \mathrm{~d} s \\
& \leq C \int_{0}^{t}|f(s)|_{L^{2}\left(\gamma_{+}\right)}^{2} \mathrm{~d} s .
\end{aligned}
$$

As for the last term on the right-hand side of (4.50), we use (2.15) to obtain

$$
\int_{0}^{t}\left|\left\langle K f(s), e^{\frac{m|\cdot| \zeta}{2}} f(s)\right\rangle\right| \mathrm{d} s \leq \eta \int_{0}^{t}\left\|\nu^{1 / 2} e^{\frac{m|\cdot| \zeta}{4}} f(s)\right\|_{L^{2}}^{2} \mathrm{~d} s+C_{\eta} \int_{0}^{t}\left\|\nu^{1 / 2} f(s)\right\|_{L^{2}}^{2} \mathrm{~d} s .
$$

Therefore, suitably combining (4.49), (4.50), (4.51) and (4.52) and taking $\eta>0$ suitably small, we have

$$
\left\|e^{\frac{\varpi|\cdot| \zeta}{4}} f(t)\right\|_{L^{2}}^{2}+\int_{0}^{t}\left\|e^{\frac{\varpi|\cdot| \zeta}{4}} \nu^{1 / 2} f(s)\right\|_{L^{2}}^{2} \mathrm{~d} s+\int_{0}^{t}\left|e^{\frac{\varpi|\cdot| \zeta}{4}} f(s)\right|_{L^{2}\left(\gamma_{+}\right)}^{2} \mathrm{~d} s \leq C\left\|e^{\frac{\varpi|\cdot| \zeta}{4}} f_{0}\right\|_{L^{2}}^{2} .
$$

Now we are ready for obtaining $L^{2}$ decay of $f$. Let $\tilde{f}=e^{\lambda^{\prime}(1+t)^{\alpha}} f$, with $\lambda^{\prime}>0$ is a suitably small constant to be determined later. Then applying the same energy estimate for obtaining (4.49), we have

$$
\|\tilde{f}(t)\|_{L^{2}}^{2}+\int_{0}^{t}\left\|\nu^{1 / 2} \tilde{f}(s)\right\|_{L^{2}}^{2} \mathrm{~d} s \leq C\left\|f_{0}\right\|_{L^{2}}^{2}+C \lambda^{\prime} \int_{0}^{t}(s+1)^{\alpha-1}\|\tilde{f}(s)\|_{L^{2}}^{2} \mathrm{~d} s
$$

To estimate the last term, we split the $v$-integration domain into

$$
\mathbb{R}_{v}^{3}=\left\{1+|v| \geq(1+t)^{\frac{1}{\zeta+|\kappa|}}\right\} \cup\left\{1+|v|<(1+t)^{\frac{1}{\zeta+|\kappa|}}\right\}=\mathcal{M}(t) \cup \mathcal{M}^{c}(t) .
$$

One one hand, we have

$$
\begin{aligned}
\int_{0}^{t}(s+1)^{\alpha-1}\left\|\tilde{f} \mathbf{1}_{\mathcal{M}(s)}(s)\right\|_{L^{2}}^{2} \mathrm{~d} s & \leq C \int_{0}^{t}\left\|e^{\lambda^{\prime}(1+|\cdot|)^{\zeta}} f(s)\right\|_{L^{2}}^{2} \mathrm{~d} s \leq C \int_{0}^{t}\left\|\nu^{1 / 2} e^{\frac{\varpi 1 \cdot \mid \zeta}{4}} f(s)\right\|_{L^{2}}^{2} \mathrm{~d} s \\
& \leq C\left\|e^{\frac{\varpi|\cdot| \zeta}{4}} f_{0}\right\|_{L^{2}}^{2},
\end{aligned}
$$

by taking $\lambda^{\prime}>0$ suitably small. On the other hand, in $\mathcal{M}^{c}(s)$, we have

$$
(1+s)^{\alpha-1} \leq(1+|v|)^{(\alpha-1) \cdot(\zeta+|\kappa|)} \mathbf{1}_{\mathcal{M}^{c}(s)} \leq C \nu(v) \mathbf{1}_{\mathcal{M}^{c}(s)} .
$$

Therefore, we have

$$
\int_{0}^{t}(s+1)^{\alpha-1}\left\|\tilde{f} \mathbf{1}_{\mathcal{M}^{c}(s)}(s)\right\|_{L^{2}}^{2} \mathrm{~d} s \leq C \int_{0}^{t}\left\|\nu^{1 / 2} \tilde{f}(s)\right\|_{L^{2}}^{2} \mathrm{~d} s .
$$


Combining these estimates with (4.53) and taking $\lambda^{\prime}>0$ suitably small, we have

$$
\begin{aligned}
\|\tilde{f}(t)\|_{L^{2}}^{2}+\int_{0}^{t}\left\|\nu^{1 / 2} \tilde{f}(s)\right\|_{L^{2}}^{2} \mathrm{~d} s & \leq C\left\|e^{\frac{\varpi|\cdot| \zeta}{4}} f_{0}\right\|_{L^{2}}^{2}+C \lambda^{\prime} \int_{0}^{t}\left\|\nu^{1 / 2} \tilde{f}(s)\right\|_{L^{2}}^{2} \mathrm{~d} s \\
& \leq C\left\|e^{\frac{\varpi|\cdot| \zeta}{4}} f_{0}\right\|_{L^{2}}^{2} .
\end{aligned}
$$

Then (4.43) for $g \equiv 0$ naturally follows. We denote $G(t)$ as the solution operator to the linear homogeneous problem (4.10) with $g \equiv 0$. Then for non-trivial $g$, from Duhamel's formula, it holds that

$$
\begin{aligned}
\|f(t)\|_{L^{2}} & \leq\left\|G(t) f_{0}\right\|_{L^{2}}+\int_{0}^{t}\|G(t-s) g(s)\|_{L^{2}} \mathrm{~d} s \\
& \leq C e^{-\lambda^{\prime} t^{\alpha}}\left\|e^{\frac{\varpi|\cdot| \zeta}{4}} f_{0}\right\|_{L^{2}}+C \int_{0}^{t} e^{-\lambda^{\prime}(t-s)^{\alpha}}\left\|e^{\frac{\varpi|\cdot| \zeta \zeta}{4}} g(s)\right\|_{L^{2}} \mathrm{~d} s \\
& \leq C e^{-\lambda^{\prime} t^{\alpha}}\left\|w f_{0}\right\|_{L^{\infty}}+\int_{0}^{t} e^{-\lambda^{\prime}(t-s)^{\alpha}}\left\|\nu^{-1} w g(s)\right\|_{L^{\infty}} \mathrm{d} s .
\end{aligned}
$$

Here we have used (4.54) in the second inequality. Then (4.43) follows from (4.55) by taking $\lambda_{2}=\lambda^{\prime}$. The proof of Lemma 4.6 is complete.

Proof of Proposition 4.4: The local existence and uniqueness of solutions to the linear inhomogeneous problem (4.11) can be obtained in a similar way as in Proposition 6.2. We omit the details for brevity. In what follows we will show the decay estimate (4.14). Recall the finite-time estimate (4.15). We define $\lambda_{0}=\min \left\{\frac{\lambda_{1}}{4}, \frac{\lambda_{2}}{4}\right\}$, and

$$
m=\left(\frac{\eta}{2 C}\right)^{\frac{1}{3+\kappa}} T_{0}^{-\frac{5}{2(3+\kappa)}}
$$

with $\eta>0$ suitably small to be determined later. Then we choose $T_{0}$ suitably large and $\delta>0$ suitably small, and also take $N$ suitably large, such that

$$
C T_{0}^{5 / 2}\left\{m^{3+\kappa}+\delta+2^{-T_{0}}+\frac{1}{N}\right\} \leq \eta, \quad C T_{0}^{5 / 2} e^{-\frac{\lambda_{1}}{2} T_{0}^{\alpha}} \leq 1
$$

Then it holds from (4.15) with the choice of $\tilde{\lambda}=\lambda_{0}$ that for any $s \geq 0$ and any $t \in\left[s, s+T_{0}\right]$,

$$
\|h(t)\|_{L^{\infty}}+|h(t)|_{L^{\infty}(\gamma)} \leq e^{-\frac{\lambda_{1}}{2}(t-s)^{\alpha}}\|h(s)\|_{L^{\infty}}+e^{-\lambda_{0} t^{\alpha}} \mathcal{D}(t, s),
$$

where we have defined

$$
\begin{aligned}
\mathcal{D}(t, s)= & \eta \sup _{s \leq \tau \leq t} e^{\lambda_{0} \tau^{\alpha}}\left\{\|h(\tau)\|_{L^{\infty}}+|h(\tau)|_{L^{\infty}(\gamma)}\right\} \\
& +C \sup _{s \leq \tau \leq t}\left\|e^{\lambda_{0} \tau^{\alpha}} f(\tau)\right\|_{L^{2}}+C \sup _{s \leq \tau \leq t}\left\|\nu^{-1} e^{\lambda_{0} \tau^{\alpha}} w g(\tau)\right\|_{L^{\infty}} .
\end{aligned}
$$


For any $t>0$, there exists a positive integer $n \geq 1$, such that $n T_{0} \leq t<(n+1) T_{0}$. Then applying (4.56) to $\left[0, T_{0}\right],\left[T_{0}, 2 T_{0}\right], \cdots,\left[(n-1) T_{0}, n T_{0}\right]$ inductively, we have

$$
\begin{aligned}
\left\|h\left(n T_{0}\right)\right\|_{L^{\infty}} \leq & e^{-\frac{\lambda_{1}}{2} T_{0}^{\alpha}}\left\|h(n-1) T_{0}\right\|_{L^{\infty}}+e^{-\lambda_{0}\left(n T_{0}\right)^{\alpha}} D\left(n T_{0},[n-1] T_{0}\right) \\
\leq & e^{-\lambda_{1} T_{0}^{\alpha}}\left\|h(n-2) T_{0}\right\|_{L^{\infty}}+e^{-\lambda_{0}\left(n T_{0}\right)^{\alpha}} D\left(n T_{0},[n-1] T_{0}\right) \\
& +e^{-\frac{\lambda_{1} T_{0}^{\alpha}}{2}-\lambda_{0}\left([n-1] T_{0}\right)^{\alpha}} D\left([n-1] T_{0},[n-2] T_{0}\right) \\
\leq & e^{-\lambda_{1} T_{0}^{\alpha}}\left\|h(n-2) T_{0}\right\|_{L^{\infty}}+e^{-\lambda_{0}\left(n T_{0}\right)^{\alpha}}\left\{1+e^{-\frac{\lambda_{1} T_{0}^{\alpha}}{4}}\right\} D\left(n T_{0},[n-2] T_{0}\right) \\
\leq & \cdots \\
\leq & e^{-\frac{\lambda_{1} n T_{0}^{\alpha}}{2}}\left\|h_{0}\right\|_{L^{\infty}}+e^{-\lambda_{0}\left(n T_{0}\right)^{\alpha}}\left\{1+e^{-\frac{\lambda_{1} T_{0}^{\alpha}}{4}}+\cdots+e^{-\frac{(n-1) \lambda_{1} T_{0}^{\alpha}}{4}}\right\} \mathcal{D}\left(n T_{0}, 0\right) \\
\leq & C e^{-\frac{\lambda_{1}\left(n T_{0}\right)^{\alpha}}{2}}\left\|h_{0}\right\|_{L^{\infty}}+C e^{-\lambda_{0}\left(n T_{0}\right)^{\alpha}} \mathcal{D}\left(n T_{0}, 0\right),
\end{aligned}
$$

where in the third inequality we have used $0<\lambda_{0} \leq \frac{\lambda_{1}}{4}$. Here we also have used the elementary fact that $x^{\alpha}+y^{\alpha} \geq(x+y)^{\alpha}$ for $x, y \geq 0$ and $0 \leq \alpha \leq 1$. Finally applying (4.56) in $\left[n T_{0},(n+1) T_{0}\right]$ and using (4.58), we have

$$
\begin{aligned}
& \|h(t)\|_{L^{\infty}}+|h(t)|_{L^{\infty}(\gamma)} \\
& \leq e^{-\frac{\lambda_{1}\left(t-n T_{0}\right)^{\alpha}}{2}}\left\|h\left(n T_{0}\right)\right\|_{L^{\infty}}+e^{-\lambda_{0} t^{\alpha}} \mathcal{D}\left(t, n T_{0}\right) \\
& \leq e^{-\lambda_{0} t^{\alpha}} \mathcal{D}\left(t, n T_{0}\right)+e^{-\frac{\lambda_{1}\left(t-n T_{0}\right)^{\alpha}}{2}}\left\{C e^{-\frac{\lambda_{1}\left(n T_{0}\right)^{\alpha}}{2}}\left\|h_{0}\right\|_{L^{\infty}}+C e^{-\lambda_{0}\left(n T_{0}\right)^{\alpha}} D\left(n T_{0}, 0\right)\right\} \\
& \leq C e^{-\frac{\lambda_{1} t^{\alpha}}{2}}\left\|h_{0}\right\|_{L^{\infty}}+C e^{-\lambda_{0} t^{\alpha}} \mathcal{D}(t, 0) .
\end{aligned}
$$

Recall (4.57). Let $\eta>0$ be suitably small, then (4.14) follows from (4.59) and (4.43). Therefore, the proof of Proposition 4.4 is complete.

4.3. Proof of Theorem 1.2. The local existence and uniqueness of the solution to nonlinear problem (4.1) is provided in Proposition 6.2 In what follows we will show (1.17). Notice that for any $t>0$, it holds that

$$
\int_{\Omega} \int_{\mathbb{R}^{3}} L_{\sqrt{\mu} f_{*}} f \mathrm{~d} x \mathrm{~d} v=\int_{\Omega} \int_{\mathbb{R}^{3}} \Gamma(f, f) \mathrm{d} x \mathrm{~d} v \equiv 0 .
$$

Then applying the linear theory Proposition 4.4 to $f$, we have

$$
\begin{aligned}
& \sup _{0 \leq s \leq t} e^{\lambda_{0} s^{\alpha}}\left\{\|w f(s)\|_{L^{\infty}}+|w f(s)|_{L^{\infty}(\gamma)}\right\} \\
& \quad \leq C\left\|w f_{0}\right\|_{L^{\infty}}+C \sup _{0 \leq s \leq t} e^{\lambda_{0} s^{\alpha}}\left\{\left\|\nu^{-1} w L_{\sqrt{\mu} f_{*}} f(s)\right\|_{L^{\infty}}+\left\|\nu^{-1} w \Gamma(f, f)(s)\right\|_{L^{\infty}}\right\} \\
& \quad \leq C\left\|w f_{0}\right\|_{L^{\infty}}+C\left\{\delta+\sup _{0 \leq s \leq t}\|w f(s)\|_{L^{\infty}}\right\} \cdot \sup _{0 \leq s \leq t} e^{\lambda_{0} s^{\alpha}}\|w f(s)\|_{L^{\infty}},
\end{aligned}
$$

where we have used the nonlinear estimate (2.16). Now we make the a priori assumption that

$$
\sup _{0 \leq s \leq t} e^{\lambda_{0} s^{\alpha}}\|w f(s)\|_{L^{\infty}} \leq 2 C\left\|w f_{0}\right\|_{L^{\infty}} .
$$

Then from (4.60), we have

$$
\begin{aligned}
& \sup _{0 \leq s \leq t} e^{\lambda_{0} s^{\alpha}}\left\{\|w f(s)\|_{L^{\infty}}+|w f(s)|_{L^{\infty}(\gamma)}\right\} \\
& \quad \leq C\left\|w f_{0}\right\|_{L^{\infty}}+2 C^{2}\left\|w f_{0}\right\|_{L^{\infty}} \cdot\left\{\delta+2 C\left\|w f_{0}\right\|_{L^{\infty}}\right\} \leq \frac{3 C}{2}\left\|w f_{0}\right\|,
\end{aligned}
$$


provided that both $\delta>0$ and $\left\|w f_{0}\right\|_{L^{\infty}}$ are suitably small. This justifies that the a priori assumption can be closed. Then from a standard continuity argument, the global existence together with the estimate (1.17) follow. Therefore, the proof of Theorem 1.2 is complete.

\section{Dynamical Stability under a Class of LARge PERTURBATIONS}

The section is devoted to proving Theorem 1.3 Recall $h(t, x, v):=w f(t, x, v)$. In what follows, we make the following a priori assumption:

$$
\sup _{0 \leq s \leq T}\|h(s)\|_{L^{\infty}}+\sup _{0 \leq s \leq T}|h(s)|_{L^{\infty}(\gamma)} \leq \bar{M},
$$

where $T>0$ is an arbitrary constant and $\bar{M}$ is a positive constant depending only on $M_{0}$ as given in (1.21). We emphasize here that $M_{0}$ is not necessarily small and will be determined at the end of the proof.

5.1. $L_{x, v}^{p}$ estimates. First of all, we have the following

Lemma $5.1([30])$. Let $1<p<\infty$. Assume that $f, \partial_{t}+v \cdot \nabla_{x} f \in L^{p}\left([0, T] ; L^{p}\right)$ and $f \mathbf{1}_{\gamma_{-}} \in$ $L^{p}\left([0, T] ; L^{p}(\gamma)\right)$. Then $f \in C^{0}\left([0, T] ; L^{p}\right)$ and $f \mathbf{1}_{\gamma_{+}} \in L^{p}\left([0, T] ; L^{p}(\gamma)\right)$ and for almost every $t \in[0, T]:$

$$
\|f(t)\|_{L^{p}}^{p}+\int_{0}^{t}|f(s)|_{L^{p}\left(\gamma_{+}\right)}^{p}=\left\|f_{0}\right\|_{L^{p}}^{p}+\int_{0}^{t}|f(s)|_{L^{p}\left(\gamma_{-}\right)}^{p}+p \int_{0}^{t} \int_{\Omega} \int_{\mathbb{R}^{3}}\left\{\partial_{t} f+v \cdot \partial_{x} f\right\}|f|^{p-2} f .
$$

Moreover, we prove that the $L^{p}$ bound of solutions grows in time exponentially related to $\bar{M}$. Note that $\bar{M}$ is to be chosen depending only on $M_{0}$, so that within a finite time interval, the solution can be uniformly small in $L^{p}$ if it is so initially.

Lemma 5.2. Let $1<p<\infty$. Under the assumption (5.1), it holds that

$$
\|f(t)\|_{L^{p}} \leq e^{C_{3} \bar{M} t}\left\|f_{0}\right\|_{L^{p}}
$$

for any $t \in[0, T]$. Here $C_{3}>1$ is a generic constant depending only on $\kappa$ and $p$.

Proof. By Green's identity (5.2), one has

$$
\begin{aligned}
\|f(t)\|_{L^{p}}^{p}+\int_{0}^{t}|f(s)|_{L^{p}\left(\gamma_{+}\right)}^{p} \mathrm{~d} s+p \int_{0}^{t}\left\|\nu^{1 / p} f(s)\right\|_{L^{p}}^{p} \mathrm{~d} s \\
=\left\|f_{0}\right\|_{L^{p}}^{p}+\int_{0}^{t}|f(s)|_{L^{p}\left(\gamma_{-}\right)}^{p}+p \int_{0}^{t}\left\langle|f(s)|^{p-2} f(s), K f(s)\right\rangle \mathrm{d} s \\
\quad+p \int_{0}^{t}\left\langle|f(s)|^{p-2} f(s),-L_{\sqrt{\mu} f_{*}} f(s)+\Gamma(f, f)(s)\right\rangle \mathrm{d} s .
\end{aligned}
$$

It is straightforward to see from (2.9) that $K$ is bounded from $L^{p}$ to $L^{p}$. Therefore, one has

$$
\left|\int_{0}^{t}\left\langle|f(s)|^{p-2} f(s), K f(s)\right\rangle \mathrm{d} s\right| \leq C \int_{0}^{t}\|f(s)\|_{L^{p}}^{p-1} \cdot\|K f(s)\|_{L^{p}} \mathrm{~d} s \leq C \int_{0}^{t}\|f(s)\|_{L^{p}}^{p} \mathrm{~d} s .
$$

As for the last term on the right-hand side of (5.4), it holds from (2.17) that

$$
\begin{aligned}
\left|\left\langle|f|^{p-2} f,-L_{\sqrt{\mu} f_{*}} f+\Gamma(f, f)\right\rangle\right| & \leq\left\|\nu^{1 / p} f\right\|_{L^{p}}^{p-1}\left\|\nu^{-1 / p^{\prime}}\left|-L_{\sqrt{\mu} f_{*}} f+\Gamma(f, f)\right|\right\|_{L^{p}} \\
& \leq C\left\{\left\|w f_{*}\right\|_{L^{\infty}}+\sup _{0 \leq s \leq t}\|w f(s)\|_{L^{\infty}}\right\} \cdot\left\|\nu^{1 / p} f\right\|_{L^{p}}^{p} \\
& \leq C(\bar{M}+1)\left\|\nu^{1 / p} f\right\|_{L^{p}}^{p} .
\end{aligned}
$$


To treat the boundary term $|f|_{L^{p}\left(\gamma_{-}\right)}$, the same as before, we introduce the cutoff function $\mathbf{1}_{\gamma_{+}^{\varepsilon}}$ near the grazing set $\gamma_{+}^{\varepsilon}$ defined in (4.7). Then by a direct computation, we have

$$
\begin{aligned}
|f|_{L^{p}\left(\gamma_{-}\right)}^{p} & \leq C\left|P_{\gamma} f\right|_{L^{p}\left(\gamma_{-}\right)}^{p}+C|r|_{L^{p}\left(\gamma_{-}\right)}^{p} \leq C\left|P_{\gamma} f\right|_{L^{p}\left(\gamma_{-}\right)}^{p}+C \delta^{p}|f|_{L^{p}\left(\gamma_{+}\right)}^{p} \\
& \leq \int_{\gamma_{-}} \mu^{p / 2}(v)|n(x) \cdot v| \mathrm{d} \gamma\left(\int_{\mathcal{V}(x)} \sqrt{\mu(u)}\left\{f \mathbf{1}_{\gamma_{+}^{\varepsilon}}+f \mathbf{1}_{\gamma_{+} \backslash \gamma_{+}^{\varepsilon}}\right\}|n(x) \cdot u| \mathrm{d} u\right)^{p}+C \delta^{p}|f|_{L^{p}\left(\gamma_{+}\right)}^{p} \\
& \leq C\left\{\varepsilon^{p}+C \delta^{p}\right\} \cdot|f|_{L^{p}\left(\gamma_{+}\right)}^{p}+C\left|f \mathbf{1}_{\gamma_{+} \backslash \gamma_{+}^{\varepsilon}}\right|_{L^{p}\left(\gamma_{+}\right)}^{p} .
\end{aligned}
$$

From the trace estimate (4.9), it holds that

$$
\begin{aligned}
\int_{0}^{t}\left|f \mathbf{1}_{\gamma_{+} \backslash \gamma_{+}^{\varepsilon}}(s)\right|_{L^{p}\left(\gamma_{+}\right)}^{p} \mathrm{~d} s & =\left.\left.\int_{0}^{t}|| f\right|^{p} \mathbf{1}_{\gamma_{+} \backslash \gamma_{+}^{\varepsilon}}(s)\right|_{L^{1}\left(\gamma_{+}\right)} \mathrm{d} s \\
\leq C_{\varepsilon, \Omega} & \left\{\left\|f_{0}\right\|_{L^{p}}^{p}+\int_{0}^{t}\|f(s)\|_{L^{p}}^{p} \mathrm{~d} s+\int_{0}^{t}\left\|\left[\partial_{t}+v \cdot \nabla_{x}\right]|f(s)|^{p}\right\|_{L^{1}} \mathrm{~d} s\right\} .
\end{aligned}
$$

Notice that

$$
\left[\partial_{t}+v \cdot \nabla_{x}\right]|f|^{p}=p|f|^{p-2} f\left\{\partial_{t} f+v \cdot \nabla_{x} f\right\}=p|f|^{p-2} f\left\{-\nu f+K f+\Gamma(f, f)-L_{\sqrt{\mu} f_{*}} f\right\} .
$$

Then from (5.5) and (5.6), it holds that

$$
\int_{0}^{t}\left\|\left[\partial_{t}+v \cdot \nabla_{x}\right]|f(s)|^{p}\right\|_{L^{1}} \mathrm{~d} s \leq C(\bar{M}+1) \int_{0}^{t}\|f(s)\|_{L^{p}}^{p} \mathrm{~d} s .
$$

Combining this with (5.7) and (5.8), we obtain that

$$
\int_{0}^{t}|f(s)|_{L^{p}\left(\gamma_{-}\right)}^{p} \mathrm{~d} s \leq C\left\{\varepsilon^{p}+\delta^{p}\right\} \cdot \int_{0}^{t}|f(s)|_{L^{p}\left(\gamma_{+}\right)}^{p} \mathrm{~d} s+C_{\varepsilon}\left\|f_{0}\right\|_{L^{p}}^{p}+C_{\varepsilon}(\bar{M}+1) \int_{0}^{t}\|f(s)\|_{L^{p}}^{p} \mathrm{~d} s .
$$

Substituting (5.5), (5.6) and (5.9) into (5.4), and then taking both $\varepsilon>0$ as well as $\delta>0$ suitably small, we have

$$
\|f(t)\|_{L^{p}}^{p}+\int_{0}^{t}|f(s)|_{L^{p}}^{p} \mathrm{~d} s+\int_{0}^{t}\left\|\nu^{1 / p} f(s)\right\|_{L^{p}}^{p} \mathrm{~d} s \leq C\left\|f_{0}\right\|_{L^{p}}^{p}+C(\bar{M}+1) \int_{0}^{t}\|f(s)\|_{L^{p}}^{p} \mathrm{~d} s .
$$

Then (5.3) follows from Gronwall's inequality. Therefore, the proof of Lemma 5.2 is complete.

\section{2. $L_{x, v}^{\infty}$-estimate.}

Lemma 5.3. Under the a priori assumption (5.1), for $t \in\left[0, \min \left\{T, T_{0}\right\}\right]$ and for almost every $(x, v) \in \bar{\Omega} \times \mathbb{R}^{3} \backslash \gamma_{0}$, it holds that:

$$
|h(t, x, v)| \leq S(t)+\int_{\max \left\{t_{1}, 0\right\}}^{t} I(t, s)|w \Gamma(f, f)(s, x-(t-s) v, v)| \mathrm{d} s,
$$

where

$$
\begin{aligned}
S(t)= & C e^{-\lambda t^{\alpha}} M_{0}+C T_{0}^{5 / 2}\left\{m^{3+\kappa}+\delta+2^{-\hat{C}_{4} T_{0}^{5 / 4}}+\frac{1}{N}+\frac{1}{N^{\beta-4}}\right\} \cdot\left\{\bar{M}+\bar{M}^{2}\right\} \\
& +C_{N, T_{0}, m}\left\{\sup _{0 \leq s \leq t}\|f(s)\|_{L^{p}}+\sup _{0 \leq s \leq t}\|f(s)\|_{L^{p}}^{2}\right\} .
\end{aligned}
$$

Here the positive constants $T_{0}$ and $N$ can be taken arbitrarily large and $m$ can be taken arbitrarily small.

Proof. We denote $\tilde{G}(t)$ as the solution operator of (4.11) provided by Proposition 4.4 Then the solution $h$ of (4.3) is given in terms of Duhamel's formula as

$$
h(t, x, v)=\left(\tilde{G}(t) h_{0}\right)(t, x, v)+\int_{0}^{t}\left(\tilde{G}(t-s)\left[-w L_{\sqrt{\mu} f_{*}} f(s)+w \Gamma(f, f)(s)\right] \mathrm{d} s\right)(t, x, v) .
$$


Using (4.14) and (2.16), we have

$$
\left\|\tilde{G}(t) h_{0}\right\|_{L^{\infty}} \leq C e^{-\lambda_{0} t^{\alpha}}\left\|h_{0}\right\|_{L^{\infty}}
$$

and

$$
\left|\int_{0}^{t} \tilde{G}(t-s) w L_{\sqrt{\mu} f_{*}} f(s) \mathrm{d} s\right| \leq C\left\|w f_{*}\right\|_{L^{\infty}} \cdot \int_{0}^{t} e^{-\lambda_{0}(t-s)^{\alpha}}\|h(s)\|_{L^{\infty}} \mathrm{d} s \leq C \delta \sup _{0 \leq s \leq t}\|h(s)\|_{L^{\infty}} .
$$

To estimate the last term on the right-hand side of (5.11), denoting

$$
Z(t, x, v):=(\tilde{G}(t-s) w \Gamma(f, f)(s))(t, x, v)
$$

and then applying the mild formulation (4.12) to $Z(t, x, v)$, we obtain that

$$
(\tilde{G}(t-s) w \Gamma(f, f))(t, x, v)=\sum_{i=1}^{3} H_{i}+\mathbf{1}_{\left\{t_{1}>s\right\}} \sum_{i=4}^{11} H_{i}
$$

where

$$
\begin{aligned}
& H_{1}=\mathbf{1}_{\left\{t_{1} \leq s\right\}} I(t, s) w \Gamma(f, f)(s, x-(t-s) v, v) \\
& H_{2}+H_{3}=\int_{\max \left\{t_{1}, s\right\}}^{t} I(t, \tau)\left[K_{w}^{m}+K_{w}^{c}\right] Z(\tau, x-(t-\tau) v, v) \mathrm{d} \tau, \quad H_{4}=I\left(t, t_{1}\right) w r[Z]\left(t_{1}, x_{1}, v\right) \\
& H_{5}=\frac{I\left(t, t_{1}\right)}{\tilde{w}(v)} \int_{\prod_{j=1}^{k-1} \mathcal{V}_{j}} \sum_{l=1}^{k-1} \mathbf{1}_{\left\{t_{l+1} \leq s<t_{l}\right\}} w \Gamma(f, f)\left(s, x_{l}-\left(t_{l}-s\right) v_{l}, v_{l}\right) \mathrm{d} \Sigma_{l}(s) \\
& H_{6}+H_{7}=\frac{I\left(t, t_{1}\right)}{\tilde{w}(v)} \int_{\prod_{j=1}^{k-1} \mathcal{V}_{j}} \int_{s}^{t_{l}} \sum_{l=1}^{k-1} \mathbf{1}_{\left\{t_{l+1} \leq s<t_{l}\right\}}\left[K_{w}^{m}+K_{w}^{c}\right] Z\left(\tau, x_{l}-\left(t_{l}-\tau\right) v_{l}, v_{l}\right) \mathrm{d} \Sigma_{l}(\tau) \mathrm{d} \tau \\
& H_{8}+H_{9}=\frac{I\left(t, t_{1}\right)}{\tilde{w}(v)} \int_{\prod_{j=1}^{k-1} \mathcal{V}_{j}} \int_{t_{l+1}}^{t_{l}} \sum_{l=1}^{k-1} \mathbf{1}_{\left\{t_{l+1}>s\right\}}\left[K_{w}^{m}+K_{w}^{c}\right] Z\left(\tau, x_{l}-\left(t_{l}-\tau\right) v_{l}, v_{l}\right) \mathrm{d} \Sigma_{l}(\tau) \mathrm{d} \tau \\
& H_{10}=\frac{I\left(t, t_{1}\right)}{\tilde{w}(v)} \int_{\prod_{j=1}^{k-1} \mathcal{V}_{j}} \sum_{l=1}^{k-2} \mathbf{1}_{\left\{t_{l+1}>s\right\}} w r[Z]\left(t_{l+1}, x_{l+1}, v_{l}\right) \mathrm{d} \Sigma_{l}\left(t_{l+1}\right) \\
& H_{11}=\frac{I\left(t, t_{1}\right)}{\tilde{w}(v)} \int_{\prod_{j=1}^{k-1} \mathcal{V}_{j}} \mathbf{1}_{\left\{t_{k}>s\right\}} Z\left(t_{k}, x_{k}, v_{k-1}\right) \mathrm{d} \Sigma_{k-1}\left(t_{k}\right),
\end{aligned}
$$

and $r$ is defined in (4.5). Here the same as before, $k=\hat{C}_{3} T_{0}^{5 / 4}$ such that (4.13) holds for $\eta=\frac{5}{16}$. We first consider terms $H_{2}, H_{5}$ and $H_{7}$ involving $K_{w}^{m}$. On one hand, similar for obtaining (4.20), we have

$$
\begin{aligned}
\left|H_{2}\right| & \leq \int_{\max \left\{t_{1}, s\right\}}^{t} I(t, \tau)\left|K_{w}^{m} Z(\tau, x-(t-\tau) v, v)\right| \mathrm{d} \tau \\
& \leq \int_{\max \left\{t_{1}, s\right\}}^{t} I(t, \tau)\left\{\mathbf{1}_{\left\{|v| \leq d_{\Omega}\right\}}+\mathbf{1}_{\left\{|v|>d_{\Omega}\right\}}\right\}\left|K_{w}^{m} Z(\tau, x-(t-\tau) v, v)\right| \mathrm{d} \tau \\
& \leq C m^{3+\kappa} \int_{\max \left\{t_{1}, s\right\}}^{t} e^{-\bar{\nu}_{0}(t-\tau)}\|\tilde{G}(\tau-s) w \Gamma(f, f)(s)\|_{L^{\infty}} \mathrm{d} \tau \\
& \leq C m^{3+\kappa}\|h(s)\|_{L^{\infty}}^{2} \cdot \int_{\max \left\{t_{1}, s\right\}}^{t} e^{-\bar{\nu}_{0}(t-\tau)-\lambda_{0}(\tau-s)^{\alpha}} \mathrm{d} \tau \\
& \leq C m^{3+\kappa} e^{-\lambda_{0}(t-s)^{\alpha}}\|h(s)\|_{L^{\infty}}^{2} .
\end{aligned}
$$


On the other hand, similar for obtaining (4.23), we have

$$
\begin{aligned}
&\left|H_{6}\right| \leq \sum_{l=1}^{k-1} \sum_{m=1}^{l} C\left\{\int_{\prod_{j=1}^{l} \mathcal{V}_{j}} \mathbf{1}_{\left\{t_{l+1} \leq s<t_{l}\right\}} \times \mathbf{1}_{\left\{\left|v_{m}\right|=\max \left[\left|v_{1}\right|,\left|v_{2}\right|, \ldots\left|v_{l}\right|\right]\right\}} e^{\frac{5\left|v_{m}\right|^{2}}{16}} \prod_{j=1}^{l} \mathrm{~d} \sigma_{j}\right. \\
&\left.\times \int_{s}^{t_{l}} e^{-\lambda_{1}(t-\tau)^{\alpha}}\left\|K_{w}^{m} Z(\tau)\right\|_{L^{\infty}} \mathrm{d} \tau\right\} \\
& \leq C k^{2} m^{3+\kappa} e^{-\lambda_{0}(t-s)^{\alpha}}\|h(s)\|_{L^{\infty}}^{2} \cdot \sup _{j}\left|\int_{\mathcal{V}_{j}} e^{\frac{5\left|v_{j}\right|^{2}}{16}} \mathrm{~d} \sigma_{j}\right| \\
& \leq C k^{2} m^{3+\kappa} e^{-\lambda_{0}(t-s)^{\alpha}}\|h(s)\|_{L^{\infty}}^{2} .
\end{aligned}
$$

Similarly, it holds that

$$
\left|H_{8}\right| \leq C k^{2} m^{3+\kappa} e^{-\lambda_{0}(t-s)^{\alpha}}\|h(s)\|_{L^{\infty}}^{2} .
$$

For the terms $H_{4}$ and $H_{10}$ involving $r$, we see from (4.14) and (4.5) that

$$
|r[Z](\tau)|_{L^{\infty}\left(\gamma_{-}\right)} \leq C \delta e^{-\lambda_{0}(\tau-s)^{\alpha}}|h(s)|_{L^{\infty}\left(\gamma_{+}\right)}^{2} .
$$

Therefore, similar for obtaining (5.16), we have

$$
\begin{aligned}
\left|H_{10}\right| \leq & \sum_{l=1}^{k-1} \sum_{m=1}^{l} C\left\{\int_{\prod_{j=1}^{l} \mathcal{V}_{j}} \mathbf{1}_{\left\{t_{l+1}>s\right\}}\right. \\
& \left.\times \mathbf{1}_{\left\{\left|v_{m}\right|=\max \left[\left|v_{1}\right|,\left|v_{2}\right|, \ldots\left|v_{l}\right|\right]\right\}} e^{\frac{5\left|v_{m}\right|^{2}}{16}} e^{-\lambda_{1}\left(t-t_{l+1}\right)^{\alpha}}\left|r[Z]\left(t_{l+1}\right)\right|_{L^{\infty}\left(\gamma_{-}\right)} \prod_{j=1}^{l} \mathrm{~d} \sigma_{j}\right\} \\
\leq & C k^{2} \delta e^{-\lambda_{0}(t-s)^{\alpha}}|h(s)|_{L^{\infty}\left(\gamma_{+}\right)}^{2} \cdot \sup _{j}\left|\int_{\mathcal{V}_{j}} e^{\frac{5\left|v_{j}\right|^{2}}{16}} \mathrm{~d} \sigma_{j}\right| \\
\leq & C k^{2} \delta e^{-\lambda_{0}(t-s)^{\alpha}}|h(s)|_{L^{\infty}\left(\gamma_{+}\right)}^{2}
\end{aligned}
$$

and

$$
\begin{aligned}
\left|H_{4}\right| & \leq C \mathbf{1}_{\left\{t_{1}>s\right\}} I\left(t, t_{1}\right)\left\{\mathbf{1}_{\left\{|v| \leq d_{\Omega}\right\}}+\mathbf{1}_{\left\{|v|>d_{\Omega}\right\}}\right\}\left|w r[Z]\left(t_{1}, x_{1}, v\right)\right| \\
& \leq C e^{-\bar{\nu}_{0}\left(t-t_{1}\right)}\left|w r[Z]\left(t_{1}, x_{1}, v\right)\right| \leq C \delta e^{-\lambda_{0}(t-s)^{\alpha}}|h(s)|_{L^{\infty}\left(\gamma_{+}\right)}^{2} .
\end{aligned}
$$

For $H_{11}$, we note that

$$
\left|Z\left(t_{k}, x_{k}, v_{k-1}\right)\right| \leq\left|\tilde{G}\left(t_{k}-s\right) w \Gamma(f, f)(s)\right|_{L^{\infty}\left(\gamma_{-}\right)} \leq e^{-\lambda_{0}\left(t_{k}-s\right)^{\alpha}}\|h(s)\|_{L^{\infty}}^{2} .
$$

Then by (4.13), we have

$$
\left|H_{11}\right| \leq C e^{-\lambda_{0}(t-s)^{\alpha}}\|h(s)\|_{L^{\infty}}^{2} \cdot\left(\frac{1}{2}\right)^{\hat{C}_{4} T_{0}^{5 / 4}} .
$$

For the terms $H_{7}$ and $H_{9}$ involving $K_{w}^{c}$, similar for obtaining (4.28), we have

$$
\begin{aligned}
\left|H_{8}\right| \leq C & \sum_{l=1}^{k-1} \sum_{m=1}^{l} \int_{\Pi_{j=1}^{l-1} \mathcal{V}_{j}} \mathrm{~d} \sigma_{l-1} \cdots \mathrm{d} \sigma_{1} \\
& \times \int_{\mathcal{V}_{l}} \int_{\mathbb{R}^{3}} \int_{s}^{t_{l}} e^{-\lambda_{1}(t-\tau)^{\alpha}} \mathbf{1}_{\left\{t_{l+1} \leq s<t_{l}\right\}} e^{\frac{5\left|v_{m}\right|^{2}}{16}}\left|k_{w}^{c}\left(v_{l}, v^{\prime}\right) Z\left(\tau, x_{l}-v_{l}\left(t_{l}-\tau\right), v^{\prime}\right)\right| \mathrm{d} \tau \mathrm{d} v^{\prime} \mathrm{d} \sigma_{l}
\end{aligned}
$$


and further split it as

$$
\begin{aligned}
\left|H_{8}\right| \leq & C \sum_{l=1}^{k-1} \sum_{m=1}^{l-1} \int_{\Pi_{j=1}^{l-1} \mathcal{V}_{j}} e^{\frac{5\left|v_{m}\right|^{2}}{16}} \mathrm{~d} \sigma_{l-1} \cdots \mathrm{d} \sigma_{1} \int_{\mathcal{V}_{l} \cap\left\{\left|v_{l}\right| \geq N\right\}} \int_{\mathbb{R}^{3}} \int_{s}^{t_{l}} \bar{\Delta} \mathrm{d} \tau \mathrm{d} v^{\prime} \mathrm{d} \sigma_{l} \\
& +C \sum_{l=1}^{k-1} \sum_{m=1}^{l-1} \int_{\Pi_{j=1}^{l-1} \mathcal{V}_{j}} e^{\frac{5\left|v_{m}\right|^{2}}{16}} \mathrm{~d} \sigma_{l-1} \cdots \mathrm{d} \sigma_{1} \int_{\mathcal{V}_{l} \cap\left\{\left|v_{l}\right| \leq N\right\}} \int_{\mathbb{R}^{3}} \int_{t_{l}-\frac{1}{N}}^{t_{l}} \bar{\Delta} \mathrm{d} \tau \mathrm{d} v^{\prime} \mathrm{d} \sigma_{l} \\
& +C \sum_{l=1}^{k-1} \sum_{m=1}^{l-1} \int_{\Pi_{j=1}^{l-1} \mathcal{V}_{j}} e^{\frac{5\left|v_{m}\right|^{2}}{16}} \mathrm{~d} \sigma_{l-1} \cdots \mathrm{d} \sigma_{1} \int_{\mathcal{V}_{l} \cap\left\{\left|v_{l}\right| \leq N\right\}} \int_{\left\{\left|v^{\prime}\right| \geq 2 N\right\}} \int_{s}^{t_{l}-\frac{1}{N}} \bar{\Delta} \mathrm{d} \tau \mathrm{d} v^{\prime} \mathrm{d} \sigma_{l} \\
& +C \sum_{l=1}^{k-1} \sum_{m=1}^{l-1} \int_{\Pi_{j=1}^{l-1} \mathcal{V}_{j}} e^{\frac{5\left|v_{m}\right|^{2}}{16}} \mathrm{~d} \sigma_{l-1} \cdots \mathrm{d} \sigma_{1} \int_{\mathcal{V}_{l} \cap\left\{\left|v_{l}\right| \leq N\right\}} \int_{\left\{\left|v^{\prime}\right| \leq 2 N\right\}} \int_{s}^{t_{l}-\frac{1}{N}} \bar{\Delta} \mathrm{d} \tau \mathrm{d} v^{\prime} \mathrm{d} \sigma_{l},
\end{aligned}
$$

where

$$
\bar{\Delta}:=e^{-\lambda_{1}(t-\tau)^{\alpha}} \mathbf{1}_{\left\{t_{l+1} \leq s<t_{l}\right\}} e^{\frac{5\left|v_{l}\right|^{2}}{16}}\left|k_{w}^{c}\left(v_{l}, v^{\prime}\right) Z\left(\tau, x_{l}-v_{l}\left(t_{l}-\tau\right), v^{\prime}\right)\right| .
$$

Then it follows that

$$
\begin{aligned}
\left|H_{8}\right| \leq & \frac{C k^{2} e^{-\lambda_{0}(t-s)^{\alpha}}}{N} \sup _{s \leq \tau \leq t}\|h(\tau)\|_{L^{\infty}}^{2} \\
& +C \sum_{l=1}^{k-1} \sum_{m=1}^{l-1} \int_{\Pi_{j=1}^{l-1} \mathcal{V}_{j}} e^{\frac{5\left|v_{m}\right|^{2}}{16}} \mathrm{~d} \sigma_{l-1} \cdots \mathrm{d} \sigma_{1} \int_{\mathcal{V}_{l} \cap\left\{\left|v_{l}\right| \leq N\right\}} \int_{\left\{\left|v^{\prime}\right| \leq 2 N\right\}} \int_{s}^{t_{l}-\frac{1}{N}} \bar{\Delta} \mathrm{d} \tau \mathrm{d} v^{\prime} \mathrm{d} \sigma_{l},
\end{aligned}
$$

By Hölder inequality, it holds that

$$
\begin{aligned}
& \int_{\mathcal{V}_{l} \cap\left\{\left|v_{l}\right| \leq N\right\}} \int_{\left\{\left|v^{\prime}\right| \leq 2 N\right\}} \int_{s}^{t_{l}-\frac{1}{N}} \bar{\Delta} \mathrm{d} \tau \mathrm{d} v^{\prime} \mathrm{d} \sigma_{l} \\
& \leq C \int_{s}^{t_{l}-\frac{1}{N}} e^{-\lambda_{1}(t-\tau)^{\alpha}} \mathrm{d} \tau\left(\int_{\mathcal{V}_{l} \cap\left\{\left|v_{l}\right| \leq N\right\}} \int_{\left\{\left|v^{\prime}\right| \leq 2 N\right\}} e^{-\frac{\left|v_{l}\right|^{2}}{8}}\left|k_{w}^{c}\left(v_{l}, v^{\prime}\right)\right|^{p^{\prime}} \mathrm{d} v^{\prime} \mathrm{d} v_{l}\right)^{\frac{1}{p^{\prime}}} \\
& \quad \times\left(\int_{\mathcal{V}_{l} \cap\left\{\left|v_{l}\right| \leq N\right\}} \int_{\left\{\left|v^{\prime}\right| \leq 2 N\right\}} 1_{\left\{t_{l+1} \leq s<t_{l}\right\}}\left|Z\left(\tau, x_{l}-\left(t_{l}-\tau\right) v_{l}, v^{\prime}\right)\right|^{p} \mathrm{~d} v^{\prime} \mathrm{d} v_{l}\right)^{\frac{1}{p}} .
\end{aligned}
$$

Since $1<p^{\prime}=\frac{p}{p-1}<3$, then by (2.12) with $a=1$, it holds that

$$
\int_{\mathcal{V}_{l} \cap\left\{\left|v_{l}\right| \leq N\right\}} \int_{\left\{\left|v^{\prime}\right| \leq 2 N\right\}} e^{-\frac{\left|v_{l}\right|^{2}}{8}}\left|k_{w}^{c}\left(v_{l}, v^{\prime}\right)\right|^{p^{\prime}} \mathrm{d} v^{\prime} \mathrm{d} v_{l} \leq C m^{p^{\prime}(\kappa-1)} .
$$

Therefore, it holds that

$$
\begin{aligned}
\text { R.H.S. of (5.22) } \leq & C_{N} m^{\kappa-1} \int_{s}^{t_{l}-\frac{1}{N}} e^{-\lambda_{1}(t-\tau)^{\alpha}} \mathrm{d} \tau \\
& \times\left(\int_{\mathcal{V}_{l} \cap\left\{\left|v_{l}\right| \leq N\right\}} \int_{\left\{\left|v^{\prime}\right| \leq 2 N\right\}} \mathbf{1}_{\left\{t_{l+1} \leq s<t_{l}\right\}}\left|\frac{Z}{w}\left(\tau, x_{l}-\left(t_{l}-\tau\right) v_{l}, v^{\prime}\right)\right|^{p} \mathrm{~d} v^{\prime} \mathrm{d} v_{l}\right)^{\frac{1}{p}} .
\end{aligned}
$$

Note that $y_{l}:=x_{l}-\left(t_{l}-\tau\right) v_{l} \in \Omega$ for $s \leq \tau \leq t_{l}-\frac{1}{N}$. Then making chang of variable $v_{l} \rightarrow y_{l}$, we obtain that

$$
\int_{\mathcal{V}_{l} \cap\left\{\left|v_{l}\right| \leq N\right\}} \int_{\left\{\left|v^{\prime}\right| \leq 2 N\right\}} \int_{s}^{t_{l}-\frac{1}{N}} \bar{\Delta} \mathrm{d} \tau \mathrm{d} v^{\prime} \mathrm{d} \sigma_{l} \leq C_{N, m} \int_{s}^{t_{l}-\frac{1}{N}} e^{-\lambda_{1}(t-\tau)^{\alpha}}\left\|\frac{Z(\tau)}{w}\right\|_{L^{p}} \mathrm{~d} \tau .
$$


Similar for obtaining (5.3), we have

$$
\begin{aligned}
\left\|\frac{Z(\tau)}{w}\right\|_{L^{p}} & =\left\|\frac{\tilde{G}(\tau-s) w \Gamma(f, f)(s)}{w}\right\|_{L^{p}} \leq C e^{C(\tau-s)}\|\Gamma(f, f)(s)\|_{L^{p}} \\
& \leq C_{T_{0}} e^{-\lambda_{0}(\tau-s)^{\alpha}}\|\Gamma(f, f)(s)\|_{L^{p}} \leq C_{T_{0}} e^{-\lambda_{0}(\tau-s)^{\alpha}}\|h(s)\|_{L^{\infty}} \cdot\|f(s)\|_{L^{p}} \\
& \leq \frac{C e^{-\lambda_{0}(\tau-s)^{\alpha}}}{N}\|h(s)\|_{L^{\infty}}^{2}+C_{N, T_{0}} e^{-\lambda_{0}(\tau-s)^{\alpha}}\|f(s)\|_{L^{p}}^{2},
\end{aligned}
$$

which implies that

$$
\int_{\mathcal{V}_{l} \cap\left\{\left|v_{l}\right| \leq N\right\}} \int_{\left\{\left|v^{\prime}\right| \leq 2 N\right\}} \int_{s}^{t_{l}-\frac{1}{N}} \bar{\Delta} \mathrm{d} \tau \mathrm{d} v^{\prime} \mathrm{d} \sigma_{l} \leq \frac{C e^{-\lambda_{0}(t-s)^{\alpha}}}{N}\|h(s)\|_{L^{\infty}}^{2}+C_{N, T_{0}, m} e^{-\lambda_{0}(t-s)^{\alpha}}\|f(s)\|_{L^{p}}^{2} .
$$

Substituting this into (5.21), we have

$$
\left|H_{8}\right| \leq \frac{C k^{2} e^{-\lambda_{0}(t-s)^{\alpha}}}{N} \sup _{0 \leq s \leq t}\|h(s)\|_{L^{\infty}}^{2}+C_{N, T_{0}, m} e^{-\lambda_{0}(t-s)^{\alpha}} \sup _{0 \leq s \leq t}\|f(s)\|_{L^{p}}^{2}
$$

Similarly, we have

$$
\left|H_{10}\right| \leq \frac{C k^{2} e^{-\lambda_{0}(t-s)^{\alpha}}}{N} \sup _{0 \leq s \leq t}\|h(s)\|_{L^{\infty}}^{2}+C_{N, T_{0}, m} e^{-\lambda_{0}(t-s)^{\alpha}} \sup _{0 \leq s \leq t}\|f(s)\|_{L^{p}}^{2} .
$$

For $H_{5}$, similar as (4.23), we have

$$
\begin{aligned}
\int_{0}^{t}\left|H_{5}\right| \mathrm{d} s \leq & C \int_{0}^{t} e^{-\lambda_{1}(t-s)^{\alpha}} \mathrm{d} s \sum_{l=1}^{k-1} \sum_{m=1}^{l} \int_{\Pi_{j=1}^{l-1} \mathcal{V}_{j}} \mathrm{~d} \sigma_{l-1} \cdots \mathrm{d} \sigma_{1} \\
& \times \int_{\mathcal{V}_{l}} \int_{\mathbb{R}^{3}} \mathbf{1}_{\left\{t_{l+1} \leq s<t_{l}\right\}} e^{\frac{5\left|v_{m}\right|^{2}}{16}}\left|w \Gamma(f, f)\left(s, x_{l}-\left(t_{l}-s\right) v_{l}, v_{l}\right)\right| \mathrm{d} \tau \mathrm{d} v^{\prime} \mathrm{d} \sigma_{l} .
\end{aligned}
$$

Then it follows that

$$
\begin{aligned}
\int_{0}^{t}\left|H_{5}\right| \mathrm{d} s \leq & C \int_{0}^{t} e^{-\lambda_{1}(t-s)^{\alpha}}\|h(s)\|_{L^{\infty}} \mathrm{d} s \sum_{l=1}^{k-1} \sum_{m=1}^{l-1} \int_{\Pi_{j=1}^{l-1} \mathcal{V}_{j}} e^{\frac{5\left|v_{m}\right|^{2}}{16}} \mathrm{~d} \sigma_{l-1} \cdots \mathrm{d} \sigma_{1} \\
& \times \int_{\mathcal{V}_{l}} e^{-\frac{\left|v_{l}\right|^{2}}{8}} 1_{\left\{t_{l+1} \leq s<t_{l}\right\}} \mathrm{d} v_{l}\left\{\int_{\mathbb{R}^{3}}\left|h\left(s, x_{l}-\left(t_{l}-s\right) v_{l}, v^{\prime}\right)\right|^{p}\left\langle v^{\prime}\right\rangle^{-4-p(\beta-4)} \mathrm{d} v^{\prime}\right\}^{1 / p} \\
\leq & C \int_{0}^{t} e^{-\lambda_{1}(t-s)^{\alpha}}\|h(s)\|_{L^{\infty}} \mathrm{d} s \\
& \times\left(\sum_{l=1}^{k-1} \sum_{m=1}^{l-1} \int_{\Pi_{j=1}^{l-1} \mathcal{V}_{j}} e^{\frac{5\left|v_{m}\right|^{2}}{16}} \mathrm{~d} \sigma_{l-1} \cdots \mathrm{d} \sigma_{1}\left\{\int_{\mathcal{V}_{l}} \int_{\mathbb{R}^{3}} \tilde{\Delta} \mathrm{d} v_{l} \mathrm{~d} v^{\prime}\right\}^{1 / p}\right)
\end{aligned}
$$

where we have used (2.20) and (2.21) in the first inequality, and also denoted that

$$
\tilde{\Delta}:=e^{-\frac{\left|v_{l}\right|^{2}}{8}} \mathbf{1}_{\left\{t_{l+1} \leq s<t_{l}\right\}}\left|h\left(s, x_{l}-\left(t_{l}-s\right) v_{l}, v^{\prime}\right)\right|^{p}\left\langle v^{\prime}\right\rangle^{-4-p(\beta-4)} .
$$

Now, we consider the integral in (5.26) over either $\left\{\left|v_{l}\right| \geq N\right\}$ or $\left\{\left|v_{l}\right| \leq N,\left|v^{\prime}\right| \geq N\right\}$ or $\left\{\left|v_{l}\right| \leq\right.$ $\left.N,\left|v^{\prime}\right| \leq N, t_{l}-1 / N \leq s \leq t_{l}\right\}$ or $\left\{\left|v_{l}\right| \leq N,\left|v^{\prime}\right| \leq N, 0 \leq s \leq t_{l}-1 / N\right\}$. Over $\left\{\left|v_{l}\right| \geq N\right\}$ or $\left\{\left|v_{l}\right| \leq N,\left|v^{\prime}\right| \geq N\right\}$ or $\left\{\left|v_{l}\right| \leq N,\left|v^{\prime}\right| \leq N, t_{l}-1 / N \leq s \leq t_{l}\right\}$, it is bounded by

$$
C k^{2}\left(\frac{1}{N}+\frac{1}{N^{\beta-4}}\right) \bar{M}^{2} \text {. }
$$


Over $\left\{\left|v_{l}\right| \leq N,\left|v^{\prime}\right| \leq N, 0 \leq s \leq t_{l}-1 / N\right\}$, it is bounded by

$$
\begin{aligned}
& C_{N} \sum_{l=1}^{k-1} \sum_{m=1}^{l-1} \int_{0}^{t} e^{-\lambda_{1}(t-s)^{\alpha}}\|h(s)\|_{L^{\infty}} \mathrm{d} s \int_{\Pi_{j=1}^{l-1} \mathcal{V}_{j}} e^{\frac{5\left|v_{m}\right|^{2}}{16}} \mathrm{~d} \sigma_{l-1} \cdots \mathrm{d} \sigma_{1} \\
& \quad \times\left\{\int_{\mathcal{V}_{l} \cap\left\{\left|v_{l}\right| \leq N\right\}} \int_{\left\{\left|v^{\prime}\right| \leq N\right\}} e^{-\frac{\left|v_{l}\right|^{2}}{8}} 1_{\left\{t_{l+1} \leq s<t_{l}-\frac{1}{N}\right\}}\left|f\left(s, x_{l}-\left(t_{l}-s\right) v_{l}, v^{\prime}\right)\right|^{p} \mathrm{~d} v_{l} \mathrm{~d} v^{\prime}\right\}^{1 / p} \\
& \leq C_{N} k^{2} \int_{0}^{t} e^{-\lambda_{1}(t-s)^{\alpha}}\|h(s)\|_{L^{\infty}} \cdot\|f(s)\|_{L^{p}} \mathrm{~d} s \leq \frac{C k^{2}}{N} \bar{M}^{2}+C_{N} k^{2} \sup _{0 \leq s \leq t}\|f(s)\|_{L^{p}}^{2},
\end{aligned}
$$

where we have used the change of variable $v_{l} \rightarrow y_{l}:=x_{l}-\left(t_{l}-s\right) v_{l}$ above. Therefore, for $H_{5}$, it holds that

$$
\int_{0}^{t}\left|H_{5}\right| \mathrm{d} s \leq C k^{2}\left(\frac{1}{N}+\frac{1}{N^{\beta-4}}\right) \bar{M}^{2}+C_{N} k^{2} \sup _{0 \leq s \leq t}\|f(s)\|_{L^{p}}^{2}
$$

Substituting (5.15), (5.16), (5.17), (5.18), (5.19), (5.20), (5.24) (5.25) and (5.27) into (5.14), we have

$$
\begin{aligned}
& \int_{0}^{t}|(\tilde{G}(t-s) w \Gamma(f, f)(s))(t, x, v)| \mathrm{d} s \\
& \leq \int_{0}^{t} \mathbf{1}_{\left\{t_{1} \leq s\right\}} I(t, s) w \Gamma(f, f)(s, x-(t-s) v, v) \mathrm{d} s \\
& \quad+\int_{0}^{t} \mathrm{~d} s \int_{\max \left\{t_{1}, s\right\}}^{t} I(t, \tau) \mathrm{d} \tau \int_{\mathbb{R}^{3}}\left|k_{w}^{c}(v, u)(\tilde{G}(\tau-s) w \Gamma(f, f)(s))(\tau, x-(t-\tau) v, u)\right| \mathrm{d} u \\
& \quad+C T_{0}^{5 / 2} \bar{M}^{2}\left\{m^{3+\kappa}+\delta+2^{-\hat{C}_{4} T_{0}^{5 / 4}}+\frac{1}{N}+\frac{1}{N^{\beta-4}}\right\}+C_{N, T_{0}, m} \sup _{0 \leq s \leq t}\|f(s)\|_{L^{p}}^{2} \\
& :=H_{12}+H_{13}+H_{14} .
\end{aligned}
$$

To further estimate $H_{13}$, we denote $x^{\prime}=x-(t-\tau) v$ and $\tau_{1}^{\prime}=t_{1}\left(\tau, x^{\prime}, u\right)$. Then by Fubini Theorem and (5.28), it holds that

$$
\begin{aligned}
\left|H_{13}\right| & =\left|\int_{\max \left\{t_{1}, 0\right\}}^{t} \int_{\mathbb{R}^{3}} I(t, \tau) k_{w}^{c}(v, u) \mathrm{d} u \mathrm{~d} \tau \int_{0}^{\tau}(\tilde{G}(\tau-s) w \Gamma(f, f)(s))\left(\tau, x^{\prime}, u\right) \mathrm{d} s\right| \\
& \leq H_{13,1}+H_{13,2}+H_{13,3}
\end{aligned}
$$

where

$$
\begin{aligned}
H_{13,1}= & \int_{\max \left\{t_{1}, 0\right\}}^{t} \int_{0}^{\tau} \int_{\mathbb{R}^{3}}\left|k_{w}^{c}(v, u)\right| \mathbf{1}_{\left\{t_{1}^{\prime} \leq s\right\}} I(t, s)\left|w \Gamma(f, f)\left(s, x^{\prime}-(\tau-s) u, u\right)\right| \mathrm{d} u \mathrm{~d} s \mathrm{~d} \tau, \\
H_{13,2}= & \int_{\max \left\{t_{1}, 0\right\}}^{t} \int_{0}^{\tau} \int_{\mathbb{R}^{3}} \int_{\max \left\{t_{1}^{\prime}, s\right\}}^{\tau} I\left(t, \tau^{\prime}\right)\left|k_{w}^{c}(v, u)\right| \mathrm{d} \tau^{\prime} \mathrm{d} u \mathrm{~d} s \mathrm{~d} \tau \\
& \quad \times \int_{\mathbb{R}^{3}}\left|k_{w}^{c}\left(u, u^{\prime}\right)\left(\tilde{G}\left(\tau^{\prime}-s\right) w \Gamma(f, f)(s)\right)\left(\tau^{\prime}, x^{\prime}-\left(\tau-\tau^{\prime}\right) u, u^{\prime}\right)\right| \mathrm{d} u^{\prime}, \\
H_{13,3}= & \left|H_{14}\right| \cdot \int_{\max \left\{t_{1}, 0\right\}}^{t} \int_{\mathbb{R}^{3}} I(t, \tau)\left|k_{w}^{c}(v, u)\right| \mathrm{d} u \mathrm{~d} \tau .
\end{aligned}
$$


Similar as before, we have

$$
\begin{aligned}
\left|H_{13,3}\right| & \leq\left|H_{14}\right| \cdot \int_{\max \left\{t_{1}, 0\right\}}^{t} I(t, \tau)\left\{\mathbf{1}_{\left\{|v| \leq d_{\Omega}\right\}}+\mathbf{1}_{\left\{|v|>d_{\Omega}\right\}}\right\} \mathrm{d} \tau \int_{\mathbb{R}^{3}}\left|k_{w}^{c}(v, u)\right| \mathrm{d} u \\
& \leq C\left|H_{14}\right| \cdot \int_{\max \left\{t_{1}, 0\right\}}^{t} e^{-\bar{\nu}_{0}(t-\tau)} \mathrm{d} \tau \leq C H_{14} .
\end{aligned}
$$

For $H_{13,1}$, we have from (2.20) and (2.21) that

$$
\begin{aligned}
\left|H_{13,1}\right| \leq & C \int_{\max \left\{t_{1}, 0\right\}}^{t} \mathrm{~d} \tau \int_{\mathbb{R}^{3}}\left|k_{w}^{c}(v, u)\right| \mathrm{d} u \int_{0}^{\tau} \mathbf{1}_{\left\{t_{1}^{\prime} \leq s\right\}} e^{-\bar{\nu}_{0}(t-s)}\|h(s)\|_{L^{\infty}} \mathrm{d} s \\
& \times\left(\int_{\mathbb{R}^{3}}\left|h\left(s, x^{\prime}-(\tau-s) u, u^{\prime}\right)\right|^{p}\left\langle u^{\prime}\right\rangle^{-4-p(\beta-4)} \mathrm{d} u^{\prime}\right)^{1 / p} .
\end{aligned}
$$

Now we divide the estimates by the following cases.

Case 1. $|v| \geq N$. We have from (2.14) that

$$
\left|H_{13,1}\right| \leq \frac{C}{N} \sup _{0 \leq s \leq t}\|h(s)\|_{L^{\infty}}^{2} .
$$

Case 2. $|v| \leq N,|u| \geq 2 N$. In this case, we have $|v-u| \geq N$, so that by (2.14),

$$
\int_{\{|u| \leq 2 N\}}\left|k_{w}^{c}(v, u)\right| \mathrm{d} u \leq e^{-\frac{N^{2}}{32}} \int_{\{|u| \leq 2 N\}}\left|k_{w}^{c}(v, u) e^{\frac{|v-u|^{2}}{32}}\right| \mathrm{d} u \leq C e^{-\frac{N^{2}}{32}} .
$$

It then follows that

$$
\left|H_{13,1}\right| \leq C e^{-\frac{N^{2}}{32}} \sup _{0 \leq s \leq t}\|h(s)\|_{L^{\infty}}^{2} .
$$

Case 3. $|v| \leq N,|u| \leq 2 N,\left|u^{\prime}\right|>N$. By $\beta>4$, it holds that

$$
\left|H_{13,1}\right| \leq \frac{C}{N^{\beta-4}} \sup _{0 \leq s \leq t}\|h(s)\|_{L^{\infty}}^{2} .
$$

Case 4. $|v| \leq N,|u| \leq 2 N,\left|u^{\prime}\right| \leq N, \tau-1 / N<s \leq \tau$. It is straightforward to see that

$$
\left|H_{13,1}\right| \leq \frac{C}{N} \sup _{0 \leq s \leq t}\|h(s)\|_{L^{\infty}}^{2} .
$$

Case 5. $|v| \leq N,|u| \leq 2 N, \mid u^{\prime} \leq N, 0 \leq s \leq \tau-1 / N$. By Hölder's inequality, we have

$$
\begin{aligned}
\left|H_{13,1}\right| \leq C_{N} & \sup _{0 \leq s \leq t}\|h(s)\|_{L^{\infty}} \cdot \int_{\max \left\{t_{1}, 0\right\}}^{t} \int_{0}^{\tau-\frac{1}{N}} e^{-\bar{\nu}_{0}(t-s)} \mathrm{d} s \mathrm{~d} \tau\left(\int_{\{|u| \leq 2 N\}}\left|k_{w}^{c}(v, u)\right|^{p^{\prime}} \mathrm{d} u\right)^{1 / p^{\prime}} \\
& \cdot\left(\int_{\{|u| \leq 2 N\}} \int_{\left\{\left|u^{\prime}\right| \leq N\right\}} \mathbf{1}_{\left\{t_{1}^{\prime} \leq s\right\}}\left|f\left(s, x^{\prime}-(\tau-s) u, u^{\prime}\right)\right|^{p} \mathrm{~d} u \mathrm{~d} u^{\prime}\right)^{1 / p} \\
\leq & C_{N, m} \sup _{0 \leq s \leq t}\|h(s)\|_{L^{\infty}} \cdot \sup _{0 \leq s \leq t}\|f(s)\|_{L^{p}} \leq \frac{C}{N} \sup _{0 \leq s \leq t}\|h(s)\|_{L^{\infty}}^{2}+C_{N, m} \sup _{0 \leq s \leq t}\|f(s)\|_{L^{p}}^{2} .
\end{aligned}
$$

Collecting the estimates for these cases, we have

$$
\left|H_{13,1}\right| \leq C\left(\frac{1}{N}+\frac{1}{N^{\beta-4}}\right) \sup _{0 \leq s \leq t}\|h(s)\|_{L^{\infty}}^{2}+C_{N, m} \sup _{0 \leq s \leq t}\|f(s)\|_{L^{p}}^{2} .
$$


Similarly, for $H_{13,2}$, we have

$$
\begin{aligned}
\left|H_{13,2}\right| \leq & \int_{\max \left\{t_{1}, 0\right\}}^{t} \int_{0}^{\tau} \mathrm{d} s \mathrm{~d} \tau \int_{s}^{\tau} e^{-\bar{\nu}_{0}\left(t-\tau^{\prime}\right)} \mathrm{d} \tau^{\prime} \\
& \times \int_{\mathbb{R}^{3}} \int_{\mathbb{R}^{3}}\left|k_{w}^{c}(v, u) k_{w}^{c}\left(u, u^{\prime}\right) \mathbf{1}_{\left\{\max \left\{t_{1}^{\prime}, s\right\} \leq \tau^{\prime} \leq \tau\right\}} Z\left(\tau^{\prime}, x^{\prime}-\left(\tau-\tau^{\prime}\right) u, u^{\prime}\right)\right| \mathrm{d} u \mathrm{~d} u^{\prime} \\
\leq & \frac{C}{N} \sup _{0 \leq s \leq t}\|h(s)\|_{L^{\infty}}^{2}+C_{N} \int_{\max \left\{t_{1}, 0\right\}}^{t} \int_{0}^{\tau} \mathrm{d} s \mathrm{~d} \tau \int_{s}^{\tau-\frac{1}{N}} e^{-\bar{\nu}_{0}\left(t-\tau^{\prime}\right)} \mathrm{d} \tau^{\prime} \\
& \times\left(\int_{\{|u| \leq 2 N\}} \int_{\left\{\left|u^{\prime}\right| \leq 3 N\right\}}\left|k_{w}^{c}(v, u) k_{w}^{c}\left(u, u^{\prime}\right)\right|^{p^{\prime}} \mathrm{d} u \mathrm{~d} u^{\prime}\right)^{1 / p^{\prime}} \\
& \times\left(\int_{\{|u| \leq 2 N\}} \int_{\left\{\left|u^{\prime}\right| \leq 3 N\right\}} \mathbf{1}_{\left\{\max \left\{t_{1}^{\prime}, s\right\} \leq \tau^{\prime} \leq \tau\right\}}\left|\frac{Z}{w}\left(\tau^{\prime}, x^{\prime}-\left(\tau-\tau^{\prime}\right) u, u^{\prime}\right)\right|^{p} \mathrm{~d} u \mathrm{~d} u^{\prime}\right)^{1 / p} \\
\leq & \frac{C}{N} \sup _{0 \leq s \leq t}\|h(s)\|_{L^{\infty}}^{2}+C_{N, m} \int_{\max \left\{t_{1}, 0\right\}}^{t} \int_{0}^{\tau} \mathrm{d} s \mathrm{~d} \tau \int_{s}^{\tau-\frac{1}{N}} e^{-\bar{\nu}_{0}\left(t-\tau^{\prime}\right)}\left\|\frac{Z\left(\tau^{\prime}\right)}{w}\right\|_{L^{p}} \mathrm{~d} \tau^{\prime} \\
\leq & \frac{C}{N} \sup _{0 \leq s \leq t}\|h(s)\|_{L^{\infty}}^{2}+C_{N, T_{0}, m} \sup _{0 \leq s \leq t}\|f(s)\|_{L^{p}}^{2} .
\end{aligned}
$$

Here we have used (5.23) in the last inequality. Substituting (5.30), (5.31) and (5.32) into (5.29), we have

$$
\begin{aligned}
& \int_{0}^{t}|(\tilde{G}(t-s) w \Gamma(f, f)(s))(t, x, v)| \mathrm{d} s \\
& \quad \leq \int_{0}^{t} \mathbf{1}_{\left\{t_{1} \leq s\right\}} I(t, s) w \Gamma(f, f)(s, x-(t-s) v, v) \mathrm{d} s \\
& \quad+C T_{0}^{5 / 2}\left\{m^{3+\kappa}+\delta+2^{-\hat{C}_{4} T_{0}^{5 / 4}}+\frac{1}{N}+\frac{1}{N^{\beta-4}}\right\} \bar{M}^{2}+C_{N, T_{0}, m} \sup _{0 \leq s \leq t}\|f(s)\|_{L^{p}}^{2} .
\end{aligned}
$$

Then (5.10) naturally follows from (5.12), (5.13) and (5.33). Therefore, the proof of Lemma 5.3) is complete.

Lemma 5.4. Under the assumption (5.1), there exists a constant $C>0$ independent of $t$, such that, for any $0 \leq t \leq \min \left\{T, T_{0}\right\}$, it holds that:

$$
\begin{aligned}
\|h(t)\|_{L^{\infty}}+|h(t)|_{L^{\infty}(\gamma)} \leq & C M_{0} e^{-\lambda_{0} t^{\alpha}}\left(1+\int_{0}^{t}\|h(\tau)\|_{L^{\infty}} \mathrm{d} \tau\right) \\
& +C T_{0}^{5 / 2}\left\{m^{3+\kappa}+\delta+2^{-\hat{C}_{4} T_{0}^{5 / 4}}+\frac{1}{N}+\frac{1}{N^{\beta-4}}\right\} \cdot\left\{\bar{M}+\bar{M}^{3}\right\} \\
& +C_{N, T_{0}, m}\left\{\sup _{0 \leq s \leq t}\|f(s)\|_{L^{p}}+\sup _{0 \leq s \leq t}\|f(s)\|_{L^{p}}^{3}\right\} .
\end{aligned}
$$

Here the positive constants $T_{0}$ and $N$ can be chosen arbitrarily large and $m$ can be chosen arbitrarily small. 
Proof. We denote $x^{\prime}:=x-(t-s) v$ and $t_{1}^{\prime}:=t_{1}\left(s, x^{\prime}, u\right)$. It suffices to consider the last term on the right-hand side of (5.10). By (2.20) and (2.21), it holds that

$$
\begin{aligned}
& \int_{\max \left\{t_{1}, 0\right\}}^{t} I(t, s)|w \Gamma(f, f)(s, x-(t-s) v, u)| \mathrm{d} u \mathrm{~d} s \\
& \leq \int_{\max \left\{t_{1}, 0\right\}}^{t} e^{-\bar{\nu}_{0}(t-s)}\|w f(s)\|_{L^{\infty}} \mathrm{d} s\left(\int_{\mathbb{R}^{3}}|h(s, x-(t-s) v, u)|^{p}\langle u\rangle^{-4-p(\beta-4)}\right)^{1 / p} \\
& \leq \frac{C}{N^{\beta-4}} \sup _{0 \leq s \leq t}\|h(s)\|_{L^{\infty}}^{2} \\
& \quad+C \int_{\max \left\{t_{1}, 0\right\}}^{t} e^{-\bar{\nu}_{0}(t-s)}\|w f(s)\|_{L^{\infty}} \mathrm{d} s \times\left(\int_{\{|u| \leq N\}}\left|h\left(s, x^{\prime}, u\right)\right|^{p}\langle u\rangle^{-4-p(\beta-4)}\right)^{1 / p} .
\end{aligned}
$$

Notice that $x^{\prime}:=x-(t-s) v \in \Omega$. Then applying (5.10) to $h\left(s, x^{\prime}, u\right)$, we have

$$
\int_{\max \left\{t_{1}, 0\right\}}^{t} I(t, s)|w \Gamma(f, f)(s, x-(t-s) v, u)| \mathrm{d} u \mathrm{~d} \tau \leq \frac{C}{N^{\beta-4}} \sup _{0 \leq s \leq t}\|h(s)\|_{L^{\infty}}^{2}+R_{1}+R_{2},
$$

where we have defined

$$
R_{1}=C \int_{\max \left\{t_{1}, 0\right\}}^{t} e^{-\bar{\nu}_{0}(t-s)}\|w f(s)\|_{L^{\infty} S(s) \mathrm{d} s}
$$

and

$$
\begin{aligned}
R_{2}=C & \int_{\max \left\{t_{1}, 0\right\}}^{t} e^{-\bar{\nu}_{0}(t-s)}\|w f(s)\|_{L^{\infty}} \mathrm{d} s \times\left(\int_{\{|u| \leq N\}}\langle u\rangle^{-4-p(\beta-4)} \mathrm{d} u\right. \\
& \left.\times\left\{\int_{\max \left\{t_{1}^{\prime}, 0\right\}}^{s} e^{-\bar{\nu}_{0}\left(s-s^{\prime}\right)}\left|w \Gamma(f, f)\left(s^{\prime}, x^{\prime}-\left(s-s^{\prime}\right) u, u\right)\right| \mathrm{d} \tau^{\prime}\right\}^{p}\right)^{1 / p} .
\end{aligned}
$$

A direct computation shows that

$$
\begin{aligned}
\left|R_{1}\right| \leq & C \int_{\max \left\{t_{1}, 0\right\}}^{t} e^{-\bar{\nu}_{0}(t-s)}\|w f(s)\|_{L^{\infty}} e^{-\lambda_{0} s^{\alpha}}\left\|h_{0}\right\|_{L^{\infty}} \mathrm{d} s \\
& +C T_{0}^{5 / 2} \sup _{0 \leq s \leq t}\|h(s)\|_{L^{\infty}} \times\left\{m^{3+\kappa}+\delta+2^{-T_{0}}+\frac{1}{N}+\frac{1}{N^{\beta-4}}\right\} \cdot\left\{\bar{M}+\bar{M}^{2}\right\} \\
& +C_{N, T_{0}, m} \sup _{0 \leq s \leq t}\|h(s)\|_{L^{\infty}} \cdot\left\{\sup _{0 \leq s \leq t}\|f(s)\|_{L^{p}}+\sup _{0 \leq s \leq t}\|f(s)\|_{L^{p}}^{2}\right\} \\
\leq & C M_{0} e^{-\lambda_{0} t^{\alpha}} \int_{0}^{t}\|h(s)\|_{L^{\infty}} \mathrm{d} s+C T_{0}^{5 / 2}\left\{m^{3+\kappa}+\delta+2^{-T_{0}}+\frac{1}{N}+\frac{1}{N^{\beta-4}}\right\} \cdot\left\{\bar{M}+\bar{M}^{3}\right\} \\
& +C_{N, T_{0}, m}\left\{\sup _{0 \leq s \leq t}\|f(s)\|_{L^{p}}+\sup _{0 \leq s \leq t}\|f(s)\|_{L^{p}}^{3}\right\} .
\end{aligned}
$$


For $R_{2}$, using (2.20) and (2.21) again, we have

$$
\begin{aligned}
\left|R_{2}\right| \leq & C \sup _{0 \leq s \leq t}\|h(s)\|_{L^{\infty}}^{2} \cdot \int_{\max \left\{t_{1}, 0\right\}}^{t} e^{-\bar{\nu}_{0}(t-s)} \mathrm{d} s \\
& \times\left(\int_{|u| \leq N} \int_{\max \left\{t_{1}^{\prime}, 0\right\}}^{\tau} \int_{\mathbb{R}^{3}} e^{-\bar{\nu}_{0}\left(s-s^{\prime}\right)}\left|h\left(s^{\prime}, x^{\prime}-\left(s-s^{\prime}\right) u, u^{\prime}\right)\right|^{p}\left[\langle u\rangle\left\langle u^{\prime}\right\rangle\right]^{-4-p(\beta-4)}\right)^{1 / p} \\
\leq & C\left(\frac{1}{N}+\frac{1}{N^{\beta-4}}\right) \sup _{0 \leq s \leq t}\|h(s)\|_{L^{\infty}}^{3}+C_{N} \sup _{0 \leq s \leq t}\|h(s)\|_{L^{\infty}}^{2} \cdot \int_{\max \left\{t_{1}, 0\right\}}^{t} e^{-\bar{\nu}_{0}(t-s)} \mathrm{d} s \\
& \times\left(\int_{0}^{s-\frac{1}{N}} e^{-\bar{\nu}_{0}\left(s-s^{\prime}\right)} \mathrm{d} s^{\prime} \int_{|u| \leq N} \int_{\left|u^{\prime}\right| \leq N} \mathbf{1}_{\max \left\{t_{1}^{\prime}, 0\right\} \leq s^{\prime} \leq s}\left|f\left(s^{\prime}, x^{\prime}-\left(s-s^{\prime}\right) u, u^{\prime}\right)\right|^{p} \mathrm{~d} u \mathrm{~d} u^{\prime}\right)^{1 / p} \\
\leq & C\left(\frac{1}{N}+\frac{1}{N^{\beta-4}}\right) \sup _{0 \leq s \leq t}\|h(s)\|_{L^{\infty}}^{3}+C_{N} \sup _{0 \leq s \leq t}\|f(s)\|_{L^{p}}^{3} .
\end{aligned}
$$

Here we have used the change of variable $u \rightarrow y^{\prime}:=x^{\prime}-\left(s-s^{\prime}\right) u$. Then from combining (5.10), (5.35), (5.36) and (5.37), (5.34) follows. Therefore, the proof of Lemma 5.4 is complete.

\subsection{Proof of Theorem 1.3, Let}

$$
\mathcal{E}(t):=1+\int_{0}^{t}\left(\|h(s)\|_{L^{\infty}}+|h(s)|_{L^{\infty}(\gamma)}\right) \mathrm{d} s .
$$

Then it holds from (5.34) that

$$
\|h(t)\|_{L^{\infty}}+|h(t)|_{L^{\infty}(\gamma)}=\mathcal{E}^{\prime}(t) \leq C M_{0} e^{-\lambda_{0} t^{\alpha}} \mathcal{E}(t)+\mathcal{D},
$$

where

$$
\begin{aligned}
\mathcal{D}:=C T_{0}^{5 / 2}\left\{m^{3+\kappa}+\delta+\frac{1}{N}+\frac{1}{N^{\beta-4}}\right\} \bar{M}^{3}+ & 2^{-T_{0}} \bar{M}^{3} \\
& +C_{N, T_{0}, m}\left\{e^{C_{3} \bar{M} T_{0}}\left\|f_{0}\right\|_{L^{p}}+\left(e^{C_{3} \bar{M} T_{0}}\left\|f_{0}\right\|_{L^{p}}\right)^{3}\right\} .
\end{aligned}
$$

From (5.38), we have

$$
\mathcal{E}(t) \leq \mathcal{E}(0) e^{C M_{0} \int_{0}^{t} e^{-\lambda_{0} s^{\alpha}} \mathrm{d} s}+\mathcal{D} \cdot \int_{0}^{t} e^{C M_{0} \int_{s}^{t} e^{-\lambda_{0} \tau^{\alpha}} \mathrm{d} \tau} \mathrm{d} s \leq(1+\mathcal{D} t) e^{C M_{0}} .
$$

Substituting (5.39) into (5.38), we have

$$
\|h(t)\|_{L^{\infty}}+|h(t)|_{L^{\infty}(\gamma)} \leq C M_{0} e^{C M_{0}}(1+\mathcal{D} t) e^{-\lambda_{0} t^{\alpha}}+\mathcal{D} \leq e^{C M_{0}}(1+\mathcal{D}) e^{-\frac{\lambda_{0} t^{\alpha}}{2}}+\mathcal{D} .
$$

Take $\bar{M}=2 e^{C M_{0}}, \tilde{\varepsilon}=\min \left\{\varepsilon_{0},\left(2 C_{0}\right)^{-1}\right\}$ where $C_{0}$ and $\varepsilon_{0}$ are the same as ones in Theorem 1.2 , and

$$
T_{0}:=\max \left\{3\left(\log _{2} \bar{M}+1\right)+\left|\log _{2} \tilde{\varepsilon}\right|,\left(\frac{2(\log 4 \bar{M}+|\log \tilde{\varepsilon}|)}{\lambda_{0}}\right)^{1 / \alpha}\right\},
$$

such that $2^{-T_{0}} \bar{M}^{3} \leq \frac{\tilde{\varepsilon}}{8}$ and $\bar{M} e^{-\frac{\lambda_{0} T_{0}^{\alpha}}{2}} \leq \tilde{\frac{\varepsilon}{4}}$. Then it holds that

$$
C T_{0}^{5 / 2} \delta \bar{M}^{3} \leq C_{4}\left[|\log \tilde{\varepsilon}|^{\frac{5}{2 \alpha}}+1\right] e^{C_{4} M_{0}} \delta
$$

for some universal constant $C_{4}>1$. Let

$$
0<\delta \leq \delta_{0}<\left(\frac{\tilde{\varepsilon}}{16 C_{4}\left[|\log \tilde{\varepsilon}|^{\frac{5}{2 \alpha}}+1\right]}\right)^{2}
$$


and $0<M_{0} \leq \frac{|\log \delta|}{2 C_{4}}$. Then it is straightforward to see that $C T_{0}^{5 / 2} \delta \bar{M}^{3} \leq \frac{\tilde{\varepsilon}}{16}$. Now we take $0<m<1$ suitably small and $N$ suitably large, and finally take $\left\|f_{0}\right\|_{L^{p}} \leq \varepsilon_{1}$, with $\varepsilon_{1}>0$ sufficiently small, such that

$$
C T_{0}^{5 / 2}\left\{m^{3+\kappa}+\frac{1}{N}+\frac{1}{N^{\beta-4}}\right\} \bar{M}^{3}+C_{N, T_{0}, m}\left\{e^{C_{3} \bar{M} T_{0}}\left\|f_{0}\right\|_{L^{p}}+\left(e^{C_{3} \bar{M} T_{0}}\left\|f_{0}\right\|_{L^{p}}\right)^{3}\right\} \leq \frac{\tilde{\varepsilon}}{16} .
$$

Therefore, we have $\mathcal{D} \leq \frac{\tilde{\varepsilon}}{4}$. From (5.40), it holds, for any $t \in\left[0, T_{0}\right]$, that

$$
\|h(t)\|_{L^{\infty}}+|h(t)|_{L^{\infty}(\gamma)} \leq e^{C M_{0}}(1+\mathcal{D})+\mathcal{D} \leq\left(1+\frac{\tilde{\varepsilon}}{4}\right) e^{C M_{0}}+\frac{\tilde{\varepsilon}}{4} \leq \frac{3 \bar{M}}{4} .
$$

Notice that at $t=T_{0}$, we have from (5.40) that

$$
\left\|h\left(T_{0}\right)\right\|_{L^{\infty}} \leq e^{C M_{0}}(1+\mathcal{D}) e^{-\frac{\lambda_{0} T_{0}^{\alpha}}{2}}+\mathcal{D} \leq \frac{\tilde{\varepsilon}}{2} .
$$

Then from (1.17), we have, for $t>T_{0}$,

$$
\|h(t)\|_{L^{\infty}}+|h(t)|_{L^{\infty}(\gamma)} \leq C_{0}\left\|h\left(T_{0}\right)\right\|_{L^{\infty}} \leq C_{0} \tilde{\varepsilon} \leq \frac{3 \bar{M}}{4} .
$$

A combination of (5.41) and (5.42) justifies that the a priori assumption (5.1) can be closed by our choice. Notice that the local existence has been established in Proposition 6.2. Then the global existence of the solution follows from a standard continuity argument. For large time behavior, it holds, for $t \in\left[0, T_{0}\right]$, that

$$
\|h(t)\|_{L^{\infty}}+|h(t)|_{L^{\infty}(\gamma)} \leq \bar{M} \leq e^{2 C M_{0}} e^{\lambda_{0} T_{0}^{\alpha}} e^{-\lambda_{0} t^{\alpha}} \leq C_{5} e^{C_{5} M_{0}} e^{-\lambda_{0} t^{\alpha}},
$$

for some constant $C_{5}>1$. For $t>T_{0}$, it holds from (1.17) that

$$
\|h(t)\|_{L^{\infty}}+|h(t)|_{L^{\infty}(\gamma)} \leq C_{0} e^{-\lambda_{0}\left(t-T_{0}\right)^{\alpha}}\left\|h\left(T_{0}\right)\right\|_{L^{\infty}} \leq C_{0} C_{5} e^{C_{5} M_{0}} e^{-\lambda_{0} t^{\alpha}} .
$$

By taking $C_{2}=C_{0} C_{5}$, (1.23) follows from (5.43) and (5.44). Therefore, the proof of Theorem 1.3 is complete.

\section{Appendix}

\subsection{An iteration lemma.}

Lemma 6.1. Consider a sequence $\left\{a_{i}\right\}_{i=0}^{\infty}$ with each $a_{i} \geq 0$. For any fixed $k \in \mathbb{N}_{+}$, we denote

$$
A_{i}^{k}=\max \left\{a_{i}, a_{i+1}, \cdots, a_{i+k}\right\} .
$$

(1) Assume $D \geq 0$. If $a_{i+1+k} \leq \frac{1}{8} A_{i}^{k}+D$ for $i=0,1, \cdots$, then it holds that

$$
A_{i}^{k} \leq\left(\frac{1}{8}\right)^{\left[\frac{i}{k+1}\right]} \cdot \max \left\{A_{0}^{k}, A_{1}^{k}, \cdots, A_{k}^{k}\right\}+\frac{8+k}{7} D, \text { for } i \geq k+1 .
$$

(2) Let $0 \leq \eta<1$ with $\eta^{k+1} \geq \frac{1}{4}$. If $a_{i+1+k} \leq \frac{1}{8} A_{i}^{k}+C_{k} \cdot \eta^{i+k+1}$ for $i=0,1, \cdots$, then it holds that

$$
A_{i}^{k} \leq\left(\frac{1}{8}\right)^{\left[\frac{i}{k+1}\right]} \cdot \max \left\{A_{0}^{k}, A_{1}^{k}, \cdots, A_{k}^{k}\right\}+2 C_{k} \frac{8+k}{7} \eta^{i+k}, \quad \text { for } \quad i \geq k+1 .
$$

Proof. We first show (6.1). By iteration in $i \geq 0$, we obtain that

$$
\begin{aligned}
a_{i+1+2 k} & \leq \frac{1}{8} A_{i+k}^{k}+D=\frac{1}{8} \max \left\{a_{i+2 k}, a_{i+2 k-1}, \cdots, a_{i+k}\right\}+D \\
& \leq \frac{1}{8} \max \left\{a_{i+2 k}, A_{i+k-1}^{k}\right\}+D \leq \frac{1}{8} \max \left\{\frac{1}{8} A_{i+k-1}^{k}+D, A_{i+k-1}^{k}\right\}+D \\
& \leq \frac{1}{8} A_{i+k-1}^{k}+\left(1+\frac{1}{8}\right) D \cdots \leq \frac{1}{8} A_{i}^{k}+\left(1+\frac{k}{8}\right) D .
\end{aligned}
$$


Similarly, for all $j=0,1, \cdots, k-1$, we also have

$$
a_{i+1+j+k} \leq \frac{1}{8} A_{i}^{k}+\frac{8+k}{8} D .
$$

Therefore, for $1 \leq \frac{i}{k+1} \in \mathbb{N}_{+}$, it follows from (6.3) and (6.4) that

$$
\begin{aligned}
A_{i+1+k}^{k} & =\max \left\{a_{i+1+2 k}, a_{i+2 k}, \cdots, a_{i+1+k}\right\} \\
& \leq \frac{1}{8} A_{i}^{k}+\frac{8+k}{8} D \leq\left(\frac{1}{8}\right)^{2} A_{i-2(k+1)}^{k}+\frac{1}{8} \frac{8+k}{8} D+\frac{8+k}{8} D \\
& =\left(\frac{1}{8}\right)^{2} A_{i-2(k+1)}^{k}+\frac{8+k}{8}\left(1+\frac{1}{8}\right) D=\cdots \\
& \leq\left(\frac{1}{8}\right)^{\frac{i}{k+1}} A_{0}^{k}+\frac{8+k}{8}\left(1+\frac{1}{8}+\left(\frac{1}{8}\right)^{2}+\cdots\right) D \\
& \leq\left(\frac{1}{8}\right)^{\frac{i}{k+1}} A_{0}^{k}+\frac{8+k}{7} D .
\end{aligned}
$$

If $\frac{i}{k+1} \notin \mathbb{N}_{+}$and $i=(k+1)\left[\frac{i}{k+1}\right]+j$ for some $1 \leq j \leq k$, then by similar arguments we have

$$
A_{i+1+k}^{k} \leq\left(\frac{1}{8}\right)^{\left[\frac{i}{k+1}\right]+1} A_{j}^{k}+\frac{8+k}{7} D
$$

Hence, from (6.5) and (6.6), we complete the proof of (6.1).

It remains to show (6.2). Noting $\eta<1$ and by similar arguments as in (6.3) and (6.4), we can get

$$
a_{i+j+k+1} \leq \frac{1}{8} A_{i}^{k}+C_{k}\left(1+\frac{k}{8}\right) \eta^{i+k+1}, \text { for } 0 \leq j \leq k .
$$

Hence, for $1 \leq \frac{i}{k+1} \in \mathbb{N}_{+}$, noting $\frac{1}{8} \cdot \eta^{-k-1} \leq \frac{1}{2}$ and using (6.7), then we have

$$
\begin{aligned}
& A_{i+k+1}^{k}=\max \left\{a_{i+2 k+1}, \cdots, a_{i+k+1}\right\} \leq \frac{1}{8} A_{i}^{k}+C_{k}\left(1+\frac{k}{8}\right) \eta^{i+k+1} \\
& \leq \cdots \leq\left(\frac{1}{8}\right)^{\frac{i}{k+1}} A_{0}^{k}+C_{k}\left(1+\frac{k}{8}\right) \eta^{i+k+1} \cdot\left\{1+\frac{1}{8} \eta^{-k-1}+\left(\frac{1}{8} \eta^{-k-1}\right)^{2}+\cdots\right\} \\
& \leq\left(\frac{1}{8}\right)^{\frac{i}{k+1}} A_{0}^{k}+2 C_{k}\left(1+\frac{k}{8}\right) \eta^{i+k+1} .
\end{aligned}
$$

If $\frac{i}{k+1} \notin \mathbb{N}_{+}$and $i=(k+1)\left[\frac{i}{k+1}\right]+j$ for some $1 \leq j \leq k$, then by similar arguments as above, we have

$$
A_{i+k+1}^{k} \leq\left(\frac{1}{8}\right)^{\left[\frac{i}{k+1}\right]+1} A_{j}^{k}+2 C_{k}\left(1+\frac{k}{8}\right) \eta^{i+k+1} .
$$

Thus we prove (6.2) from (6.8) and (6.9). Therefore the proof of lemma 6.1] is complete.

\subsection{Local-in-time existence.}

Proposition 6.2. Let $w(v)$ be the weight function defined in (1.9). Assume

$$
\left|\theta-\theta_{0}\right|_{L^{\infty}(\partial \Omega)}=\delta \ll 1, \quad F_{0}(x, v)=F_{*}(x, v)+\mu^{\frac{1}{2}}(v) f_{0}(x, v) \geq 0
$$

and $\left\|w f_{0}\right\|_{L^{\infty}}:=M_{0}<\infty$. Then there exists a positive time

$$
\hat{t}:=\left[\hat{C}\left(1+M_{0}\right)\right]^{-1}
$$


such that the IBVP (1.1), (1.5) and (1.13) has a unique nonnegative solution

$$
F(t, x, v)=F_{*}(x, v)+\mu^{\frac{1}{2}}(v) f(t, x, v) \geq 0
$$

in $[0, \hat{t}]$ satisfying

$$
\sup _{0 \leq t \leq \hat{t}}\left\{\|w f(t)\|_{L^{\infty}}+|w f(t)|_{L^{\infty}(\gamma)}\right\} \leq 2 \tilde{C}\left(M_{0}+1\right) .
$$

Here $\tilde{C}>0$ and $\hat{C}>1$ are generic constants independent of $M_{0}$. Moreover, if the domain $\Omega$ is strictly convex, $\theta(x)$ is continuous over $\partial \Omega$, the initial data $F_{0}(x, v)$ is continuous except on $\gamma_{0}$ and satisfies (1.19) then the solution $F(t, x, v)$ is continuous in $[0, \hat{t}] \times\left\{\Omega \times \mathbb{R}^{3} \backslash \gamma_{0}\right\}$.

Proof. We consider the following iteration scheme:

$$
\left\{\begin{array}{l}
\partial_{t} F^{n+1}+v \cdot \nabla_{x} F^{n+1}+F^{n+1} \cdot R\left(F^{n}\right)=Q^{+}\left(F^{n}, F^{n}\right) \\
\left.F^{n+1}(t, x, v)\right|_{t=0}=F_{0}(x, v) \geq 0 \\
\left.F^{n+1}(t, x, v)\right|_{\gamma_{-}}=\mu_{\theta}(x, v) \int_{n(x) \cdot u>0} F^{n+1}(t, x, u)\{u \cdot n(x)\} \mathrm{d} u \\
F^{0}(t, x, v)=\mu(v)
\end{array}\right.
$$

where

$$
R\left(F^{n}\right)(t, x, v)=\int_{\mathbb{R}^{3} \times \mathbb{S}^{2}} B(v-u, \omega) F^{n}(t, x, u) \mathrm{d} u \mathrm{~d} \omega
$$

Let

$$
f^{n+1}(t, x, v)=\frac{F^{n+1}(t, x, v)-F_{*}(x, v)}{\mu^{\frac{1}{2}}(v)}, \quad h^{n+1}(t, x, v)=w f^{n+1}(t, x, v) .
$$

Then the equation of $h^{n+1}$ reads as

$$
\left\{\begin{array}{l}
\partial_{t} h^{n+1}+v \cdot \nabla_{x} h^{n+1}+h^{n+1} \cdot R\left(F^{n}\right)=w K_{*} f^{n}+w \Gamma^{+}\left(f^{n}, f^{n}\right), \\
\left.h^{n+1}(t, x, v)\right|_{t=0}=h_{0}(x, v), \\
\left.h^{n+1}\right|_{\gamma_{-}}=\frac{1}{\tilde{w}(v)} \int_{\{n(x) \cdot u>0\}} h^{n+1} \tilde{w}(u) \mathrm{d} \sigma(x)+w(v) \frac{\mu_{\theta}-\mu}{\sqrt{\mu}} \int_{n(x) \cdot u>0} h^{n+1} \tilde{w}(u) \mathrm{d} \sigma(x), \\
h^{0}(t, x, v)=-w f_{*}(x, v),
\end{array}\right.
$$

where we have denoted

$$
K_{*} f^{n}:=-\sqrt{\mu}^{-1}\left\{R\left(\sqrt{\mu} f^{n}\right) F_{*}+\Gamma^{+}\left(\sqrt{\mu} f^{n}, F_{*}\right)+\Gamma^{+}\left(F_{*}, \sqrt{\mu} f^{n}\right)\right\} .
$$

Now we shall use the induction on $n=0,1, \cdots$ to show that there exists a positive time $\hat{t}_{1}>0$, independent of $n$, such that (6.10) or equivalently (6.11) admits a unique mild solution on the time interval $\left[0, \hat{t}_{1}\right]$, and the following uniform bound and positivity hold true:

$$
\left\|h^{n}(t)\right\|_{L^{\infty}}+\left|h^{n}(t)\right|_{L^{\infty}(\gamma)} \leq 2 \tilde{C}\left[\left\|h_{0}\right\|_{L^{\infty}}+1\right],
$$

and

$$
F^{n}(t, x, v) \geq 0
$$

for $0 \leq t \leq \hat{t}=\left(\hat{C}\left\{1+\left\|h_{0}\right\|_{L^{\infty}}\right\}\right)^{-1}$ and suitably chosen constants $\tilde{C}>0$ and $\hat{C}$ independent of $t$. Thanks to the fact

$$
\left\|h^{0}(t)\right\|_{L^{\infty}}+\left|h^{0}(t)\right|_{L^{\infty}(\gamma)} \leq\left\|w f_{*}\right\|_{L^{\infty}}+\left|w f_{*}\right|_{L^{\infty}(\gamma)} \leq C \delta
$$


we see that (6.12) is obviously true for $n=0$. To proceed, we assume that (6.12) holds true up to $n \geq 0$. Since $F^{n} \geq 0$, it holds that $R\left(F^{n}\right) \geq 0$. Then by using a similar argument as in 19 , Lemma 3.4], one can construct the solution operator $G^{n}(t)$ to the following linear problem

$$
\left\{\begin{array}{l}
\partial_{t} h+v \cdot \nabla_{x} h+R\left(F^{n}\right) h=0, \quad t>0, \quad x \in \Omega, \quad v \in \mathbb{R}^{3}, \\
\left.h(t, x, v)\right|_{t=0}=h_{0}(x, v), \\
\left.h(t, x, v)\right|_{\gamma_{-}}=\frac{1}{\tilde{w}(v)} \int_{n(x) \cdot u>0} h(t, x, u) \tilde{w}(u) \mathrm{d} \sigma(x)+w(v) \frac{\mu_{\theta}-\mu}{\sqrt{\mu}} \int_{n(x) \cdot u>0} h(t, x, u) \tilde{w}(u) \mathrm{d} \sigma(x)
\end{array}\right.
$$

over $(0, \rho)$ for some universal constant $\rho>0$ independent of $n$, provided that $|\theta-1|_{L^{\infty}(\partial \Omega)}$ is sufficiently small. Moreover, $G^{n}(t)$ satisfies the following estimate

$$
\left\|G^{n}(t) h_{0}\right\|_{L^{\infty}}+\left|G^{n}(t) h_{0}\right|_{L^{\infty}(\gamma)} \leq C_{\rho}\left\|h_{0}\right\|_{L^{\infty}}
$$

Here the constant $C_{\rho}>0$ is independent of $n$. Then applying Duhamel's formula to (6.11), we have, for $0<t<\rho$, that

$$
h^{n+1}(t)=G^{n}(t, 0) h_{0}+\int_{0}^{t} G^{n}(t, s)\left[w K_{*} f^{n}(s)+w \Gamma^{+}\left(f^{n}, f^{n}\right)(s)\right] \mathrm{d} s .
$$

Taking $L^{\infty}$-norm on the both sides of (6.15) and using (2.16) and (6.14), we have

$$
\begin{aligned}
& \left\|h^{n+1}(t)\right\|_{L^{\infty}}+\left|h^{n+1}(t)\right|_{L^{\infty}(\gamma)} \\
& \leq C_{\rho}\left\|h_{0}\right\|_{L^{\infty}}+C_{\rho} \int_{0}^{t}\left\|w K_{*} f^{n}(s)\right\|_{L^{\infty}}+\left\|w \Gamma^{+}\left(f^{n}, f^{n}\right)(s)\right\|_{L^{\infty}} \mathrm{d} s \\
& \leq C\left\|h_{0}\right\|_{L^{\infty}}+C \int_{0}^{t}\left\|h^{n}(s)\right\|_{L^{\infty}}+\left\|h^{n}(s)\right\|_{L^{\infty}}^{2} \mathrm{~d} s \\
& \leq C_{3}\left\|h_{0}\right\|_{L^{\infty}}+C_{3} t \cdot\left\{\sup _{0 \leq s \leq t}\left\|h^{n}(s)\right\|_{L^{\infty}}+\sup _{0 \leq s \leq t}\left\|h^{n}(s)\right\|_{L^{\infty}}^{2}\right\},
\end{aligned}
$$

for some constants $C_{3}>1$. Now we take $\tilde{C}=C_{3}$ and $\hat{C}=8 C_{3}^{2}$. Then by the induction hypothesis (6.12), for any $0<t<\hat{t}$, it follows from (6.16) that

$$
\begin{aligned}
\left\|h^{n+1}(t)\right\|_{L^{\infty}}+\left|h^{n+1}(t)\right|_{L^{\infty}(\gamma)} & \leq C_{3}\left\{\left\|h_{0}\right\|_{L^{\infty}}+1\right\} \cdot\left\{1+2 C_{3} t\left[1+2 C_{3}\right] \cdot\left[1+\left\|h_{0}\right\|_{L^{\infty}}\right]\right\} \\
& \leq 2 C_{3}\left\{\left\|h_{0}\right\|_{L^{\infty}}+1\right\} .
\end{aligned}
$$

This then proves (6.12) for $n+1$. Next we show the non-negativity (6.13) for $n+1$. We denote that

$$
I^{n}(t, s):=\exp \left\{-\int_{s}^{t}\left[R\left(F^{n}\right)\right]\left(\tau, X_{c l}(\tau), V_{c l}(\tau)\right) \mathrm{d} \tau\right\}
$$

and

$$
\mathrm{d} \Sigma_{l}^{n}(\tau):=\left\{\prod_{j=l+1}^{k-1} \mathrm{~d} \sigma_{j}\right\} \cdot I^{n}\left(t_{l}, \tau\right)\left[v_{l} \cdot n\left(v_{l}\right)\right] \mathrm{d} v_{l} \cdot \prod_{j=1}^{l-1}\left\{I^{n}\left(t_{j}, t_{j+1}\right) \mu_{\theta}\left(x_{j+1}, v_{j}\right)\left[v_{j} \cdot n\left(x_{j}\right)\right] \mathrm{d} v_{j}\right\}
$$


Then we have the following mild formulation for $F^{n+1}$ :

$$
\begin{aligned}
F^{n+1}(t, x, v)= & \mathbf{1}_{\left\{t_{1} \leq 0\right\}}\left\{I^{n}(t, 0) F_{0}(x-v t, v)+\int_{0}^{t} I^{n}(t, s) Q^{+}\left(F^{n}, F^{n}\right)(s, x-v(t-s), v) \mathrm{d} s\right\} \\
& +\mathbf{1}_{\left\{t_{1}>0\right\}} \mu_{\theta}\left(x_{1}, v\right) I^{n}\left(t, t_{1}\right)\left\{\int_{\Pi_{j=1}^{k-1} \mathcal{V}_{j}} \sum_{l=1}^{k-1} \mathbf{1}_{\left\{t_{l+1} \leq 0<t_{l}\right\}} F_{0}(x-v t, v) \mathrm{d} \Sigma_{l}^{n}(0)\right. \\
& +\int_{\Pi_{j=1}^{k-1} \mathcal{V}_{j}} \sum_{l=1}^{k-1} \int_{0}^{t_{l}} \mathbf{1}_{\left\{t_{l+1} \leq 0<t_{l}\right\}} Q^{+}\left(F^{n}, F^{n}\right)\left(\tau, X_{c l}(\tau), V_{c l}(\tau)\right) \mathrm{d} \Sigma_{l}^{m}(\tau) \mathrm{d} \tau \\
& \left.+\int_{\Pi_{j=1}^{k-1} \mathcal{V}_{j}} \sum_{l=1}^{k-1} \int_{t_{l+1}}^{t_{l}} \mathbf{1}_{\left\{t_{l+1} \leq 0<t_{l}\right\}} Q^{+}\left(F^{n}, F^{n}\right)\left(\tau, X_{c l}(\tau), V_{c l}(\tau)\right) \mathrm{d} \Sigma_{l}^{m}(\tau) \mathrm{d} \tau\right\} \\
& +\mathbf{1}_{\left\{t_{1}>0\right\}} \mu_{\theta}\left(x_{1}, v\right) I^{n}\left(t, t_{1}\right) \int_{\Pi_{j=1}^{k-1} \mathcal{V}_{j}} \mathbf{1}_{\left\{t_{k}>0\right\}} F^{n+1}\left(t_{k}, x_{k}, v_{k-1}\right) \mathrm{d} \Sigma_{k-1}^{n}\left(t_{k}\right), \quad(6 .
\end{aligned}
$$

for $t>0, x \in \bar{\Omega} \times \mathbb{R}^{3} \backslash \gamma_{0} \cup \gamma_{-}$and integer $k \geq 1$. From (6.12), it holds that

$$
\left|F^{n+1}(t, x, v)\right|=\left|F_{*}(x, v)+\mu^{\frac{1}{2}}(v) \frac{h^{n+1}(t, x, v)}{w(v)}\right| \leq C\left(1+\left\|h_{0}\right\|_{L^{\infty}}\right) \mu^{\frac{1}{2}}(v),
$$

for some constant $C>0$. Furthermore, a direct computation shows that

$$
0<\mu_{\theta}\left(x_{j+1}, v_{j}\right) \leq \frac{1}{(1-\delta)^{2}} \exp \left\{\frac{\delta\left|v_{j}\right|^{2}}{2(1-\delta)}\right\} \mu\left(v_{j}\right) .
$$

Then similar as (4.13), we have, for sufficiently large $T_{0}>0$ and for $k=\hat{C}_{5} T_{0}^{5 / 4}$, that

$$
\int_{\prod_{j=1}^{k-2} \mathcal{V}_{j}} \mathbf{1}_{\left\{t_{k-1}>0\right\}} \prod_{j=1}^{k-2} \mu_{\theta}\left(x_{j+1}, v_{j}\right)\left\{n\left(x_{j}\right) \cdot v_{j}\right\} \mathrm{d} v_{j} \leq\left(\frac{1}{2}\right)^{\hat{C}_{6} T_{0}^{5 / 4}},
$$

for some generic constant $\hat{C}_{5}>0$ and $\hat{C}_{6}>0$. Then by (6.17) and (6.18), we have

$$
\begin{aligned}
F^{n+1} & \geq-C \mu_{\theta}\left(x_{1}, v\right)\left\{1+\left\|h_{0}\right\|_{L^{\infty}}\right\} \int_{\prod_{j=1}^{k-2} \mathcal{V}_{j}} \mathbf{1}_{\left\{t_{k-1}>0\right\}} \prod_{j=1}^{k-2} \mu_{\theta}\left(x_{j+1}, v_{j}\right)\left\{n\left(x_{j}\right) \cdot v_{j}\right\} \mathrm{d} v_{j} \\
& \geq-C \mu_{\theta}\left(x_{1}, v\right)\left\{1+\left\|h_{0}\right\|_{L^{\infty}}\right\} \cdot\left(\frac{1}{2}\right)^{\hat{C}_{6} T_{0}^{5 / 4}} .
\end{aligned}
$$

Since $T_{0}>0$ can be taken arbitrarily large, we have $F^{n+1} \geq 0$. This then proves (6.12) and (6.13). Finally, with the uniform estimates (6.12) in hand, we can use a similar argument as one in [19, Theorem 4.1] to show that $h^{n}, n=0,1,2 \cdots$, is a Cauchy sequence in $L^{\infty}$. We omit here for brevity. The solution is obtained by taking the limit $n \rightarrow \infty$. If $\Omega$ is convex and the compatibility condition (1.19) holds, the continuity is a direct consequence of the $L^{\infty}$-convergence. The uniqueness is standard. The proof of Proposition 6.2 is complete.

Acknowledgments. Renjun Duan is partially supported by the General Research Fund (Project No. 14302817). Feimin Huang is partially supported by National Center for Mathematics and Interdisciplinary Sciences, AMSS, CAS and NSFC Grant No.11688101. Yong Wang is partly supported by NSFC Grant No. 11401565 and 11688101.

\section{REFERENCES}

[1] K. Aoki, F. Golse and S. Kosuge, The steady Boltzmann and Navier-Stokes equations, Bull. Inst. Math. Acad. Sin. (N.S.) 10 (2015), no. 2, 205-257. 
[2] L. Arkeryd and C. Cercignani, A global existence theorem for the initial-boundary value problem for the Boltzmann equation when the boundaries are not isothermal, Arch. Rational Mech. Anal. 125 (1993), no. 3, 271-287.

[3] L. Arkeryd, C. Cercignani and R. Illner, Measure solutions of the steady Boltzmann equation in a slab, Comm. Math. Phys. 142 (1991), no. 2, 285-296.

[4] L. Arkeryd, R. Esposito, R. Marra, A. Nouri, Stability of the laminar solution of the Boltzmann equation for the Benard problem, Bull. Inst. Math. Acad. Sin. (N.S.) 3 (2008), no. 1, 51-97.

[5] L. Arkeryd and N. Maslova, On diffuse reflection at the boundary for the Boltzmann equation and related equations, J. Statist. Phys. 77 (1994), no. 5-6, 1051-1077.

[6] L. Arkeryd and A. Nouri, Boltzmann asymptotics with diffuse reflection boundary condition, Monatsh. Math. 123 (1997), 285-298.

[7] L. Arkeryd and A. Nouri, $L^{1}$ solutions to the stationary Boltzmann equation in a slab, Ann. Fac. Sci. Toulouse Math. (6)9 (2000), no. 3, 375-413.

[8] L. Arkeryd and A. Nouri, The stationary Boltzmann equation in $\mathbb{R}^{n}$ with given indata, Ann. Scuola Norm. Sup. Pisa Cl. Sci. (5) 1 (2002), no. 2, 359-385.

[9] M. Briant and Y. Guo, Asymptotic stability of the Boltzmann equation with Maxwell boundary conditions, $J$. Diff. Equa. 261 (2016), no. 12, 7000-7079.

[10] R.E. Caflisch, The Boltzmann equation with a soft potential. I. Linear, spatially- homogeneous, Commun. Math. Phys. 74 (1980), 71-95.

[11] R. E. Caflisch, The Boltzmann equation with a soft potential. II. Nonlinear, spatially-periodic, Comm. Math. Phys. 74 (1980), 97-109.

[12] C. Cercignani, On the initial-boundary value problem for the Boltzmann equation, Arch. Rational Mech. Anal. 116 (1992), 307-315.

[13] C. Cercignani, R. Illner, M. Pulvirenti, The Mathematical Theory of Dilute Gases, Springer-Verlag, New York, 1994.

[14] C.-C. Chen, I.-K. Chen, T.-P. Liu, Y. Sone, Thermal transpiration for the linearized Boltzmann equation, Comm. Pure Appl. Math. 60 (2007), no. 2, 147-163.

[15] L. Desvillettes, Convergence to equilibrium in large time for Boltzmann and B.G.K. equations, Arch. Rational Mech. Anal. 110 (1990), no. 1, 73-91.

[16] L. Desvillettes, C. Villani, On the trend to global equilibrium for spatially inhomogeneous kinetic systems: The Boltzmann equation, Inv. Math. 159 (2005), 243-316.

[17] R.J. DiPerna, P.-L. Lions, On the Cauchy problem for Boltzmann equation: Global existence and weak stability, Ann. of Math. 130 (1989), 321-366.

[18] R.J. Duan, F.M. Huang, Y. Wang, T. Yang, Global well-posedness of the Boltzmann equation with large amplitude initial data, Arch. Rational. Mech. Anal. 225 (2017), no. 1, 375-424.

[19] R.J. Duan, Y. Wang, The Boltzmann equation with large-amplitude initial data in bounded domains, arXiv:1703.07978

[20] R.J. Duan, T, Yang, H.J. Zhao, The Vlasov-Poisson-Boltzmann system for soft potentials. Math. Models Methods Appl. Sci. 23 (2013), no. 6, 979-1028.

[21] R. Esposito, Y. Guo, C. Kim, R. Marra, Non-isothermal boundary in the Boltzmann theory and Fourier law, Commun. Math. Phys. 323 (2013), 177-239.

[22] R. Esposito, Y. Guo, C. Kim, R. Marra, Stationary solutions to the Boltzmann equation in the hydrodynamic limit, Ann. PDE 4 (2018), no. 1, Art. 1, 119 pp.

[23] R. Esposito, J.L. Lebowitz and R. Marra, Hydrodynamic limit of the stationary Boltzmann equation in a slab, Comm. Math. Phys. 160 (1994), no. 1, 49-80.

[24] R. Esposito, J.L. Lebowitz and R. Marra, The Navier-Stokes limit of stationary solutions of the nonlinear Boltzmann equation, J. Stat. Phys. 78 (1995), 389-412.

[25] R.T. Glassey, The Cauchy Problem in Kinetic Theory, Society for Industrial and Applied Mathematics (SIAM), Philadelphia, 1996.

[26] J.P. Guiraud, Probleme aux limites intérieur pour l'équation de Boltzmann linéaire, J. de Méc. 9(3) (1970), 183-231.

[27] J.P. Guiraud, Probleme aux limites intérieur pour l'équation de Boltzmann en régime stationnaire, faiblement non linéaire, J. de Méc. 11(2)(1972), 443-490.

[28] Y. Guo, Classical solutions to the Boltzmann equation for molecules with an angular cutoff, Arch. Ration. Mech. Anal. 169 (2003), no. 4, 305-353.

[29] Y. Guo, Decay and continuity of the Boltzmann equation in Bounded domains, Arch. Rational. Mech. Anal. 197 (2010), 713-809.

[30] Y. Guo, C. Kim, D. Tonon, A. Trescases, Regularity of the Boltzmann equation in convex domains, Invent. Math. 207 (2017), no. 1, 115-290.

[31] Y. Guo, S.-Q. Liu, The Boltzmann equation with weakly inhomogeneous data in bounded domain, J. Funct. Anal. 272 (2017), no. 5, 2038-2057. 
[32] K. Hamdache, Initial-boundary value problems for the Boltzmann equation: global existence of weak solutions, Arch. Rational Mech. Anal. 119 (1992), no. 4, 309-353.

[33] C. Kim, D. Lee, The Boltzmann equation with specular boundary condition in convex domains, Comm. Pure Appl. Math. 71 (2018), no. 3, 411-504.

[34] H.-W. Kuo, T.-P. Liu, L.-C. Tsai, Equilibrating effects of boundary and collision in rarefied gases, Comm. Math. Phys. 328 (2014), no. 2, 421-480.

[35] H.-W. Kuo, T.-P. Liu, L.-C. Tsai, Free molecular flow with boundary effect, Comm. Math. Phys. 318 (2013), no. 2, 375-409.

[36] S.-Q. Liu, X.-F. Yang, The initial boundary value problem for the Boltzmann equation with soft potential, Arch. Rational. Mech. Anal. 233 (2017), no. 1, 463-541.

[37] N.B. Maslova, Nonlinear Evolution Equations, Kinetic Approach. World Scientific, Singapore, 1993.

[38] S. Mischler, Kinetic equations with Maxwell boundary conditions, Ann. Sci. Ec. Norm. Super. 43 (2010), no. 5, 719-760.

[39] Y. Sone, Molecular Gas Dynamics: Theory, Techniques, and Applications. Birkhauser, Boston, 2007.

[40] R.M. Strain, Asymptotic stability of the relativistic Boltzmann equation for the soft potentials, Comm. Math. Phys. 300 (2010), no. 2, 529-597.

[41] R.M. Strain, Optimal time decay of the non cut-off Boltzmann equation in the whole space, Kinet. Relat. Models 5 (2012), 583-613.

[42] R.M. Strain, Y. Guo, Almost exponential decay near Maxwellian, Comm. Part. Diff. Eqs. 31 (2006), 417-429.

[43] R.M. Strain, Y. Guo, Exponential decay for soft potentials near Maxwellian, Arch. Rational. Mech. Anal. 187 (2008), 287-339.

[44] S. Ukai and K. Asano, On the Cauchy problem of the Boltzmann equation with a soft potential, Publ. Res. Inst. Math. Sci. 18 (1982), no. 2, 477-519.

[45] I. Vidav, Spectra of perturbed semigroups with applications to transport theory, J. Math. Anal. Appl. 30 (1970), 264-279.

[46] S.-H. Yu, Stochastic formulation for the initial-boundary value problems of the Boltzmann equation, Arch. Ration. Mech. Anal. 192 (2009), no. 2, 217-274.

(R.-J. Duan) Department of Mathematics, The Chinese University of Hong Kong, Hong Kong E-mail address: rjduan@math.cuhk.edu.hk

(F. M. Huang) Institute of Applied Mathematics, Academy of Mathematics and Systems Science, Chinese Academy of Sciences, Beijing 100190, China, and University of Chinese Academy of Sciences E-mail address: fhuang@amt.ac.cn

(Y. Wang) Institute of Applied Mathematics, Academy of Mathematics and Systems Science, Chinese Academy of Sciences, Beijing 100190, China, and University of Chinese Academy of Sciences

E-mail address: yongwang@amss.ac.cn

(Z. Zhang) Department of Mathematics, The Chinese University of Hong Kong, Hong Kong

E-mail address: zzhang@math.cuhk.edu.hk 